UNIVERSIDADE DE SÃO PAULO

FACULDADE DE MEDICINA DE RIBEIRÃO PRETO

PROGRAMA DE PÓS-GRADUAÇÃO EM CIÊNCIAS DA SAÚDE APLICADAS AO APARELHO LOCOMOTOR

\begin{abstract}
AVALIAÇÃO DOS FATORES ASSOCIADOS À EXTRUSÃO MENISCAL NO COMPARTIMENTO FEMOROTIBIAL MEDIAL NA PRESENÇA E NA AUSÊNCIA DA CARGA CORPÓREA
\end{abstract}

EVERALDO GREGIO JUNIOR

Ribeirão Preto 
UNIVERSIDADE DE SÃO PAULO

FACULDADE DE MEDICINA DE RIBEIRÃO PRETO

PROGRAMA DE PÓS-GRADUAÇÃO EM CIÊNCIAS DA SAÚDE APLICADAS

AO APARELHO LOCOMOTOR

\section{AVALIAÇÃO DOS FATORES ASSOCIADOS À EXTRUSÃO MENISCAL NO COMPARTIMENTO FEMOROTIBIAL MEDIAL NA PRESENÇA E NA AUSÊNCIA DA CARGA CORPÓREA}

Tese de Doutorado apresentada a Faculdade de Medicina de Ribeirão Preto da Universidade de São Paulo para obtenção do Título de Doutor em Ciências

Orientado: Everaldo Gregio Junior

Orientador: Prof Dr. Marcello Henrique

Nogueira - Barbosa

Ribeirão Preto 
Autorizo a reprodução e divulgação total ou parcial deste trabalho, por qualquer meio convencional ou eletrônico, para fins de estudo e pesquisa, desde que citada a fonte.

Gregio-Junior, Everaldo

Avaliação dos fatores associados à extrusão meniscal no compartimento femorotibial medial na presença e na ausência da carga corpórea, 2015.

105p. : il. ; $30 \mathrm{~cm}$

Tese de Doutorado, apresentada à Faculdade de Medicina de Ribeirão Preto/USP.

Orientador: Nogueira-Barbosa, Marcello Henrique.

1. Joelho. 2. Ferimentos e Lesões. 3. Imagem por Ressonância Magnética. 4. Ultrassom. 


\section{FOLHA DE APROVAÇÃO}

\section{Everaldo Gregio Junior}

"Avaliação dos fatores associados à extrusão meniscal no compartimento femorotibial medial na presença e na ausência da carga corpórea”

Tese de Doutorado apresentada a Faculdade de Medicina de Ribeirão Preto da Universidade de São

Paulo para obtenção do Título de Doutor em Ciências

Orientadora: Prof. Dr. Marcello Henrique Nogueira-Barbosa

Aprovado em:

\section{Banca Examinadora}

Prof.(a). Dr.(a).

Instituição: Assinatura:

Prof.(a). Dr.(a).

Instituição: Assinatura:

Prof.(a). Dr.(a).

Instituição: Assinatura: 


\section{DEDICATÓRIA}

Dedico esta tese as pessoas mais importantes para mim, minha mãe Maria de Lourdes Marcondes Pereira Gregio, e ao meu pai Everaldo Gregio, responsáveis pela minha vida e a quem devo meu caráter e disciplina.

O amor e apoio incondicional de vocês me permitiram alcançar todos os meus sonhos, e aos 35 anos me considero um homem realizado e pronto para traçar novos objetivos. 


\section{AGRADECIMENTO ESPECIAL}

Ao meu amigo, professor e orientador Professor Doutor Marcello Henrique Nogueira-Barbosa que ao me acolher como seu residente abriu as portas para a realização dos meus sonhos acadêmicos. Com paciência e tranqüilidade me transmitiu um pouco de seus conhecimentos, me ensinando principalmente a apreender.

Contudo, foi com a convivência que tive a oportunidade de observar sua postura e condutas profissionais e pessoais dia a dia, que se tornaram meus exemplos para a busca do sucesso.

Obrigado. 


\section{AGRADECIMENTOS}

Ao Michel Daoud Crema e ao Professor Doutor Marcello Henrique NogueiraBarbosa por participarem ativamente desse projeto, do inicio até sua publicação. Mas principalmente por confiarem na minha capacidade como professor, que após várias aulas ministradas em congressos, tornaram esses anos de pós graduação os mais importantes e satisfatórios da minha vida profissional.

Ao amigo da pós graduação Rafael Reis que contribuiu diretamente para a realização deste projeto, de forma fundamental, se mostrou o melhor estatístico.

Aos colegas de residência de Radiologia, pelo companheirismo e compreensão, destacando-se Francisco Abaeté das Chagas-Neto, Ricardo Noda Navarro, Sandra Nakamura, Mariana Basso Polezzi e Vitor Faeda Dalto, graças a vocês me senti em casa no HC.

À Rita Cossalter secretaria do Programa de Pós Graduação em Ciências da Saúde Aplicadas ao Aparelho Locomotor, que do início ao fim me socorreu e tirou todas as minhas dúvidas.

Aos amigos da ressonância magnética, biomédicos, técnicos, enfermeiros e auxiliares, pela atenção especial com a minha pesquisa ao longo de doze meses, longos fins de semana, de muitas conversas e risadas.

À Coordenação de Aperfeiçoamento de Pessoal de Nível Superior (CAPES) pela bolsa de estudos de Doutorado. 


\section{EPÍGRAFE}

Posso ter defeitos, viver ansioso e ficar irritado algumas vezes, mas não esqueço de que a minha vida é a maior empresa do mundo.

E que posso evitar que ela vá à falência. Ser feliz é reconhecer que vale a pena viver, apesar de todos os desafios, incompreensões e períodos de crise.

Ser feliz é deixar de ser vítima dos problemas e se tornar um autor da própria história.

É atravessar desertos fora de si, mas ser capaz de encontrar um oásis no recôndito da sua alma.

É agradecer a Deus a cada manhã pelo milagre da vida.

Ser feliz é não ter medo dos próprios sentimentos. É saber falar de si mesmo. É ter coragem para ouvir um "não".

É ter segurança para receber uma crítica, mesmo que injusta...

Pedras no caminho ? Guardo todas, um dia vou construir um castelo...

Fernando Pessoa 


\section{LISTA DE FIGURAS}

Figura 1. Representação gráfica das regiões dos meniscos do joelho. .25

Figura 2. Representação gráfica de uma lesão meniscal do tipo radial. .26

Figura3. Representação gráfica de uma lesão meniscal do tipo horizontal .27

Figura 4. Representação gráfica de uma lesão meniscal do tipo vertical .28

Figura 5. Representação gráfica de uma lesão meniscal do tipo Flap .29

Figura 6. Representação gráfica de uma lesão meniscal do tipo maceração .30

Figura 7. Representação gráfica de uma lesão meniscal do tipo complexa .30

Figura 8. Representação gráfica de uma lesão meniscal do tipo alça de balde. .31

Figura 9. Posicionamento de um participante masculino de 28 anos .43

Figura 10. RM do joelho de um participante masculino de 43 anos .46

Figura 11. Radiografia realizada de ambos os joelhos em incidência anteroposterior em ortostase com carga e apoio bipodal

Figura 12. Pontos de referência de medida do joelho 49

Figura 13. Grau 0 51

Figura 14. Grau 1 52

Figura 15. Grau 2 53

Figura 16. Mensuração da extrusão meniscal medial (em $\mathrm{mm}$ ) de acordo com a presença ou ausência de lesão .60 
Figura 17. Distribuição das medidas de extrusão meniscal medial de acordo com a presença ou ausência de lesão .62

Figura 18. Graduação da extrusão meniscal medial (em $\mathrm{mm}$ ) de acordo com a presença ou ausência de dor .64

Figura 19. Mensuração da extrusão meniscal medial (em $\mathrm{mm}$ ) de acordo com a presença ou ausência de artrose radiográfica .65 


\section{LISTA DE TABELAS}

Tabela 1. Critérios radiográficos para classificação de osteoartrite .47

Tabela 2. Comparação das graduações de extrusão meniscal medial entre ultrassom e ressonância magnética (RM) para o mesmo examinador. .56

Tabela 3. Análise interobservador para avaliação de extrusão meniscal medial em cada método de imagem.

Tabela 4. Análise intraobservador para avaliação de extrusão meniscal medial em cada método de imagem.

Tabela 5. Desempenho diagnóstico do ultrassom na detecção da extrusão meniscal medial comparada com a ressonância magnética

Tabela 6. Frequência da distribuição dos graus de extrusão meniscal de acordo com a presença ou ausência de lesão

Tabela 7. Extrusão meniscal medial (em $\mathrm{mm}$ ) de acordo com o tipo de lesão encontrada 
LISTA DE ABREVIATURAS

$\mathrm{OA}$

Osteoartrite

$\mathrm{RM}$

Ressonância Magnética

US

Ultrassonografia

IMC

Índice de Massa Corpórea

KL

Classificação de Kellgrenn \& Lawrence

$\mathrm{RP}$

Raiz posterior

LCM

Ligamento colateral medial

FOV

Field of View

WOMAC

Western Ontário e McMaster

EVA

Escala Visual Analógica

$\mathrm{mm}$

Milímetros

$\mathrm{ms}$

Milissegundos

$\mathrm{kg} / \mathrm{m}^{2}$

Quilograma por metro quadrado

$\mathrm{MHz}$

TSE

Turbo Spin-Echo

ETL

NEX

Número de excitações 
TR

Tempo de Repetição

$\mathrm{T}$

Tesla

$\mathrm{Hz}$

Hertz

$\mathrm{cm}$

Centímetro

$\kappa$

Índice Kappa

CIC

Correlação Intra Classe

IC

Intervalo de Confiança

CR

Computed radiography 


\section{RESUMO}

Introdução: As alterações meniscais causadas por lesões degenerativas ou traumáticas podem levar a importantes alterações na estrutura dos mesmos. Pouco se sabe sobre o efeito direto da carga corpórea nos compartimentos femorotibiais sobre os diferentes graus de extrusão meniscal. Objetivos: Avaliar a extrusão meniscal no compartimento femorotibial medial, nas posições em decúbito e ortostática, por meio da ultrassonografia (US). Avaliar o desempenho da US na avaliação meniscal na posição em decúbito, usando a ressonância magnética (RM) como padrão de referência. Material e Métodos: 104 indivíduos com dor crônica no joelho realizaram exames de ultrassonografia e ressonância magnética. Dois radiologistas avaliaram a extrusão meniscal por US e RM e a graduaram:0 $(<2 \mathrm{~mm}), 1(\geq 2 \mathrm{~mm}$ and $<4 \mathrm{~mm})$, and $2(\geq 4$ mm).Um avaliador realizou a mensuração da extrusão meniscal dos indivíduos nas posições em decúbito dorsal e ortostática, e para avaliar os efeitos dessas medidas foi utilizada a correlação intraclasse (CIC) e teste pareado de Wilcoxon. Para comparar os resultados obtidos pela mensuração entre grupos com presença e ausência de lesão foi utilizado o teste $\mathrm{T}$ não pareado, com $\mathrm{p}<0,05$ considerado como significativo. Resultados: Houve concordância substancial entre os examinadores quando comparadas a avaliação entre US e RM (CIC: 0,73 e 0,70). O US mostrou excelente sensibilidade (95\% e $96 \%$, cada examinador) e boa especificidade ( $82 \%$ e $70 \%$, cada examinador). Houve diferença significativa dos valores médios de extrusão meniscal entre as posições em decúbito e ortostase $(\mathrm{p}=0,0002)$. Todos os meniscos com extrusão $\geq 3 \mathrm{~mm}$ pela US apresentavam lesão meniscal confirmada pela RM ( $\mathrm{p}<0,0001)$. Conclusão: A avaliação de extrusão meniscal via US mostra excelente desempenho diagnóstico. Há variação na extrusão do menisco medial entre as posições decúbito e ortostase, com maior extrusão na posição ereta. 
Palavras-chave: joelho, extrusão meniscal, ultrassonografia, imagem por ressonância magnética 


\begin{abstract}
Background: Meniscal abnormalities related to degenerative or traumatic lesions may lead to significant changes in meniscal structure. There is lack knowledge about the direct effect of weight bearing in femorotibial compartments on the diverse meniscal extrusion levels. Objectives: To evaluate meniscal extrusion on medial femorotibialcompartmentby ultrasonography (US), both in dorsal decubitus and orthostatic positions. To evaluate US performance inassessment of meniscus in dorsal decubitus, using magnetic resonance (MR) as a standard method. Material andMethods: A total of 104 subjects with chronic pain on the knee underwent US and MR. Meniscal extrusion was assessed by two radiologists using US and RM, being graded as: $0(<2 \mathrm{~mm}), 1(\geq 2 \mathrm{~mm}$ and $<4 \mathrm{~mm})$, and $2(\geq 4 \mathrm{~mm})$. One radiologist performed measurements of meniscal extrusion of the participants in dorsal decubitus and orthostatic positions. Intra-class coefficient (ICC) and Wilcoxon paired t-test were used to analyze the effects of such measurements. To compare data from groups with and without lesions we used non-paired t-test was used, with values of $\mathrm{p}<$ 0,05considered to be significant. Results: There was substantial agreement between readers when US and MR evaluations were compared (ICC: 0,73 e 0,70). US showed excellent sensitivity (95\% e 96\%, each reader) and good specificity (82\% e 70\%, each reader). There was significant difference onmedium values of meniscal extrusion regarding dorsal decubitus and orthostatic positions $(\mathrm{p}=0,0002)$. All subjects that presented meniscal extrusion $\geq 3 \mathrm{~mm}$ on US had meniscal lesion confirmed by RM ( $\mathrm{p}<$ 0,0001). Conclusion: US has an excellent diagnostic performance in assessment of meniscal extrusion. Meniscal extrusion varies from dorsal decubitus and orthostatic positions, being higher while in upright position.
\end{abstract}


Keywords: knee, meniscal extrusion, ultrasonography, magnetic resonance imaging 


\section{SUMÁRIO}

1. INTRODUÇÃO ............................................................................................................23

1. Estrutura e Função dos Meniscos do Joelho......................................................................24

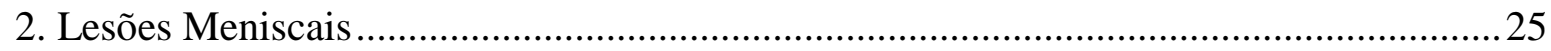

3. Alterações Meniscais relacionadas à osteoartrite ……………………………………....... 32

2. JUSTIFICATIVA ..............................................................................................................36

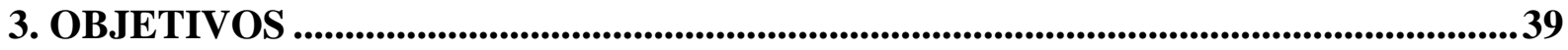

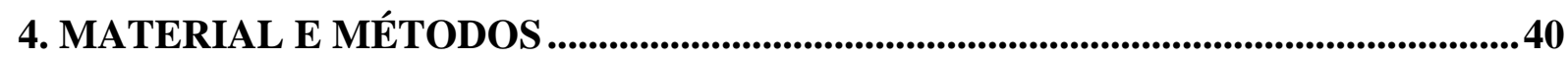

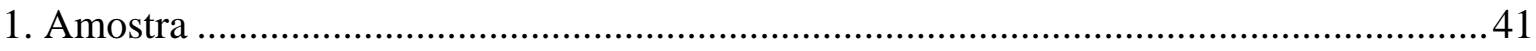

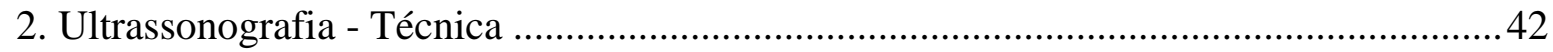

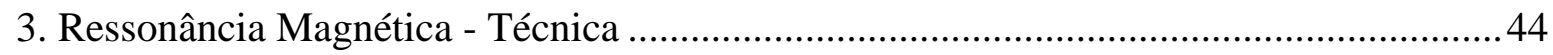

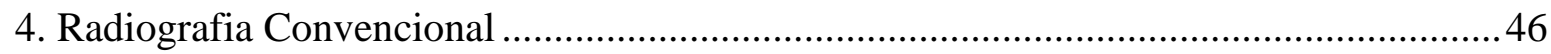

5. Ressonância Magnética e Ultrassonografia - Interpretação............................................ 48

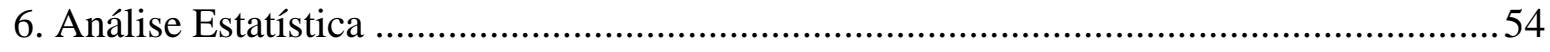

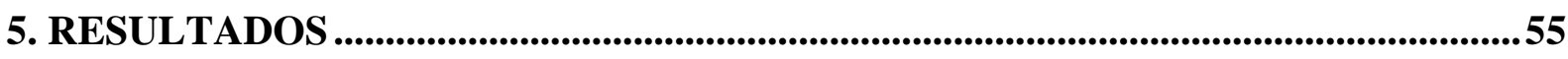

1. Validação da avaliação de extrusão meniscal medial por US e RM .................................56

2. Mensuração de extrusão meniscal medial com e sem carga por meio do US ..................59

3. Avaliação dos fatores associados à extrusão meniscal medial .......................................... 62

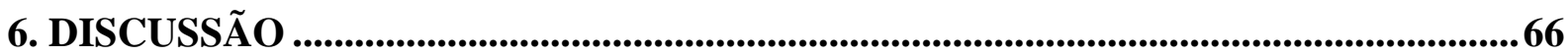

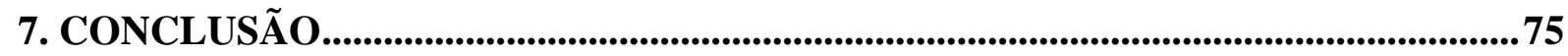

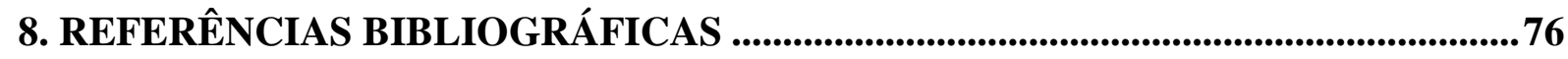

ANEXO A: Carta de Aprovação do Comitê de Ética e Pesquisa............................................ 84 
ANEXO B: Termo de Consentimento Livre e Esclarecido

ANEXO C: Índice WOMAC para Osteoartrose.

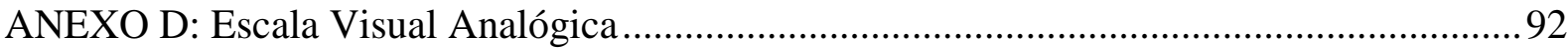

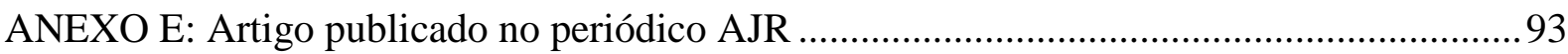

ANEXO F: Trabalho premiado no congresso internacional RSNA 2012 _...........................98

ANEXO G: Trabalho publicado nos anais o RSNA 2012 …................................................99

ANEXO H: Trabalho publicado nos anais do ECR 2013 ................................................. 100

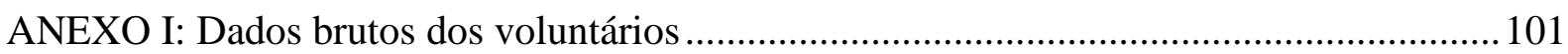




\section{INTRODUÇÃO}




\subsection{Estrutura e Função dos Meniscos do Joelho}

Os meniscos atuam na biomecânica das articulações femorotibiais como absorventes de choque, distribuidores de carga, além de lubrificar e estabilizar estes compartimentos de forma secundária (Seedhonet al.; 1974; Crema et al., 2010). O menisco é uma estrutura fibrocartilaginosa semilunar, triangular, em formato de $\mathrm{C}$ cuja superfície côncava é voltada para a região central do joelho, em conformidade à morfologia do côndilo femoral, e a base de aspecto plano ligada à superfície condilar da tíbia através dos ligamentos anterior e posterior da raiz (Renstrom \& Johnson, 1990). A configuração em três camadas distintas de colágeno dentro do menisco, e a orientação dos feixes de colágeno ao longo dos eixos longo e curto dos meniscos permitem eficiente transmissão de carga e absorção de choques. As fibras longitudinais são orientadas circunferencialmente, resultando na habilidade dos meniscos distribuírem cargas axiais. As fibras radiais são mais frouxas e ajudam a formar uma estrutura para manter os feixes longitudinais juntos e resistir às forças que predispõem à ruptura longitudinal do menisco (Rosas, 2014; Petersen \&Tillman, 1998).

Os meniscos são basicamente divididos em três regiões: corno anterior, corpo e corno posterior (Figura 1), porém os meniscos medial e lateral apresentam morfologias diferentes. $\mathrm{O}$ menisco medial é maior e possui abertura em $\mathrm{C}$ mais ampla, o que promove um aumento da largura anteroposterior, resultando em um corno posterior maior que o anterior. Perifericamente, os meniscos são ancorados à cápsula fibrosa, com o menisco medial firmemente ligado às fibras profundas do ligamento colateral medial, o que limita a sua mobilidade e, presumivelmente, aumentando sua susceptibilidade a lesões quando comparado com o menisco lateral (Rosas, 2014; Rach \& Richmond, 2000). 


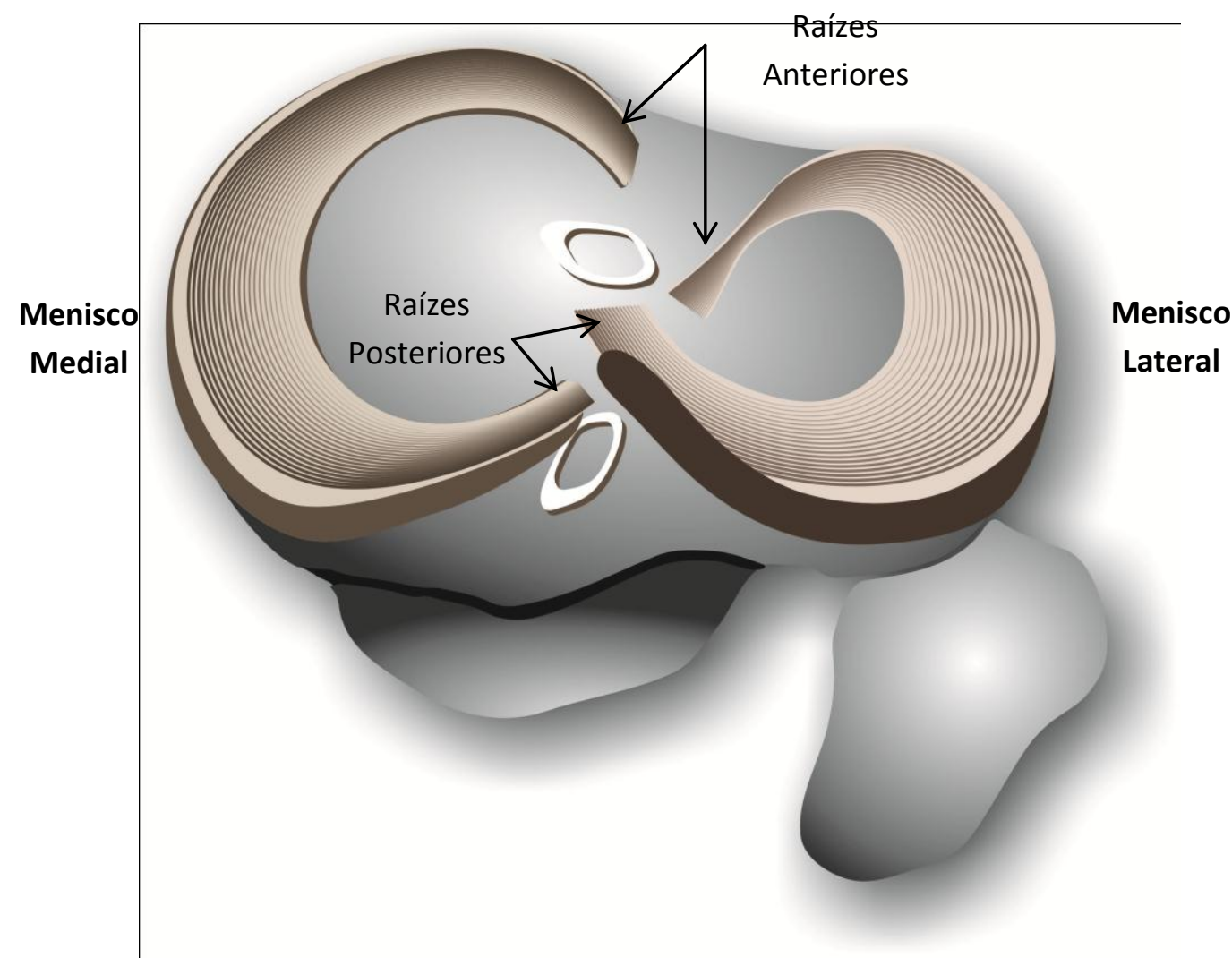

Figura 1. Representação gráfica das regiões dos meniscos medial e lateral do joelho: corpos e cornos anteriores e posteriores (setas).

\subsection{Lesões Meniscais}

As alterações meniscais causadas por lesões degenerativas ou traumáticas podem levar a importantes alterações na microestrutura dos meniscos, podendo reduzir a capacidade de resistir às forças axiais que lhe são aplicadas quando alguma carga é transmitida aos compartimentos femorotibiais, gerando instabilidade e variados graus de extrusão meniscal (Hunter et al., 2006; Wang et al., 2010; Lo et al., 2008).

A maioria das rupturas envolve o corno posterior do menisco, sendo mais frequentes no menisco medial (De Smet, 2002). Rupturas isoladas do corno anterior dos meniscos são incomuns e devem ser diagnosticadas com cautela, porque elas compreendem apenas $2 \%$ das lesões do menisco medial, e $16 \%$ das lesões do menisco lateral (De Smet, 1993). 
São fundamentais não somente o diagnóstico da ruptura meniscal, mas também a descrição morfológica precisa, a fim de orientar as opções de tratamento e avaliar medidas de resultados a longo prazo. Dentre as lesões, destacam-se três tipos básicos: horizontal, vertical, e radial; e as demais lesões configurado subtipos combinados, que são: lesão em flap, lesão radial de raiz posterior (Radial RP), maceração, complexa e "alça de balde" (Harper et al., 2005). Segundo a revisão sistemática de Rosas et al. (2014), elas apresentam as seguintes características:

- A lesão radial envolve o eixo curto e a borda livre do menisco, mas, em contraste com uma ruptura horizontal, segue um percurso perpendicular ao platô tibial e eixo longo do menisco, dividindo o menisco em porções anterior e posterior (Figura 2). Uma lesão de raiz é uma lesão radial localizada na raiz meniscal (anterior ou posterior).

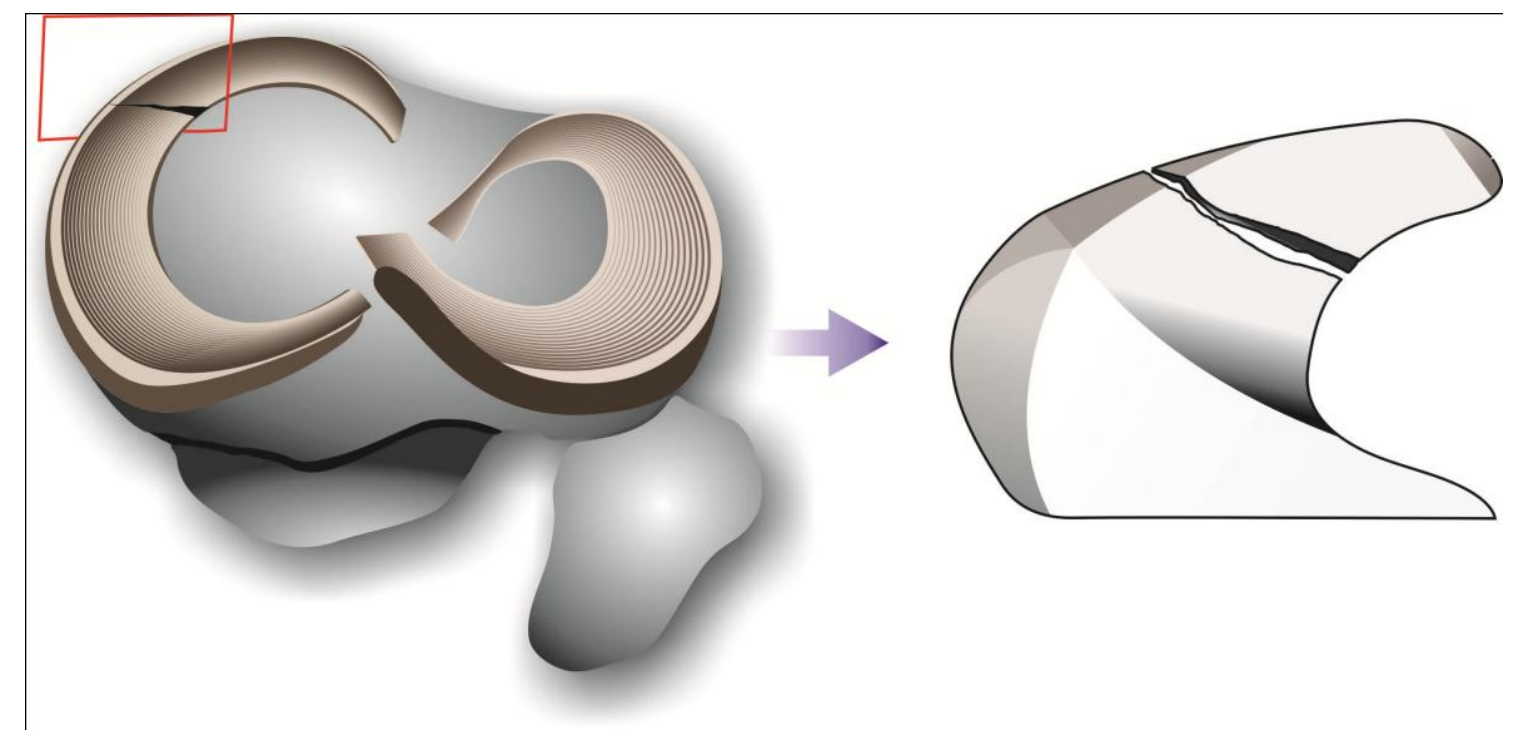

Figura 2. Representação gráfica de uma lesão meniscal do tipo radial. À esquerda visão panorâmica, e à direita visão ampliada em secção transversa do menisco. 
- A lesão horizontal envolve classicamente a borda livre ou uma das superfícies articulares e propaga-se perifericamente, separando assim o menisco em metades superior e inferior (Figura 3).

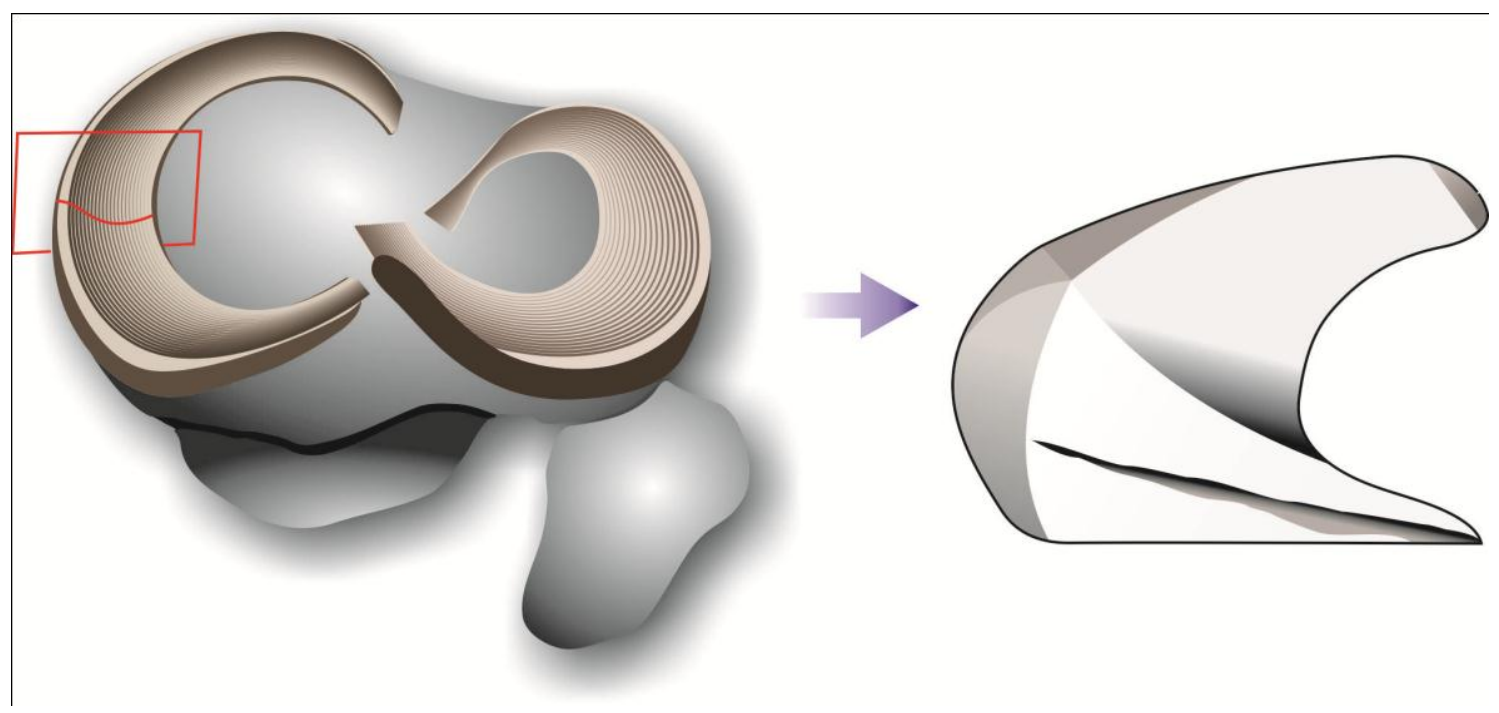

Figura 3. Representação gráfica de uma lesão meniscal do tipo horizontal. À esquerda visão panorâmica, e à direita visão ampliada em secção transversa do menisco.

- A lesão vertical cursa paralela ao eixo longitudinal do menisco e perpendicular ao platô tibial, podendo envolver uma única superfície articular ou ambas as superfícies articulares, separando o menisco em segmentos interior e exterior (Figura 4). 


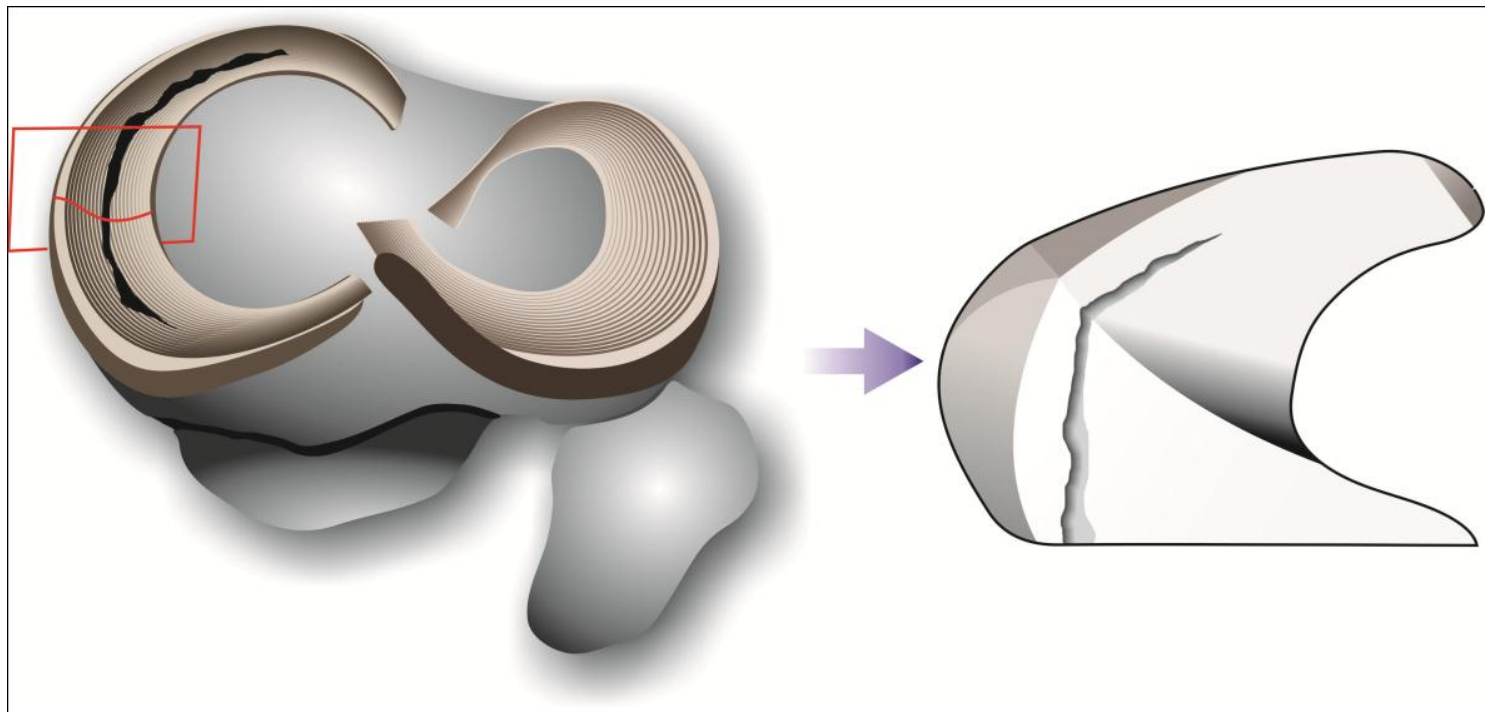

Figura 4. Representação gráfica de uma lesão meniscal do tipo vertical. À esquerda visão panorâmica, e à direita visão ampliada em secção transversa do menisco.

- A lesão do tipo Flap (vertical e horizontal) são rupturas instáveis, pois uma porção do segmento meniscal pode se deslocar. As manifestações clínicas incluem obstrução mecânica sob a forma de bloqueio ou dor. Um Flap vertical, também conhecido como uma ruptura "bico de papagaio", possui componentes tanto longitudinais quanto radiais (Figura 2). Flaps horizontais envolvem um pequeno segmento do menisco que se descola da lâmina meniscal (Figura 5). 


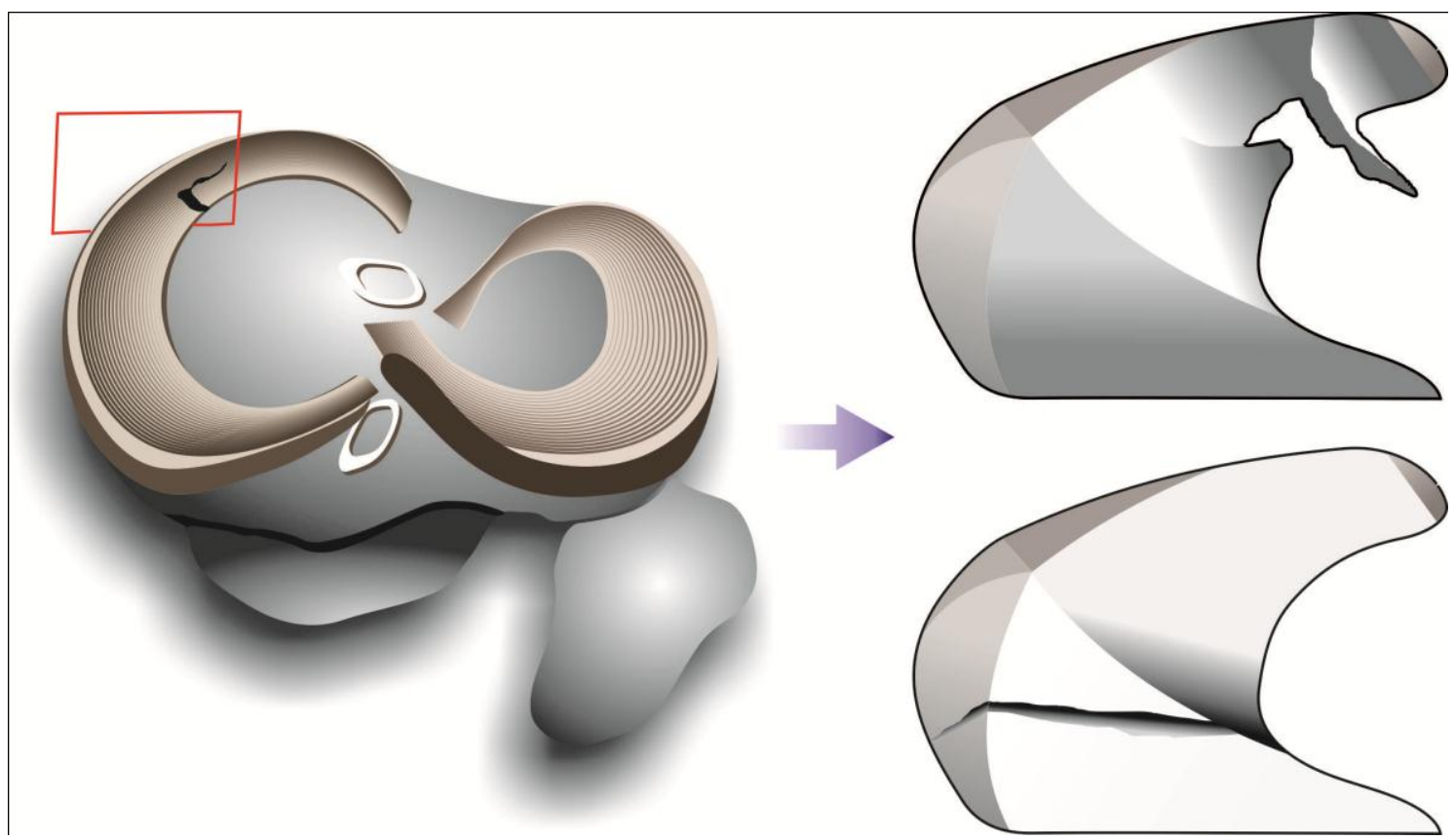

Figura 5. Representação gráfica de uma lesão meniscal do tipo Flap. À esquerda visão panorâmica, e à direita visão ampliada em secção transversa do menisco.

- A lesão por maceração é definida pela perda geral do aspecto morfológico normal com irregularidade e aumento de sinal difuso no tecido meniscal (Figura $6)$. 


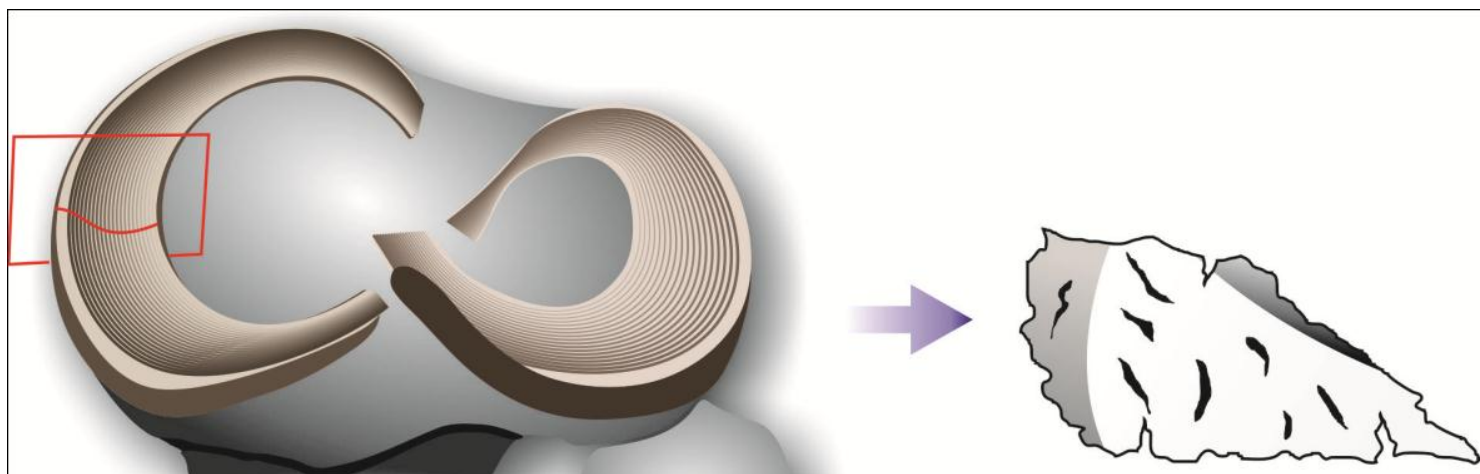

Figura 6. Representação gráfica de uma lesão meniscal do tipo maceração. À esquerda visão panorâmica, e à direita visão ampliada em secção transversa do menisco.

- Lesões que não se enquadram em uma única categoria dentre as anteriores, com orientação da ruptura em vários planos devem ser descritas como complexas. Tipicamente é uma combinação de lesões radiais, horizontais, ou longitudinais, o menisco aparece fragmentado nas imagens (Figura 7).
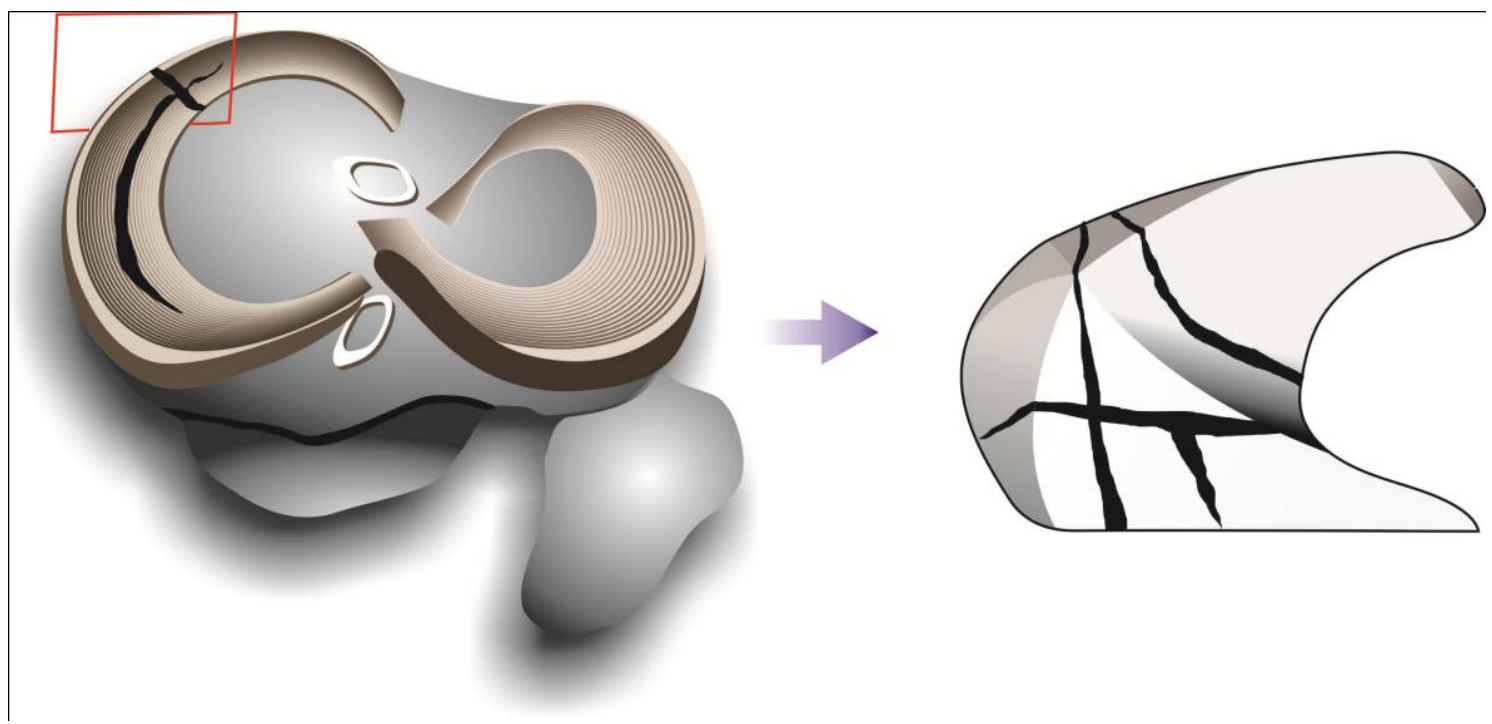

Figura 7. Representação gráfica de uma lesão meniscal do tipo complexa. 
- Lesões em "Alça de Balde" são rupturas verticais longitudinais. O fragmento inferior deslocado se assemelha à alça de um balde. Estas lesões são responsáveis por cerca de $10 \%$ de todas as lesões meniscais (Figura 8).

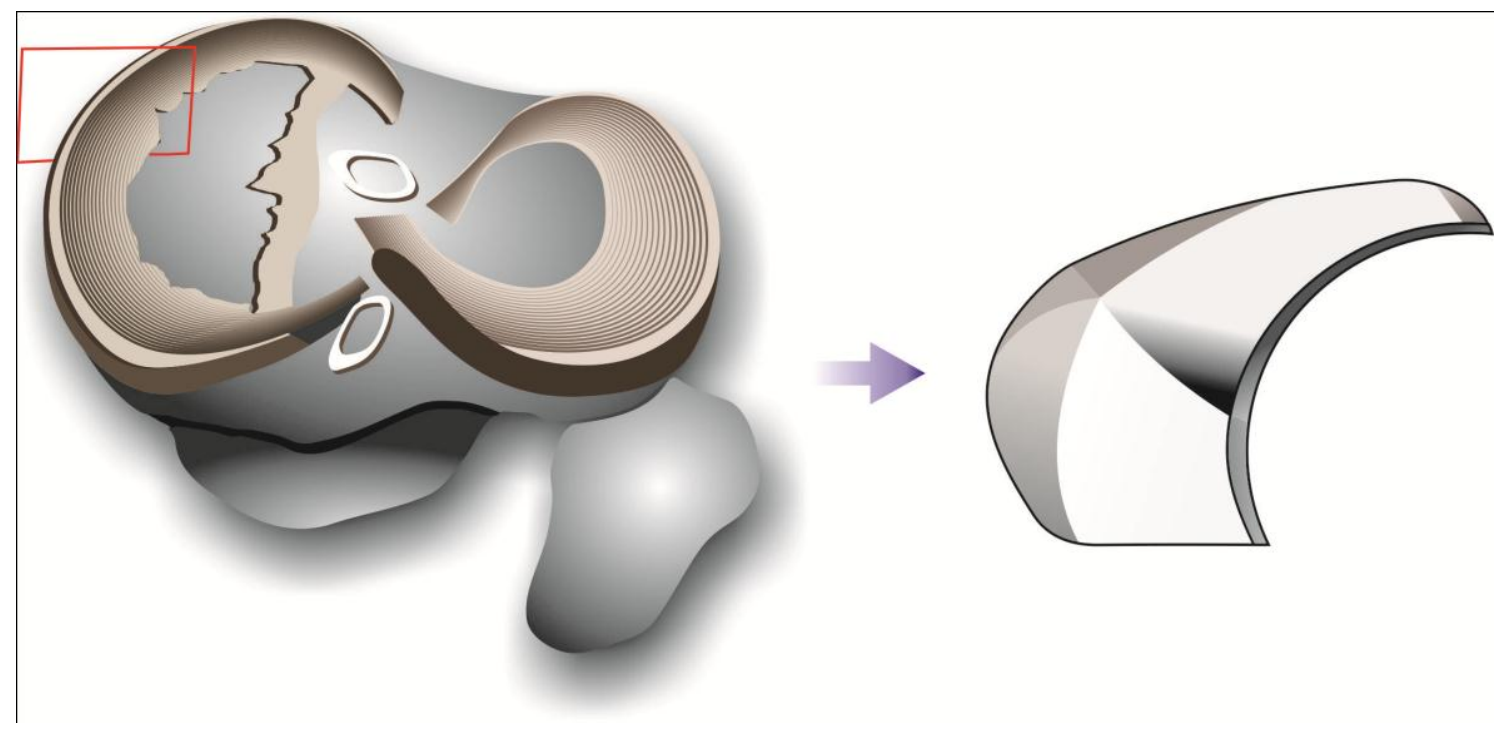

Figura 8. Representação gráfica de uma lesão meniscal do tipo alça de balde. 
As lesões meniscais representam um risco potencial para o desenvolvimento de osteoartrite (OA) femorotibial radiográfica (Englundet al., 2003),. Lesões verticais geralmente ocorrem em associação com traumatismo do joelho, enquanto as lesões horizontais ocorrem em pacientes de meia idade ou mais velhos, resultantes de alterações degenerativas dos meniscos. Recentemente, Kummet al. (2015), mostraram em um estudo longitudinal com adultos de meia idade, sem achados de osteoartrite do joelho, que a presença de uma imagem linear de hipersinal intrameniscal encontrada no compartimento medial em um primeiro exame de $\mathrm{RM}$ realizado,mostrou forte correlação com ruptura meniscal na mesma topografia nos exames seguintes. Sendo assim, sugere-se que uma lesão degenerativa do menisco pode ser o primeiro sinal que anuncia uma doença osteoartrítica na articulação do joelho (Englundet al., 2003).

\subsection{Alterações meniscais relacionadas à Osteoartrite}

A osteoartrite está entre as dez principais doenças não fatais de acordo com a Organização Mundial da Saúde, sendo o joelho uma das articulações mais acometidas. Com o aumento da expectativa de vida aliada ao envelhecimento da população é esperado que a OA se torne à quarta principal causa de incapacidade no ano de 2020 (Woolf \& Pfleger, 2003). Dessa forma, a possibilidade de identificar e diagnosticar precocemente as alterações articulares do joelho associadas ao desenvolvimento da OA, tem motivado inúmeros estudos internacionais nas ultimas três décadas, na busca de resultados que auxiliem seu tratamento e principalmente sua prevenção, reduzindo o impacto negativo na saúde e na qualidade de vida dos pacientes, bem como os custos diretos e indiretos aos sistemas de saúde (Lopez et al., 2006; Zhang et al., 2008 ).

Em 1957, Kellgren \& Lawrence (KL), utilizando radiografias convencionais, aperfeiçoaram seus estudos anteriores definindo a descrição e graduação das alterações 
osteoarticulares encontradas nas diversas fases da OA. Em relação ao joelho, a presença de osteófito marginal inquestionável (KL grau $\geq 2$ ) foi considerada pelos autores um marcador definitivo da OA. Com a evolução dos métodos diagnósticos empregados no estudo do joelho, sobretudo com o avanço da RM, outras alterações foram relacionadas à evolução da patologia (Englundet al., 2008).

Breitenseheret al. (1997) concluíram que a extrusão do menisco medial era um achado frequente nos pacientes com dor no joelho submetidos a RM, porem na época consideraram que tais alterações deveriam ser encaradas como achado secundário indicativo de outras patologias da articulação do joelho, como derrame articular e OA. Mas foi em meados da primeira década dos anos 2000, com o surgimento e maior distribuição de RM de alto campo (entre 1,0 e 2,0 T) e ultra alto campo (> 2,0 T) ao redor do mundo, que o entendimento da fisiopatologia da OA passava realmente a ser explicada pelos métodos de imagem (Lo et al., 2009).

A partir de então,diversos estudos usando RM evidenciaram fatores relacionados à evolução da OA do joelho; dentre elas, as causas mais encontradas foram o derrame articular, as sinovites, as lesões meniscais, e a redução do espaço articular femorotibial com conseqüente perda da cartilagem articular (notadamente acometendo o compartimento femorotibial medial), foram considerados fatores diretamente associados à $\mathrm{OA}$, enquanto que demais características individuais como idade, altos índices de massa corpórea (IMC) e desvios do alinhamento dos membros inferiores em valgo ou varo foram interpretados como fatores indiretos (Breitenseheret al., 1997). Contudo, ainda restavam algumas lacunas do conhecimento sobre o grau de importância de cada uma das alterações acima descritas teriam na evolução e prognóstico da OA do joelho. Em 1948, Fairbank descreveu as alterações degenerativas do joelho encontradas após meniscectomia total. Atualmente sabe-se que, não só a meniscectomia total, mas 
também a meniscectomia parcial são fatores de risco para o desenvolvimento da OA do joelho (Cicuttiniet al, 2002; Englund et al, 2003).

A partir do conhecimento envolvendo as alterações articulares do joelho pósmeniscectomia, trabalhos mais recentes mostraram que mesmo em joelhos sem antecedentes cirúrgicos, a presença de lesão meniscal associada à extrusão mostrou-se isoladamente um poderoso fator de risco para o desenvolvimento de OA radiográfica (Gale et al., 1999; Rennie et al.,2006; Englund, et al.,2009; Crema et al., 2012). Mesmo antes de conhecer esta relação, no final da década de 80 os cistos parameniscais identificados pela ultrassonografia já eram interpretados como achados associados à lesão meniscal (Burket al., 1988; Coral et al., 1989; Peetrons et al., 1990;Seymour et al., 1998).

Com o avanço tecnológico e o conhecimento da importante relação entre a extrusão meniscal e o risco potencial para o desenvolvimento de OA radiográfica, outros métodos de imagem capazes de identificar a extrusão meniscal, como a ultrassonografia, passaram a ter maior aplicação no diagnóstico nas lesões do joelho.

No inicio dos anos 2000, a avaliação do menisco por meio da ultrassonografia já era proposta como parte da rotina do exame de joelho, reforçando características ecográficas normais dos meniscos, como a morfologia triangular ecogênica periférica. Dentre as alterações meniscais descritas pela ultrassonografia, a perda da morfologia triangular ecogênica e a extrusão meniscal foram consideradas como marcadores de lesão (Friedman et al., 2001).

Por se tratar de um método diagnostico dinâmico,a ultrassonografia permite a avaliação dos joelhos com os membros inferiores em diferentes posições, como exemplo sobre a ação da carga corpórea, o que não seria possível de ser realizado com os aparelhos convencionais de RM de forma rotineira. 
Em 2007, Koet al., realizaram um estudo que avaliou a relação da extrusão meniscal na ultrassonografia do joelho sobre a ação da carga corpórea em pacientes com OA radiográfica. Foi mensurado o grau de extrusão meniscal (em milímetros), demonstrando a correlação entre maiores valores de extrusão com OA radiográfica grave. Posteriormente, estes resultados foram reforçados por outros estudos também por meio da ultrassonografia antes e após a ação de carga corpórea (Acebes et al., 2013).

Usando a RM como padrão-ouro, Park et al. (2008) examinaram 41 joelhos por ultrassonografia e RM, exames realizados com intervalo de uma semana e "cegos" quanto aos achados da RM. Os autores apresentaram boa sensibilidade e especificidade da ultrassonografia para detecção das lesões descritas na RM. Contudo, os autores sugerem em seu trabalho que a ultrassonografia não deveria ser usada como método isolado, e sim que, na presença de lesão meniscal ao US, uma RM complementar deveria ser realizada.

Até o momento, nenhum estudo validou as medidas da extrusão meniscal pela ultrassonografia, abrindo o leque de questões a serem respondidas, como: qual a correlação entre os diferentes graus de extrusão meniscal mensurados pela US e os achados da RM? Além disso, a lacuna quanto ao valor clínico das lesões meniscais diagnosticadas de forma isolada pela ultrassonografia, nos faz questionar quais parâmetros ecográficos seriam capazes de confirmar a presença de lesão meniscal propriamente dita. 
JUSTIFICATIVA 


\section{JUSTIFICATIVA}

Pouco se sabe sobre o efeito direto dos diferentes tipos de lesão meniscal detectadas por RM, sobre a extrusão meniscal quando os compartimentos femorotibiais são submetidos à carga corpórea (Stehlinget al., 2012). Outros fatores que elevam potencialmente a carga transmitida aos meniscos, como a elevação do índice de massa corpórea (IMC), alterações no eixo mecânico do joelho, lesões condrais e alterações degenerativas do osso subcondral ainda não foram testados como fatores associados nesse contexto. Esta escassez de dados se deve a dificuldade técnica de se realizar o estudo por RM em ortostase de forma rotineira, onde seria possível estudar a ação da carga corpórea sobre a articulação do joelho simulando uma atitude bipodal habitual. 


\section{OBJETIVOS}

\subsection{Objetivos Primários}

O objetivo geral deste estudo é avaliar a extrusão meniscal e seus variados graus no compartimento femorotibial medial, nas posições em decúbito e ortostática, por meio da ultrassonografia.

\subsection{Objetivos Secundários}

Avaliar o desempenho da ultrassonografia na avaliação da extrusão dos meniscos na posição em decúbito, correlacionando com a ressonância magnética como padrão de referência.

Avaliar as associações dos fatores individuais, como idade, IMC, osteoartrite radiográfica, e as diferentes lesões meniscais, com a variação da extrusão meniscal no compartimento femorotibial medial. 


\section{MATERIAL E MÉTODOS}

Após aprovação pelo comitê de ética em pesquisa do Hospital das Clínicas da Faculdade de Medicina de Ribeirão Preto da USP, Processo HCRP Nº 8989/2011 (Anexo A), foi realizado o recrutamento dos participantes, que concordaram em fazer parte do estudo após explicação individual dos métodos e objetivos da pesquisa e leitura e assinatura do termo de consentimento livre e esclarecido (Anexo B).

Este estudo teve caráter prospectivo, observacional e transversal, utilizando dados clínicos e métodos de diagnostico por imagem, respeitando sempre a mesma ordem: inicio pela ultrassonografia do joelho, seguida da radiografia e por fim a ressonância magnética.

\subsection{Casuística}

Foram incluídos pacientes com dor crônica do joelho, que já possuíam indicação de um exame de ressonância magnética a ser realizada no Hospital das Clínicas da Faculdade de Medicina de Ribeirão Preto da USP. Esses pacientes foram convidados a participar deste estudo, que adicionou um exame de ultrassonografia e uma incidência radiográfica localizada de ambos os joelhos em ortostase, no mesmo dia e local do exame de RM já solicitado (critério de inclusão), garantindo assim maior conforto aos participantes.

Para avaliar a presença ou ausência de dor no joelho estudado, foi utilizado o questionário das universidades de Western Ontário e McMaster (WOMAC) (Anexo C), em conjunto com à escala visual analógica (EVA) (Anexo D), que também avalia a intensidade da dor.

Os exames envolvidos no projeto de pesquisa foram realizados sem ônus ao paciente, sendo agendados em conjunto com exames previamente marcados aos finais 
de semana. O termo de consentimento foi lido e explicado individualmente, ficando a critério de cada sujeito da pesquisa a decisão de participar ou não do estudo.

Os critérios de exclusão constituíram em: cirurgia prévia no joelho avaliado, antecedente de fraturas, doenças inflamatórias em atividade ou neoplasias acometendo esse mesmo joelho, assim como erros na aquisição ou armazenamento das imagens em alguma das etapas da pesquisa.

Do total de 125 pacientes avaliados, 21 foram excluídos pelos seguintes

motivos: sinais de cirurgia prévia (8 casos), fraturas antigas (3 casos), fraturas subcondrais (2 casos), suspeita de lesão neoplásica (1 caso), e perda de dados por erros de aquisição ou armazenamento das imagens ( 7 casos).

O grupo final de pacientes estudados totalizou 104 voluntários (53 homens e 51 mulheres), com média de idade 41,5 \pm 1,8 anos (18 - 70 anos), e IMC médio de 28,7 \pm $5,8 \mathrm{~kg} / \mathrm{m}^{2}\left(20-47 \mathrm{~kg} / \mathrm{m}^{2}\right)$.

\subsection{Ultrassonografia - Técnica}

A ultrassonografia dos participantes foi realizada na sala ao lado da ressonância magnética com aparelho portátil "Logic E” (General Electric Company) sendo utilizado um transdutor linear de alta resolução multifrequencial de 7-12 MHz, associado ao software de cruzamento de feixes, usado para obter melhor resolução superficial de imagem, denominado de "feixe X" pelo fabricante deste modelo de equipamento.

Inicialmente, o paciente foi colocado em decúbito dorsal com ambos os membros inferiores em extensão, semelhante ao posicionamento da RM. Um radiologista com especialização em imagem musculoesquelética e 6 anos de experiência em ultrassonografia musculoesquelética (Leitor 1) foi o responsável por adquirir todas as imagens. 
O corpo do menisco medial foi identificado no corte longitudinal (eixo vertical) no qual era possível identificar o ligamento colateral medial (LCM). Quando o LCM é identificado, o ponto de maior extrusão meniscal no compartimento medial foi sinalizado com dois marcadores na pele (cápsula medicamentosa, Advil@); e na ausência de extrusão meniscal a documentação foi realizada no mesmo eixo, porém usando como referencia a inserção superior do ligamento colateral medial (Figura 9A a C).
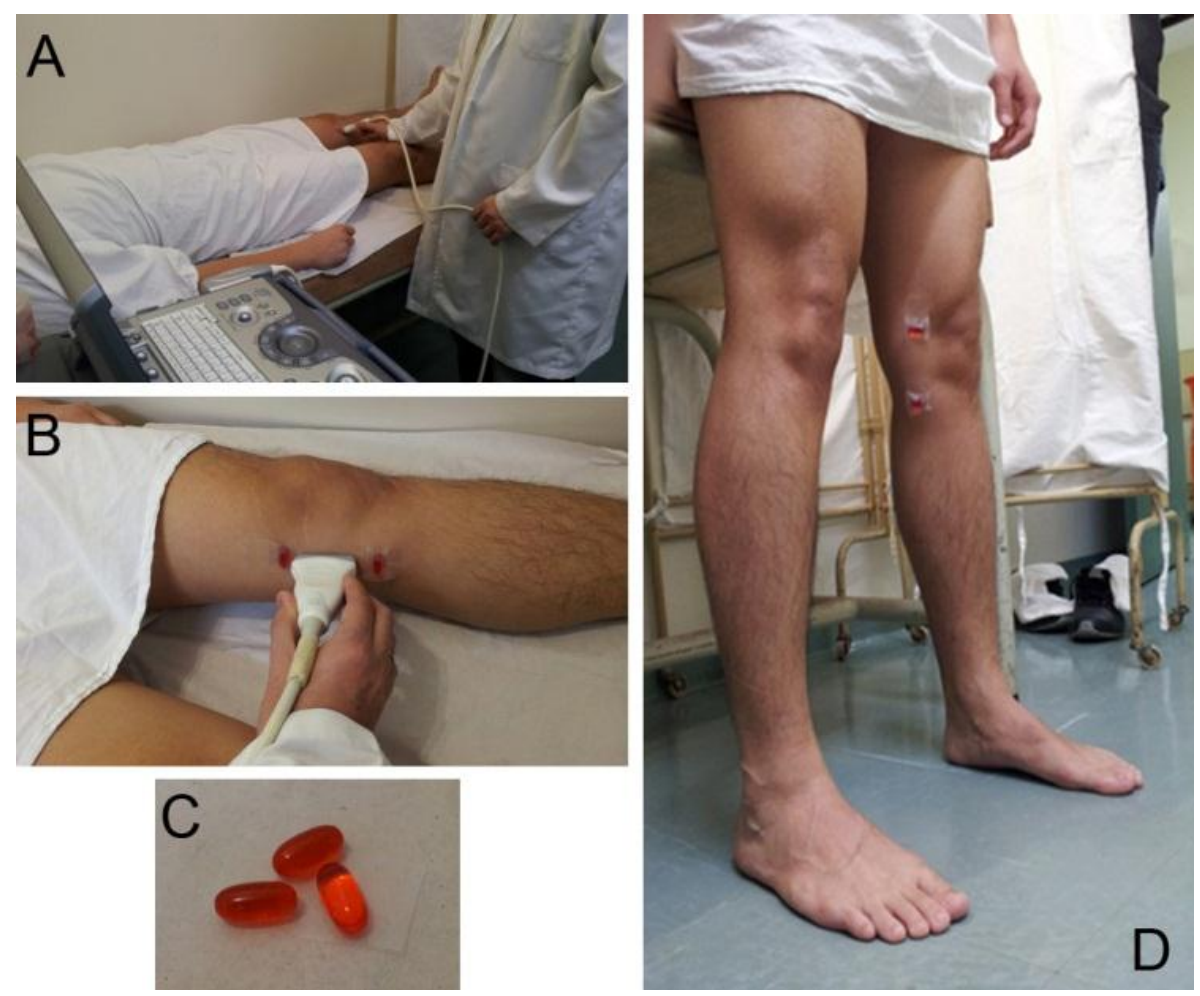

Figura 9. Posicionamento de um participante masculino de 28 anos. A) Aparelho de ultrassonografia portátil "Logic E", utilizado com transdutor linear de 7-12 MHz. B) Posição da avaliação da extrusão meniscal medial adquirida em decúbito dorsal, com os membros inferiores em extensão. Note a presença de dois marcadores na pele (C), no plano de mensuração e captura das imagens ultrassonográficas. D) Posição da avaliação da extrusão meniscal medial adquirida em ortostase, com apoio bipodal e membros inferiores em extensão. 
Depois da realização do exame em decúbito, a presença de extrusão meniscal foi reavaliada em ortostase após o paciente permanecer por 1 minuto sobre apoio bipodal, posicionando o transdutor longitudinalmente na mesma topografia das marcações dérmicas respeitando os contornos ósseos como uma segunda referência (extrusão avaliada exatamente no mesmo plano comparado àquela avaliada em decúbito) (Figura 9D). Ao término do exame ultrassonográfico o paciente foi encaminhado diretamente para a RM portando os marcadores, assim assegurando o mesmo local para as mensurações da US e na RM.

As imagens ultrassonográficas adquiridas pelo examinador (Leitor 1), foram salvas e armazenadas antes das medidas serem realizadas, tanto na avaliação em decúbito como em ortostase, para que um segundo examinador (Leitor 2) com 11 anos de experiência em ultrassonografia musculoesquelética pudesse avaliar posteriormente de forma randomizada e independente.

\subsection{Ressonância Magnética - Técnica}

Todos os participantes foram avaliados (um joelho por participante) com o mesmo aparelho de ressonância magnética de 1.5-T (Achieva, Philips Medical Systems, Best, the Netherlands) usando uma bobina dedicada de joelho (8 - Channel SENSE KneeCoil) com os pacientes em decúbito e sem carga aplicada aos joelhos. O protocolo "convencional" da RM incluiu as sequências obtidas na rotina clínica do serviço, com quatro sequências:

Primeira: Sequência sagital com ponderação intermediária - Turbo SpinEcho,tempo de repetição (TR) 2342 ms, tempo de eco (TE) 50 ms, 224 x 176 matriz, 16 x $16 \mathrm{~cm}$ de campo de visão (FOV), espessura de corte: $4 \mathrm{~mm}$, número de excitações: 4 
(NEX), comprimento do trem de ecos (ETL) 14, largura de banda: $395 \mathrm{~Hz} /$ pixel, Tempo de Aquisição: 2 minutos e 43 segundos;

Segunda: Sequência coronal com ponderação intermediária - Turbo Spin Echo, tempo de repetição (TR) 2342 ms, tempo de eco (TE) 50 ms, 224 x 176 matriz, 16 x 16 cm de campo de visão (FOV), espessura de corte: $4 \mathrm{~mm}$, número de excitações: 4 (NEX), comprimento do trem de ecos (ETL) 14, largura de banda: $386 \mathrm{~Hz} /$ pixel, Tempo de Aquisição: 2 minutos e 30 segundos;

Terceira: Sequência axial com ponderação intermediária - Turbo Spin Echo, tempo de repetição (TR) 3045 ms, tempo de eco (TE) 50 ms, 224 x 176 matriz, 16 x 16 cm de campo de visão (FOV), espessura de corte: $4 \mathrm{~mm}$, número de excitações: 4 (NEX), comprimento do trem de ecos (ETL) 14, largura de banda: $429 \mathrm{~Hz} /$ pixel, Tempo de Aquisição: 3 minutos;

Quarta: Sequência sagital ponderada em T1 - Spin-Echo, tempo de repetição (TR) $532 \mathrm{~ms}$, tempo de eco (TE) $20 \mathrm{~ms}, 292$ x 196 matriz, 20 x $20 \mathrm{~cm}$ de campo de visão (FOV), espessura de corte: $4 \mathrm{~mm}$, número de excitações: 3 (NEX), comprimento do trem de ecos (ETL) 6, largura de banda: $349.5 \mathrm{~Hz} /$ pixel, Tempo de Aquisição: 1 minutos e 41 segundos;

A segunda sequência (coronal intermediária ponderada em T2 - TSE com supressão de gordura) foi a escolhida para avaliar a extrusão, no plano incluindo dois marcadores cutâneos posicionados previamente à realização da ultrassonografia (Figura $10)$. 


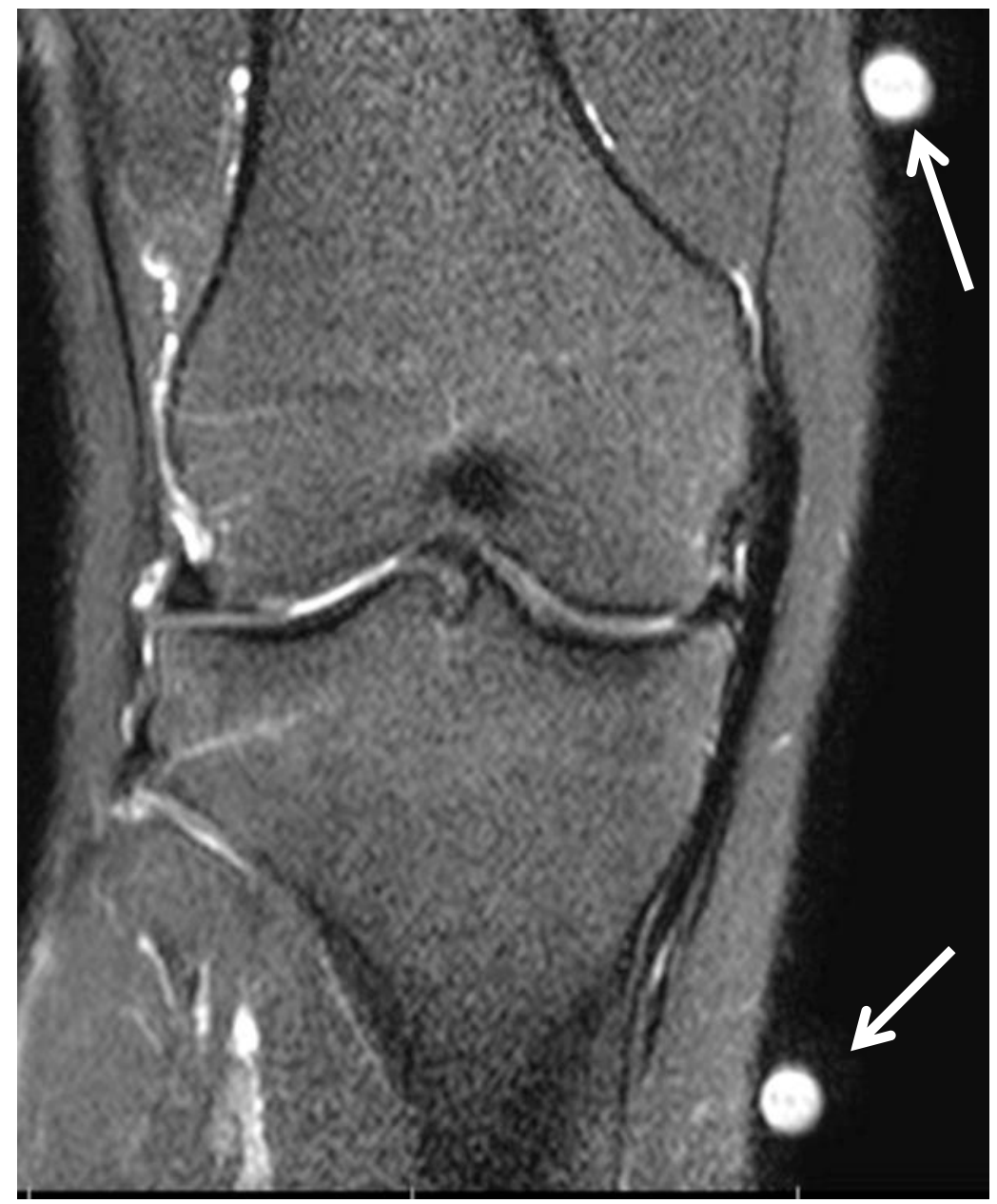

Figura 10. RM do joelho de um participante masculino de 43 anos. A figura ilustra uma sequência coronal intermediária ponderada em T2 - TSE com supressão de gordura, no plano incluindo os dois marcadores cutâneos posicionados previamente à ultrassonografia (setas). Este foi o plano de mensuração da extrusão meniscal, quando presente.

\subsection{Radiografia convencional}

Logo após a realização da ressonância magnética o paciente foi acompanhado pelo medico examinador até a sala de Raio-X, onde foi realizada incidência radiográfica única anteroposterior de ambos os joelhos, em ortostase com carga e apoio bipodal, utilizando chassi do CR de 35 x $43 \mathrm{~cm}$. Mesmo com a dose de radiação sendo mínima 
para exames de extremidades, durante a execução do exame as demais partes do corpo do paciente foram protegidas com coletes de chumbo (Figura 11). Essas radiografias em apoio bipodal foram usadas para avaliar a presença ou ausência de sinais radiográficos de osteoartrite do joelho, através da classificação de Kellgren \& Lawrence, 1957 (Tabela 1) (Kellgren\& Lawrence, 1957; Allen DM et al., 2010).

Tabela 1. Critérios radiográficos para classificação de osteoartrose

\begin{tabular}{|c|c|c|c|c|c|}
\hline $\begin{array}{l}\text { Graduação } \\
\text { radiográfica }\end{array}$ & 0 & I & II & III & IV \\
\hline Classificação & Normal & Duvidoso & Médio & Moderado & Grave \\
\hline Descrição & $\begin{array}{l}\text { Ausência } \\
\text { de } \\
\text { achados } \\
\text { de OA }\end{array}$ & $\begin{array}{l}\text { Mínimo } \\
\text { osteófito, } \\
\text { significância } \\
\text { duvidosa }\end{array}$ & $\begin{array}{l}\text { Osteófito } \\
\text { definitivo, } \\
\text { espaço } \\
\text { articular } \\
\text { normal }\end{array}$ & $\begin{array}{l}\text { Moderada } \\
\text { redução do } \\
\text { espaço } \\
\text { articular }\end{array}$ & $\begin{array}{l}\text { Grande redução } \\
\text { do espaço } \\
\text { articular e } \\
\text { osteoesclerose } \\
\text { subcondral }\end{array}$ \\
\hline
\end{tabular}
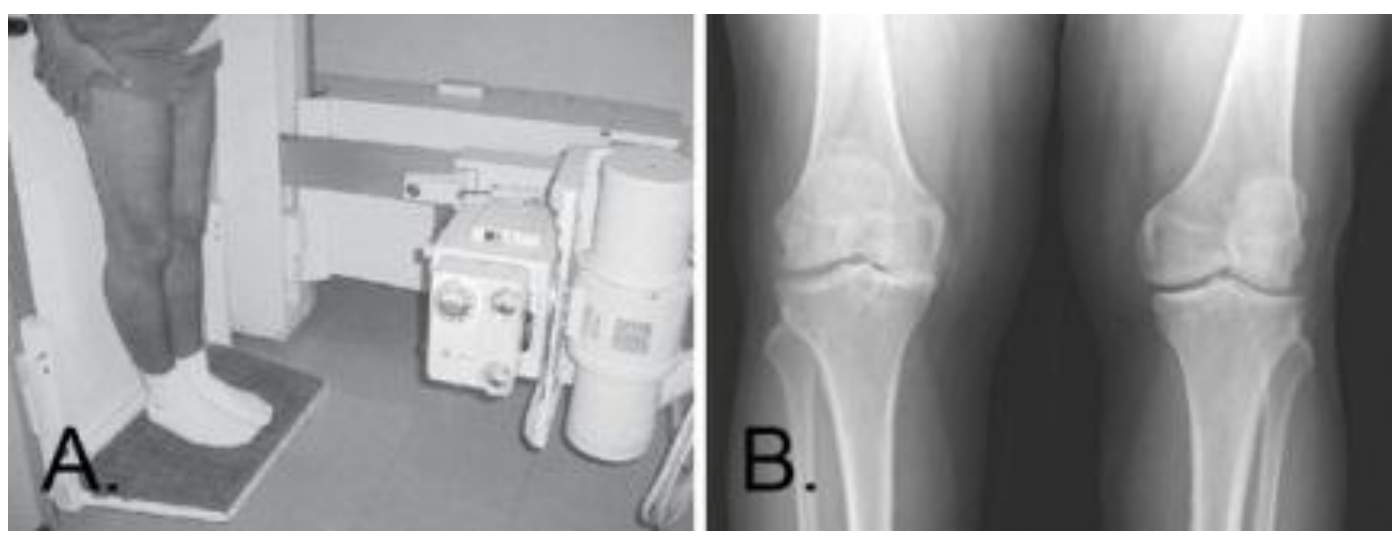

Figura 11. A) Radiografia realizada de ambos os joelhos em incidência anteroposterior em ortostase, com carga e apoio bipodal. B) Imagem radiográfica adquirida. 


\subsection{Ressonância Magnética e Ultrassonografia - Interpretação}

Um mês depois de todos os exames concluídos, dois radiologistas com experiência de 6 e 11 anos (leitor 1 e leitor 2) em diagnóstico por imagem do sistema musculoesquelético mediram de forma independente a extrusão do menisco medial usando as imagens ultrassonográficas adquiridas no eixo longitudinal. Os leitores não tiveram acesso aos dados clínicos dos pacientes, nem às imagens de ressonância magnética.

Uma linha paralela ao eixo longitudinal (craniocaudal), chamada de linha de base, foi traçada na margem óssea do platô tibial medial, servindo como referência para medir a extrusão meniscal.

Um mês após as mensurações ultrassonográficas, os mesmos leitores, novamente cegos aos dados clínicos e às medidas anteriormente adquiridas no US, avaliaram de forma independente a extrusão meniscal medial pela RM usando a sequência coronal intermediária ponderada em T2 - TSE com supressão de gordura, no corte que incluía ambos os marcadores cutâneos. Novamente uma linha paralela ao eixo longitudinal (linha de base) foi traçada nas imagens coronais da RM, na porção mais externa da margem óssea do platô tibial medial, servindo de referência para medir a extrusão meniscal (Crema et al., 2012; Roemer et al., 2009).

Para as avaliações realizadas tanto nas imagens de ultrassom como de RM, a medida da extrusão meniscal foi considerada a distância perpendicular em milímetros entre a linha de referência traçada no eixo longitudinal (coronal) e a margem exterior do corpo do menisco medial (Figura 12). 

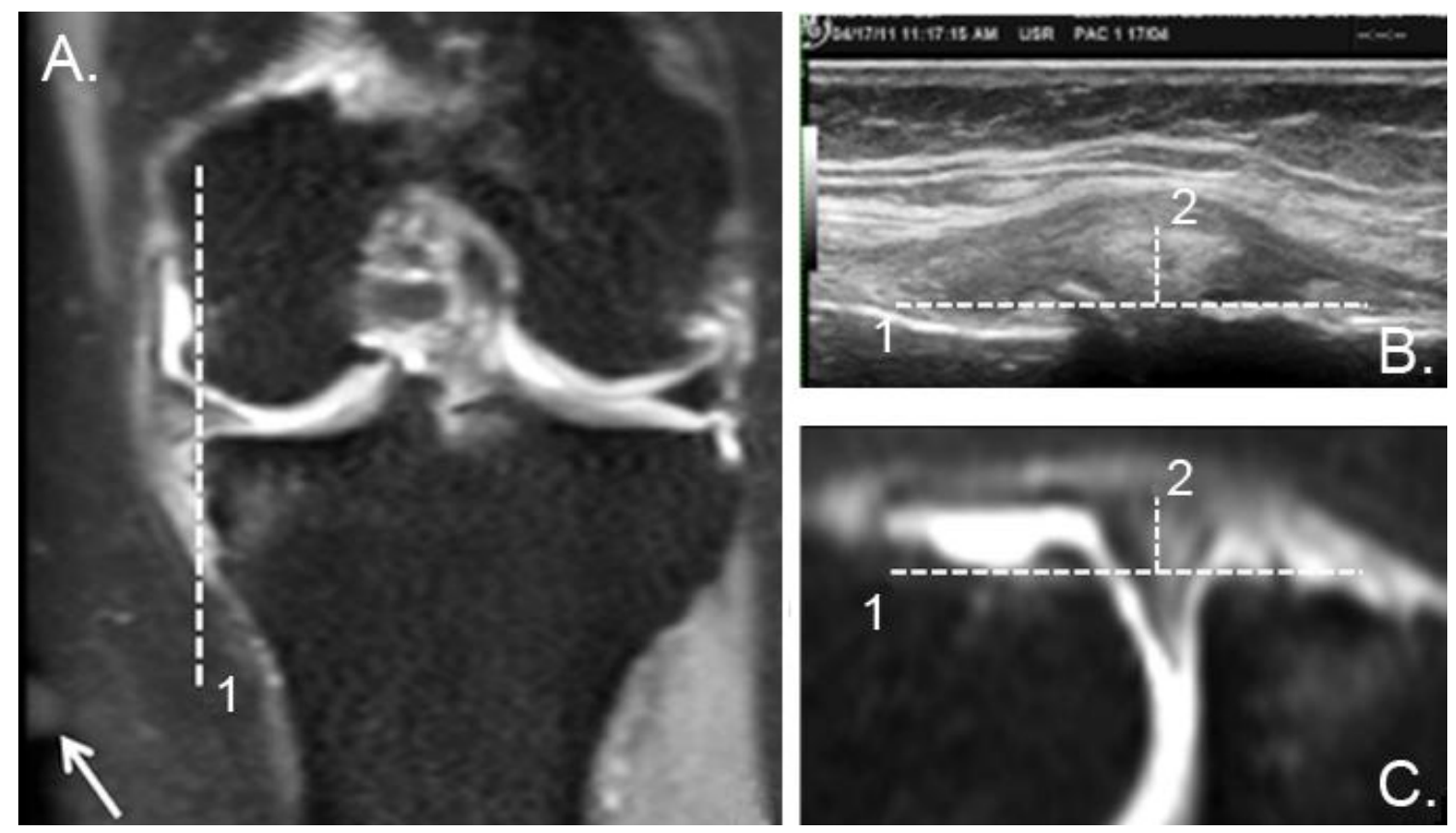

Figura 12. Pontos de referência de medida do joelho. A e C) Na RM, a sequência coronal intermediária ponderada em T2 -TSE com supressão de gordura, mostra o posicionamento da linha de base (linha tracejada 1) e da linha perpendicular (linha tracejada 2) traçada para mensurar a extrusão meniscal. Marcador cutâneo (seta). B) Imagem ultrassonográfica correspondente do mesmo joelho adquirida no eixo longitudinal mostra a linha de base (linha tracejada 1) e a linha perpendicular (linha tracejada 2) onde a mensuração foi realizada.

Para melhorar a reprodutibilidade, as medidas da extrusão meniscal avaliadas nas imagens de RM e US, foram realizadas sistematicamente, incluindo os osteófitos tibiais, quando estes estavam presentes. Todas as medidas foram realizadas em monitores com calipers graduados em milímetros $(\mathrm{mm})$ utilizando uma workstation (eFilmversion 3.4.0, Merge Healthcare).

Devido ambas as abordagens semiquantitativas e quantitativas das mensurações da extrusão meniscal já terem sido previamente aplicadas na literatura para avaliar a 
extrusão meniscal (Crema et al., 2012; Brody et al., 2006; Roemer et al., 2009), decidimos validar as duas abordagens.

A avaliação semiquantitativa refere-se à distribuição das medidas da extrusão meniscal dentro de um sistema de classificação proposto para determinar a sua gravidade ou extensão; assim sendo quatro graus de extrusão meniscal foram propostos: grau $0(<2 \mathrm{~mm})$, grau $1(\geq 2 \mathrm{~mm} \mathrm{e}<4 \mathrm{~mm})$, grau $2(\geq 4 \mathrm{~mm})$.

A avaliação quantitativa realizada neste estudo se refere simplesmente às medidas diretas e absolutas da extrusão meniscal realizadas em milímetros (Figuras 13, 14 e 15).

Após essa primeira etapa de validação o Leitor 1 avaliou as imagens ultrassonográficas adquiridas em ortostase, de forma independente das medidas realizadas anteriormente, utilizando a mesma forma de graduação quantitativa e semiquantitativa. Em seguida o Leitor 1 e o Leitor 2 identificaram e classificaram, em consenso, por meio dos exames de ressonância magnética as lesões meniscais encontradas em cada joelho. 

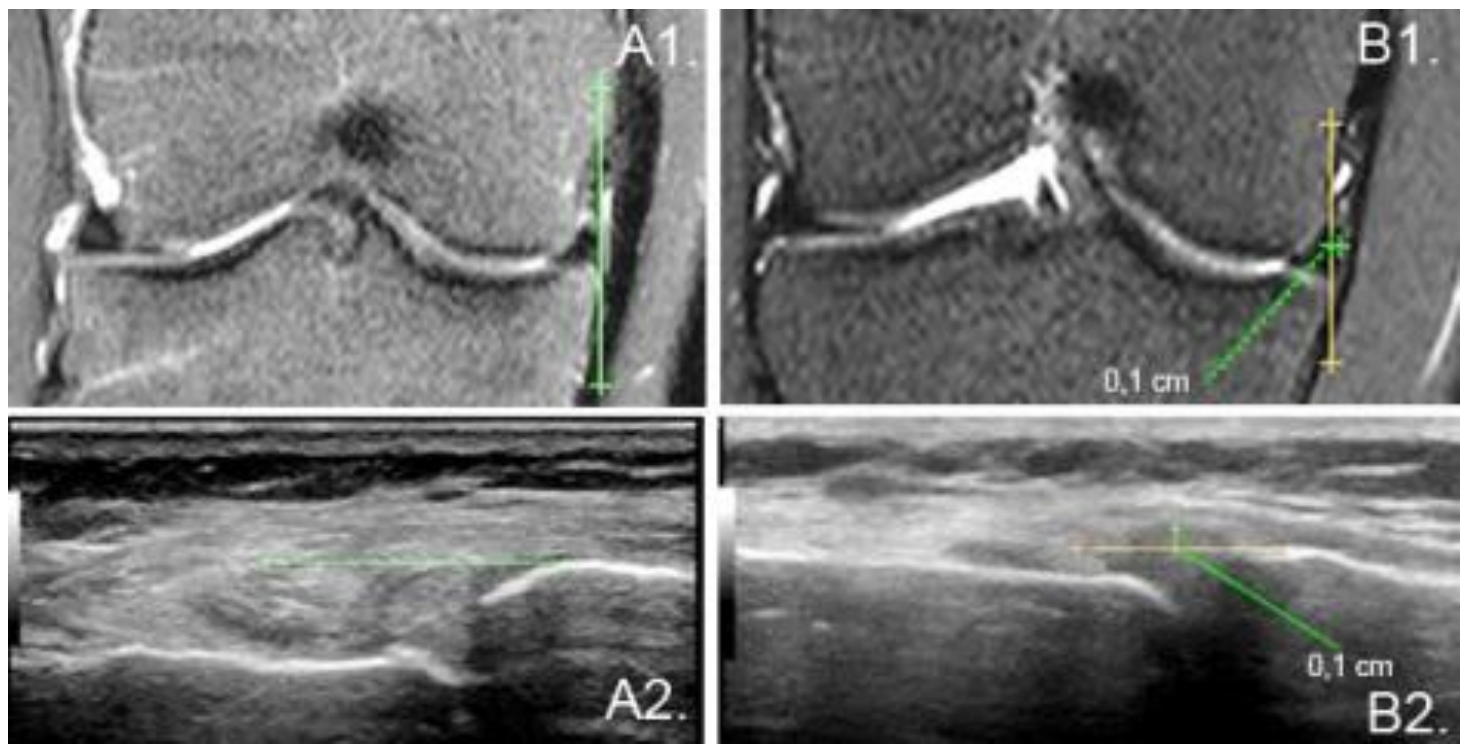

Figura 13. Avaliação da extrusão meniscal. Grau 0 ( $<2$ mm). Participante A com dor crônica no joelho, mostra linha de base traçada na RM (A1) e na ultrassonografia (A2), e à ausência de extrusão meniscal. Participante B com dor crônica no joelho, também com linha de base traçada na RM (B1) e na ultrassonografia (B2), mostra linha perpendicular medindo o valor da extrusão meniscal, que em ambos US e RM foi menor do 2 mm. 

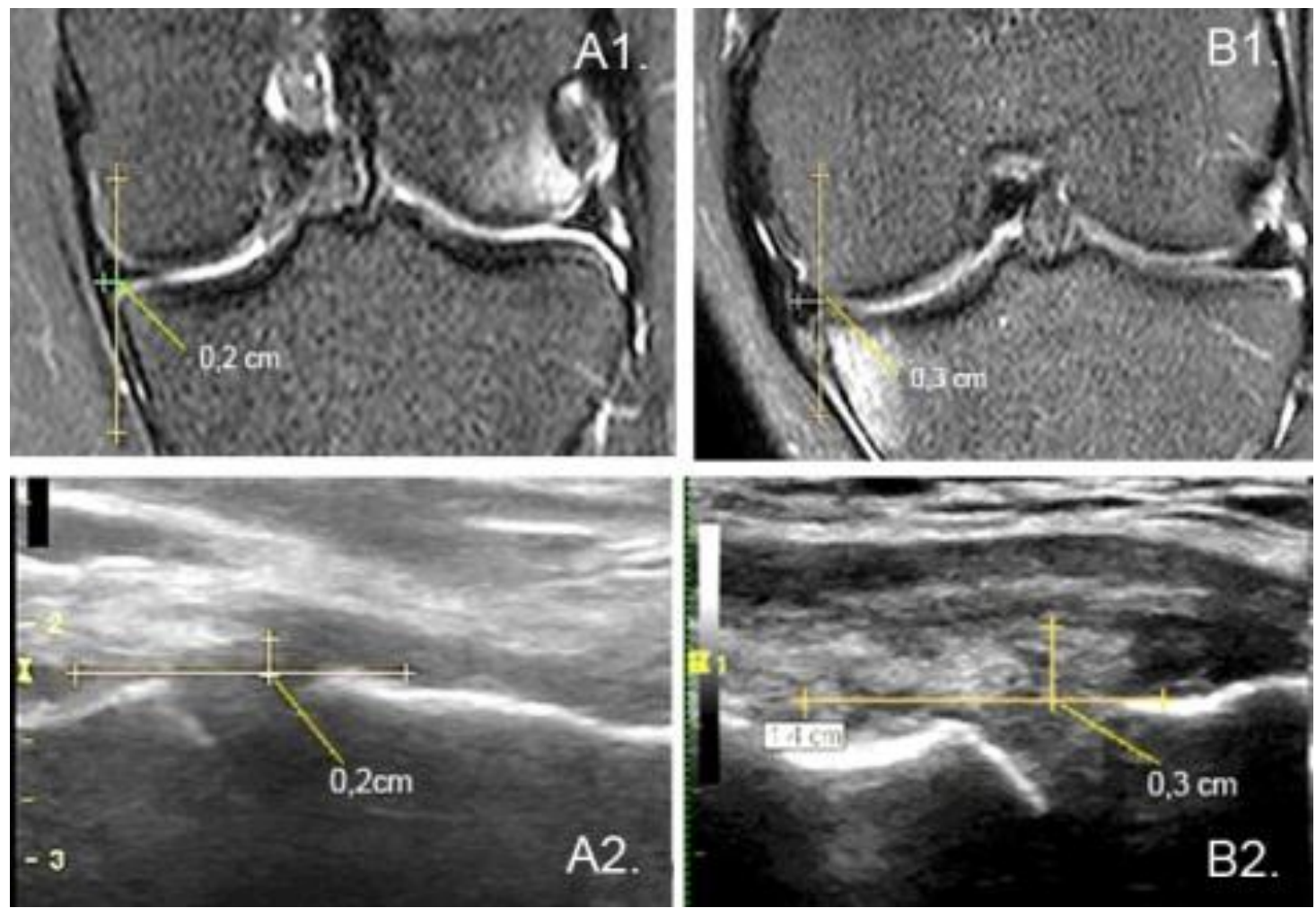

Figura 14. Avaliação da extrusão meniscal. Grau 1 ( $\geq 2 \mathrm{~mm}$ e $<4 \mathrm{~mm})$. Participante A com dor crônica no joelho, linha de base traçada na RM (A1) e na ultrassonografia (A2), linha perpendicular medindo o valor da extrusão meniscal, que na US e na RM foi de 2 mm. Participante B com dor crônica no joelho, linha de base traçada na RM (B1) e na ultrassonografia (B2), mostra linha perpendicular medindo o valor da extrusão meniscal, que em ambos US e RM foi de $3 \mathrm{~mm}$. 

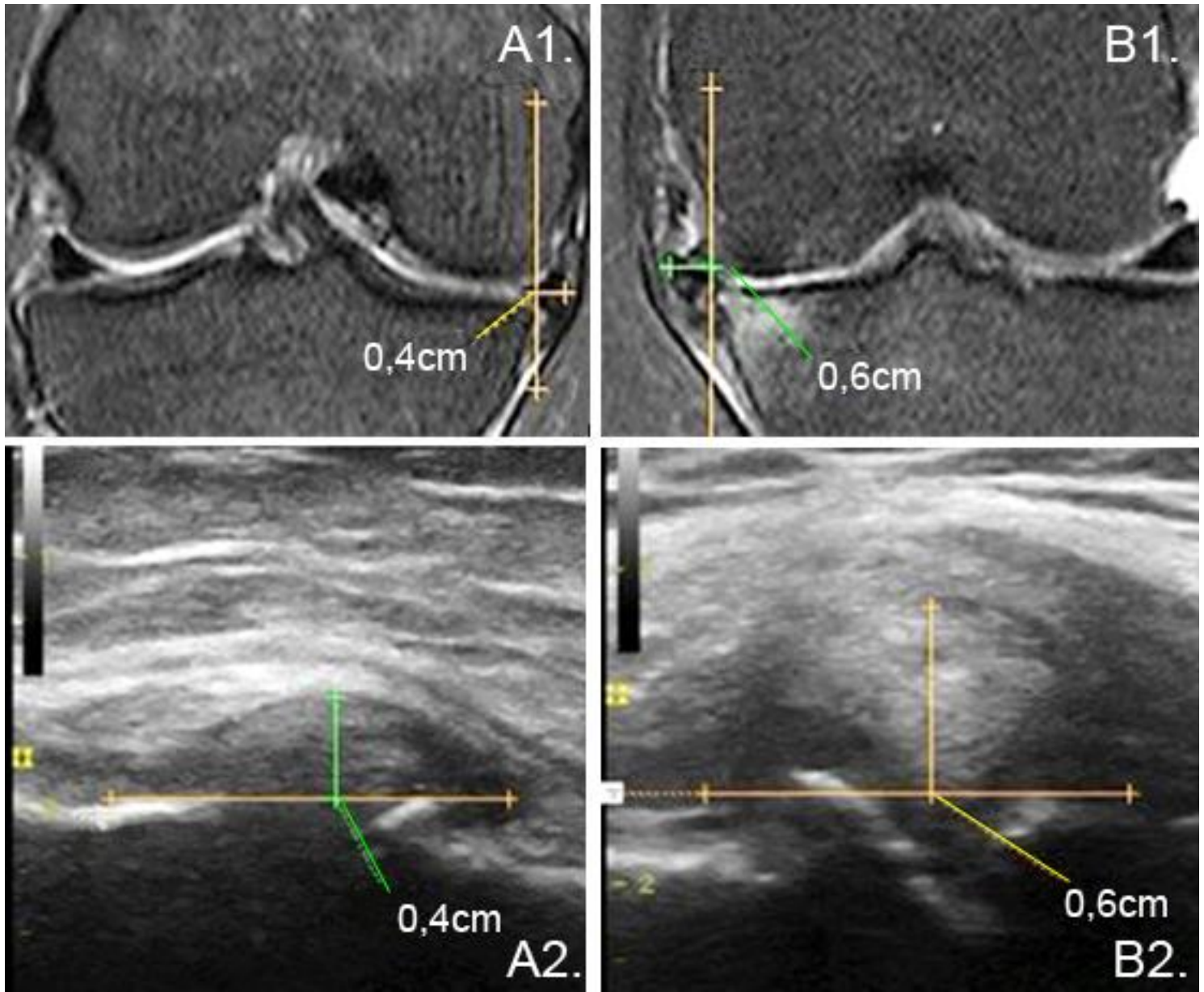

Figura 15. Avaliação da extrusão meniscal. Grau 2 ( $\geq 4 \mathrm{~mm})$. Participante A com dor crônica no joelho, linha de base traçada na RM (A1) e na ultrassonografia (A2), linha perpendicular medindo o valor da extrusão meniscal, que em ambos US e RM foi de 4 mm. Participante B com dor crônica no joelho, linha de base traçada na RM (B1) e na ultrassonografia (B2), mostra linha perpendicular medindo o valor da extrusão meniscal, que em ambos US e RM foi maior que 4mm (6 mm). 


\subsection{Análise Estatística}

A correlação intra e interobservador para a avaliação dos graus de extrusão meniscal em cada método de imagem (US e RM), e a concordância entre os dois

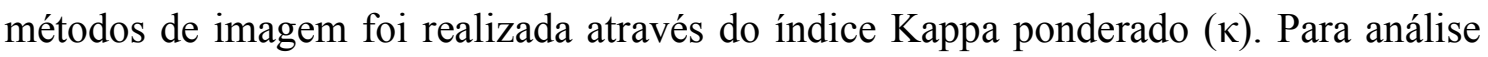
do desempenho diagnóstico do US na avaliação da extrusão meniscal, quando comparado com a RM o padrão de referência, foram calculadas: sensibilidade, especificidade e acurácia.

Para avaliar os efeitos da mensuração realizada em decúbito e em ortostase foi utilizada a correlação intraclasse (CIC) com intervalos de confiança (IC) de 95\%. Foi utilizado o teste pareado de Wilcoxon para avaliar a diferença nos valores de extrusão dos indivíduos nas posições decúbito e ortostase. Para analisar os resultados obtidos pela mensuração entre grupos com presença e ausência de lesão foi utilizado o teste $\mathrm{T}$ não pareado (considerando resultados estatisticamente significantes quando $\mathrm{p}<0,05$ ); e para avaliar a frequência dos graus mensurados em cada grupo (presença e ausência de lesão) foi utilizado o teste qui-quadrado $\left(\mathrm{x}^{2}\right)$, também sendo considerado resultado significante quando $\mathrm{p}<0,05$. $\mathrm{O}$ teste $\mathrm{T}$ também foi utilizado para comparar os valores de extrusão meniscal entre os grupos com presença e ausência de dor, e os grupos com presença e ausência de osteoartrite radiográfica. Para correlação dos graus de extrusão entre os diferentes tipos de lesão foi utilizado o teste de Kruskal-Wallis com pós teste de Dunns (considerado resultado significante quando $\mathrm{p}<0,05$ ). Foi utilizada a correlação de Spearman para verificar a relação entre as medidas de extrusão meniscal com a idade e IMC dos voluntários.

A análise estatística foi realizada utilizando os softwares SPSS versão 20 (IBM, Armonk, New York) e GraphPadPrism versão 5 (GraphPad Software, San Diego, California). 


\section{RESULTADOS}

\subsection{Validação da avaliação de extrusão meniscal medial por US e RM}

A análise para o mesmo examinador da avaliação semiquantitativa de extrusão meniscal medial entre US e a RM apresentou o índice Kappa ponderado ( $\kappa)$ moderado conforme apresentado na Tabela 2.

Tabela 2. Comparação das graduações de extrusão meniscal medial entre ultrassonografia e ressonância magnética (RM) para o mesmo examinador.

\begin{tabular}{|c|c|c|c|c|}
\hline \multirow[b]{2}{*}{$\begin{array}{c}\text { Observador/Graduação } \\
\text { RM }\end{array}$} & \multicolumn{3}{|c|}{ Ultrassonografia } & \multirow[b]{2}{*}{ Total } \\
\hline & Grau 0 & Grau 1 & Grau 2 & \\
\hline \multicolumn{5}{|l|}{ Leitor $\mathbf{1}^{\mathrm{a}}$} \\
\hline Grau 0 & 46 & 8 & 1 & 55 \\
\hline Grau 1 & 11 & 14 & 3 & 28 \\
\hline Grau 2 & 0 & 3 & 7 & 10 \\
\hline Total & 57 & 25 & 11 & 93 \\
\hline \multicolumn{5}{|l|}{ Leitor $2^{b}$} \\
\hline Grau 0 & 47 & 7 & 1 & 55 \\
\hline Grau 1 & 10 & 15 & 2 & 27 \\
\hline Grau 2 & 0 & 2 & 9 & 11 \\
\hline Total & 57 & 24 & 12 & 93 \\
\hline
\end{tabular}

a: $\kappa=0,57$; IC 95\%: 0,41-0,73

b: $\kappa=0,64$; IC 95\%: 0,48-0,80

A Tabela 3 apresenta a análise interobservador para cada método de imagem (US e RM), com índice Kappa substancial para a RM ( $\kappa=0,70 ; \mathrm{IC} 95 \%$ : 0,55-0,85) e excelente para o US $(\kappa=0,98$; IC 95\%: 0,94-1,00). Para a análise intraobservador, o Leitor 1 apresentou concordância substancial para RM $(\kappa=0,70$; IC 95\%=0,45-0,94) e quase perfeita para US $(\kappa=0,99 ; \mathrm{IC}=95 \%, 0,76-1,0)$ (Tabela 4). Os achados foram semelhantes para a concordância intraobservador do Leitor 2. Para Leitor 2, tanto para a $\mathrm{RM}(\kappa=0,68$; IC $95 \%=0,42-0,93)$ quanto para o US $(\kappa=0,91$; IC $95 \%=0,66-1,0)$ a concordância foi expressiva. 
Tabela 3. Análise interobservador para avaliação de extrusão meniscal medial em cada método de imagem. $(\mathrm{RM}=$ ressonância magnética $)$

\begin{tabular}{c|c|c|c|c}
\hline \multirow{2}{*}{ Leitor 1 } & \multicolumn{3}{|c|}{ Leitor 2 } & Total \\
\cline { 2 - 5 } RM $^{\mathbf{a}}$ & Grau 0 & Grau 1 & Grau 2 & 55 \\
Grau 0 & & & 0 & 27 \\
Grau 1 & 47 & 8 & 1 & 11 \\
Grau 2 & 0 & 18 & 9 & 57 \\
\hline Ultrassonografia $^{\mathbf{b}}$ & 57 & 0 & 0 & 24 \\
Grau 0 & 0 & 24 & 0 & 12 \\
Grau 1 & 0 & 1 & 11 & \\
Grau 2 & & & & \\
\hline
\end{tabular}

a: Kappa= 0,70; IC: 0,55-0,85

b: Kappa= 0,98; IC: 0,94-1,00

Tabela 4. Análise intraobservador para avaliação de extrusão meniscal medial em cada método de imagem. ( $R M=$ ressonância magnética, $U S=$ ultrassonografia $)$

Leitor $1 \quad$ Segunda Leitura

\begin{tabular}{|c|c|c|c|c|}
\hline RM/ Primeira Leitura ${ }^{a}$ & Grau 0 & Grau 1 & Grau 2 & Total \\
\hline Grau 0 & 8 & 4 & 2 & 14 \\
\hline Grau 1 & 8 & 6 & 2 & 16 \\
\hline Grau 2 & 3 & 1 & 0 & 34 \\
\hline Leitor 1 & \multicolumn{3}{|c|}{ Segunda Leitura } & \\
\hline US/ Primeira Leitura ${ }^{b}$ & Grau 0 & Grau 1 & Grau 2 & Total \\
\hline Grau 0 & 24 & 0 & 0 & 24 \\
\hline Grau 1 & 0 & 10 & 0 & 10 \\
\hline Grau 2 & 0 & 0 & 5 & 5 \\
\hline
\end{tabular}

\begin{tabular}{|c|c|c|c|c|}
\hline Leitor 2 & & unda Lei & & \\
\hline $\mathbf{R M} /$ Primeira Leitura ${ }^{\mathrm{C}}$ & Grau 0 & Grau 1 & Grau 2 & Total \\
\hline Grau 0 & 19 & 2 & 0 & 21 \\
\hline Grau 1 & 4 & 10 & 0 & 14 \\
\hline Grau 2 & 0 & 1 & 3 & 4 \\
\hline
\end{tabular}




\begin{tabular}{|c|c|c|c|c|}
\hline Leitor 1 & & unda Lei & & \\
\hline US/ Primeira Leitura ${ }^{d}$ & Grau 0 & Grau 1 & Grau 2 & Total \\
\hline Grau 0 & 24 & 0 & 0 & 24 \\
\hline Grau 1 & 0 & 10 & 0 & 10 \\
\hline Grau 2 & 0 & 2 & 3 & 5 \\
\hline
\end{tabular}

a: $\kappa=0,70 ;$ IC $95 \%=0,45-0,94 ; b: \kappa=0,99 ;$ IC $=95 \%, 0,76-1,0$

c: $\kappa=0,68 ;$ IC $95 \%=0,42-0,93 ; \mathrm{d}: \kappa=0,91 ;$ IC $95 \%=0,66-1,0$

Para mensurações quantitativas, a média $( \pm \mathrm{DP})$ de extrusão meniscal para o Leitor 1 foi de $1,4 \pm 1,5 \mathrm{~mm}$ no US e de 1,4 $\pm 1,4 \mathrm{~mm}$ na RM. A extrusão meniscal média para Leitor 2 foi de $1,4 \pm 1,5 \mathrm{~mm}$ no US e $1,3 \pm 1,4 \mathrm{~mm}$ na RM. Quando utilizados os valores absolutos de extrusão meniscal medial (avaliação quantitativa) mensurados pelo mesmo leitor, a comparação entre a avaliação por US e por RM apresentou resultados substanciais para ambos examinadores (Leitor 1 e 2), com coeficiente de correlação intraclasse de 0,70 (IC 95\%=0,58-0,79) e 0,73 (IC 95\%= 0,58-0,79) respectivamente.

A avaliação por US apresentou excelente desempenho diagnóstico na detecção da extrusão meniscal medial ao se comparar com a RM como padrão de referência, com acurácia de $92 \%$ e $95 \%$ para cada examinador (Tabela 5). 
Tabela 5. Desempenho diagnóstico da ultrassonografia na detecção da extrusão meniscal medial comparada com a ressonância magnética

\begin{tabular}{c|c|c|c|c|c|c}
\hline & \multicolumn{2}{|c|}{ Ressonância Magnética } & \multicolumn{3}{|c}{} \\
\hline Ultrassom & Normal & Extruso & Total & $\begin{array}{c}\text { Sensibilidade } \\
(\%)\end{array}$ & $\begin{array}{c}\text { Especificidade } \\
(\%)\end{array}$ & $\begin{array}{c}\text { Acurácia } \\
(\%)\end{array}$ \\
\hline Leitor 1 & 79 & 3 & 82 & 95 & 70 & 92 \\
Normal & 4 & 7 & 11 & & & \\
Extruso & 83 & 10 & 93 & & & \\
\hline Total & & & & 96 & 82 & \\
\hline Leitor 2 & 79 & 2 & 81 & & & \\
Normal & 3 & 9 & 12 & & & \\
Extruso & 82 & 11 & 93 & & & \\
\hline Total & 82 & & & & &
\end{tabular}

Normal= Grau 0; Extruso $=$ Grau 1 e 2

\subsection{Mensuração de extrusão meniscal medial com e sem carga por meio do US}

Na avaliação da extrusão meniscal medial por US buscamos avaliar se a avaliação feita em decúbito dorsal (sem carga) e na em ortostase (com aplicação de carga do peso corporal) apresentavam diferenças entre si. Os valores médios de extrusão meniscal foram de $1,5 \pm 1,8 \mathrm{~mm}$ para posição em decúbito e 1,65 \pm 1,8 mm para ortostase. Houve diferença significativa destes valores médios de extrusão meniscal entre as duas posições $(\mathrm{p}=0,0002)$.

Na posição em decúbito, quando comparados os valores médios das medidas de extrusão meniscal medial da ultrassonografia foi possível observar diferença estatisticamente significativa $(\mathrm{p}<0,0001)$ entre o grupo com presença de lesão do menisco detectada na ressonância magnética $(2,281 \pm 2,03 \mathrm{~mm})$ e o grupo com ausência de lesão $(0.55 \pm 0.68 \mathrm{~mm})$ (Figura 16). 


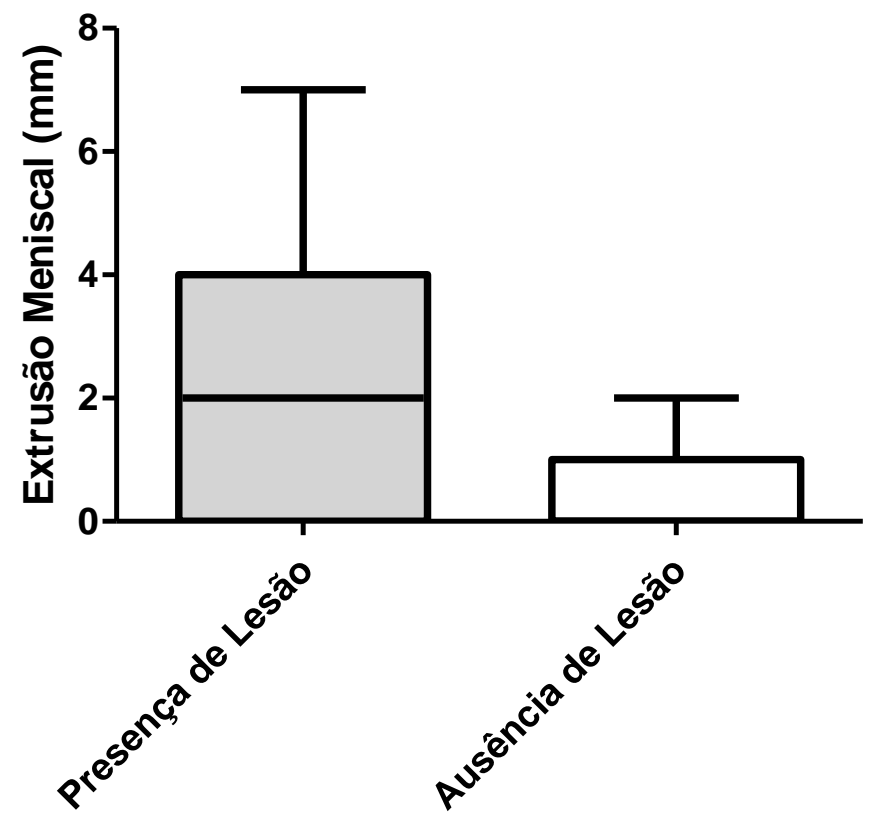

Figura 16. Mensuração da Extrusão Meniscal Medial (em mm) por meio da ultrassonografia de acordo com a presença ou ausência de lesão do menisco detectada na ressonância magnética.

A Tabela 6 e a Figura 17 apresentam as frequências da mensuração da extrusão meniscal (em milímetros) em cada joelho de acordo com a presença ou ausência de lesão (confirmadas via RM). Foi encontrada diferença estatisticamente significativa entre os grupos $\left(x^{2}: 33,662, p<0,0001\right)$. Além disso, todos os meniscos com extrusões $\geq$ $3 \mathrm{~mm}$ apresentavam lesão do menisco na RM. 
Tabela 6. Frequência da distribuição dos graus de extrusão meniscal na ultrassonografia de acordo com a presença ou ausência de lesão do menisco na ressonância magnética.

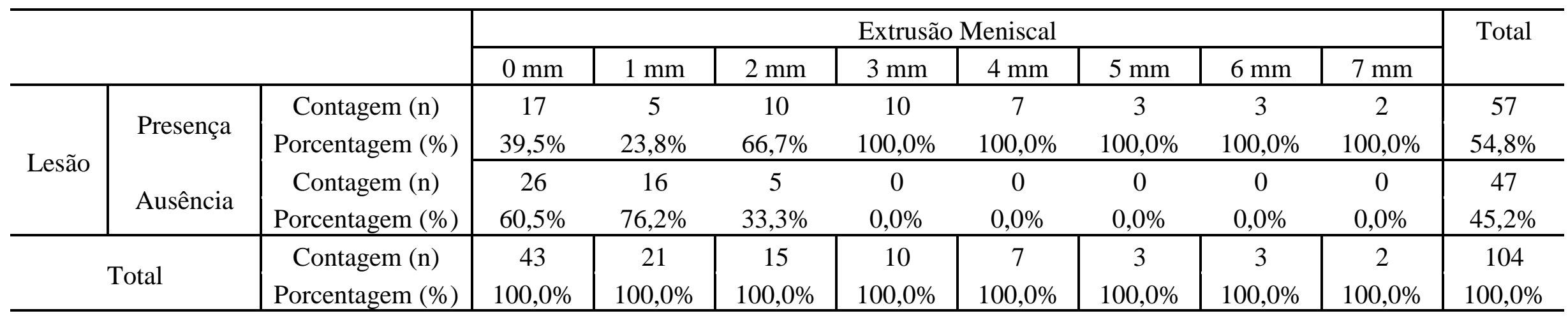




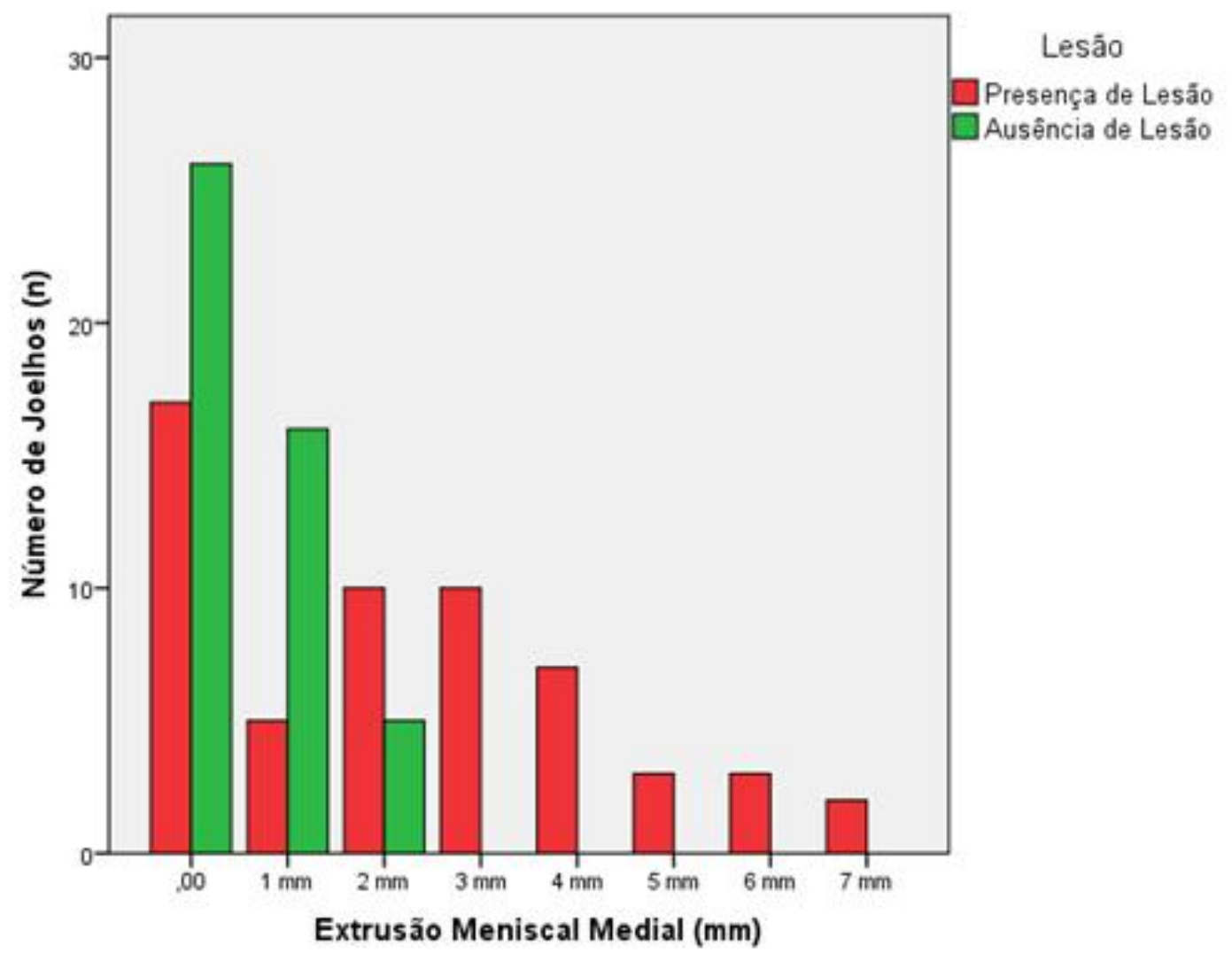

Figura 17. Distribuição das medidas de extrusão meniscal medial mensurada pela ultrassonografia de acordo com a presença ou ausência de lesão do menisco na ressonância magnética.

\subsection{Avaliação dos fatores associados à extrusão meniscal medial}

Foram comparados os valores médios de extrusão de acordo com o tipo de lesão encontrada: Íntrego (sem lesão), Flap, Radial de Raíz Posterior (Radial RP), Horizontal, Vertical, Maceração, Complexa e Alça de Balde. A Tabela 7 apresenta os valores de acordo com cada um destes tipos. Houve diferença estatisticamente significativa entre os seguintes grupos (Kruskal-Wallis $=40,85 ; \mathrm{p}<0,0001$ ): os valores de extrusão meniscal do grupo Íntegro foi significativamente menor em relação aos grupos Flap, Radial RP e Maceração ( $\mathrm{p}<0,0001$ ). Quando comparados entre si, os valores de extrusão dos diferentes tipos de lesão não diferiram entre si. A única exceção ocorreu 
para a lesão Alça Balde que foi estatisticamente menor que as lesões Radial RP e Maceração (p < 0,0001).

Tabela 7. Extrusão meniscal medial (em $\mathrm{mm}$ ) mensurada por meio da ultrassonografia de acordo com o tipo de lesão encontrada nas imagens de ressonância magnética.

\begin{tabular}{ccc}
\hline Tipo de Lesão & Média & Desvio Padrão \\
\hline Íntrego & 0,55 & 0,68 \\
Flap & 2,33 & 1,83 \\
Radial Raiz Posterior & 3,38 & 2,02 \\
Horizontal & 1,43 & 1,27 \\
Vertical & 2,20 & 2,68 \\
Maceração & 3,66 & 2,65 \\
Complexa & 2,66 & 2,94 \\
Alça de Balde & 0,20 & 0,44 \\
\hline
\end{tabular}

Dos 104 indivíduos, 42 não apresentavam dor e 62 tinham sintomas dolorosos. Quando comparados os graus de extrusão meniscal de acordo com a presença ou ausência de dor, o grupo com dor apresentou valores médios de extrusão meniscal medial significativamente maiores que o grupo sem dor (Com dor: 2,60 $\pm 1,95 \mathrm{~mm}$; Sem dor: $0,63 \pm 1,02 \mathrm{~mm} ; \mathrm{p}<0,0001)$. 


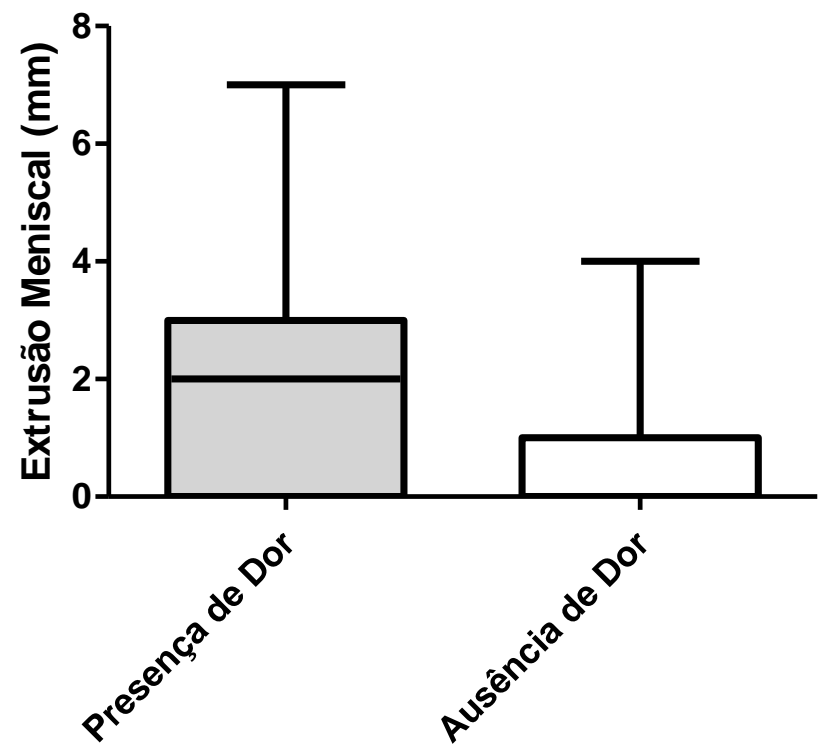

Figura 18. Graduação da Extrusão Meniscal Medial (em mm) de acordo com a presença ou ausência de dor.

Dos 104 pacientes do estudo, 33 (23,6\%) apresentavam osteoartrite radiográfica (KL grau $\geq 2$ ). Ao classificar os grupos de acordo com a presença ou ausência de osteoartrite radiográfica, foi observado que o grupo com OA apresentou valores médios de extrusão meniscal medial significativamente maiores que o grupo sem OA (osteoartrite: 2,36 $\pm 2,04 \mathrm{~mm}$; Ausência de osteoartrite: $1,10 \pm 1,51 \mathrm{~mm}$; $\mathrm{p}=0,0011$ ). 


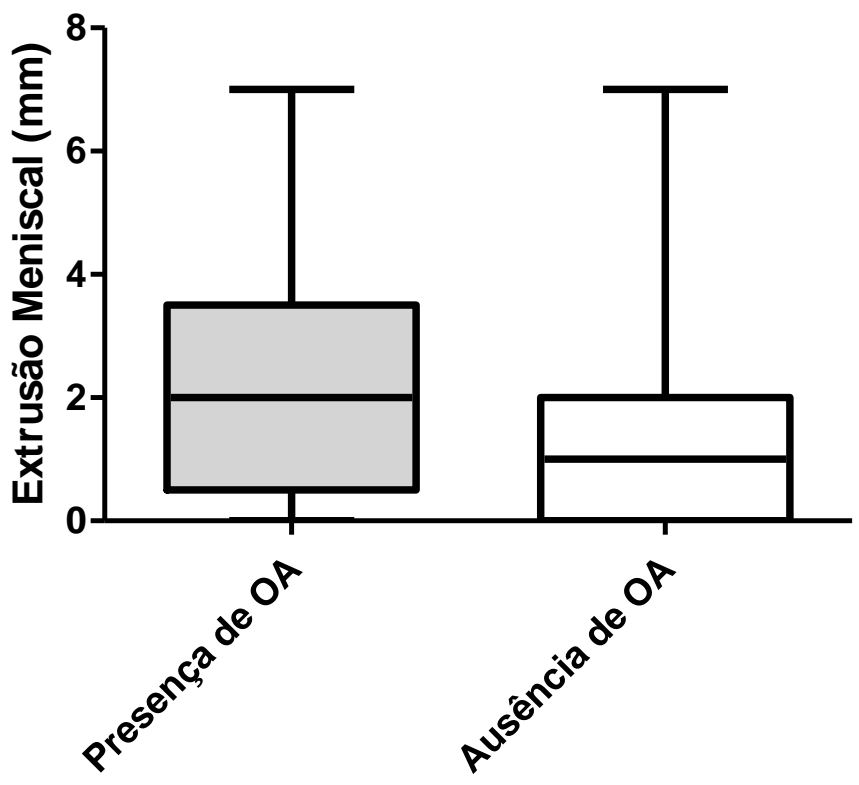

\section{Figura 19. Mensuração da Extrusão Meniscal Medial (em mm) pela ultrassonografia de acordo com a presença ou ausência de osteoartrite (OA)radiográfica.}

A idade dos indivíduos com ausência de sinais de OA foi de 37,33 $\pm 12,25$ anos, enquanto que nos indivíduos com sinais de OA foi de 47,04 $\pm 13,95$. Houve diferença estatisticamente significativa entre estes dois grupos $(p=0,0005)$. Foi observada uma correlação moderada $(\mathrm{R}=0,40 ; \mathrm{p}=0,0007)$ entre a idade e a medida de extrusão meniscal no grupo com ausência de OA. Já no grupo com presença de OA houve uma correlação fraca para as mesmas variáveis $(\mathrm{R}=0,34 ; \mathrm{p}=0,04)$. Ao correlacionar as medidas de extrusão meniscal e o IMC dos voluntários, foi encontrada uma fraca correlação, porém significativa $(\mathrm{R}=0,31 ; \mathrm{p}=0,0014)$. 


\section{DISCUSSÃO}

Neste trabalho buscamos avaliar a extrusão meniscal no compartimento femorotibial medial em indivíduos com dor crônica no joelho sob a presença e ausência de carga corpórea. Além disso, objetivou-se a validação deste método de avaliação através da ultrassonografia, utilizando o padrão de referência a ressonância magnética. Desta forma, foi possível avaliar a inter-relação entre as variações da extrusão meniscal com os vários fatores mecânicos e morfológicos que possam estar implicados. Em nossos resultados, foi visto que a US apresentou excelente desempenho diagnóstico para extrusão meniscal, tanto quantitativo quanto semiquantitativo, quando comparada à RM. Foi encontrada diferença entre a avaliação na presença de carga corpórea (ortostase) e na sua ausência (decúbito dorsal). Também foi observado que todos os joelhos com extrusão meniscal de $3 \mathrm{~mm}$ na ultrassonográfica apresentavam lesão meniscal identificável nas imagens de RM.

Em relação à validação do método de US para avaliação da extrusão meniscal, este foi o primeiro estudo que comparou diretamente a avaliação por ultrassonografia com a RM. Embora estudos anteriores tenham utilizadoa ultrassonografia para a avaliação de extrusão meniscal (Verdonket al., 2004; Kawagushi et al., 2012; Acebes et al., 2013; Ko et al., 2007), o uso da US ainda não foi validado em confrontação direta com RM, para que assim possa ser aplicado na prática clínica e na pesquisa.

$\mathrm{Na}$ avaliação semiquantitativa de extrusão (Graus 0,1 e 2 ) houve uma concordância moderada a substancial entre US e RM. Para esta mesma avaliação, foi visto que a US apresentou melhor concordância entre observadores, em relação à RM, tanto na análise intraobservador quanto na interobservador. Tal achado pode estar relacionado ao fato de que apenas uma imagem ultrassonográfica de cada compartimento medial foi armazenada para a mensuração e análise. Além disso, em 
alguns casos de RM, mais de um corte coronal apresentavam ambos os marcadores, possibilitando que as medidas fossem feitas em cortes diferentes.

Na avaliação quantitativa, a média e desvio padrão dos valores de extrusão do US e RM foram semelhantes; e o coeficiente de correlação intraclasse entre as técnicas foi substancial para os dois leitores (resultado semelhante ao da avaliação semiquantitativa).

Tais resultados demonstraram que a US é uma ferramenta confiável. Isto é verdade especialmente em casos de alterações degenerativas moderadas a avançadas no compartimento femorotibial medial, onde as alterações afetam não somente o menisco, mas também tecidos moles adjacentes como o ligamento colateral medial e sinóvia (Kawagushi et al., 2012; Wareluk\&Szopinski, 2012). Além disso, embora exista alguma evidência de que a US pode diagnosticar com precisão rupturas meniscais (Wareluk\&Szopinski, 2012), a possível utilização da ultrassonografia para este fim requer validação e cautela. A RM foi utilizada para a comparação com a US, pois uma de suas principais vantagens é que o contraste e o sinal podem ser manipulados para realçar diferentes tecidos ao redor da articulação, tornando-a o método de imagem de referência amplamente aceito na avaliação de anormalidades das estruturas internas do joelho, incluindo as lesões associadas a extrusão meniscal (Crema et al., 2012; Roemer et al., 2009; Ding et al., 2007; Brody et al., 2006).

O fato da US ser um instrumento de fácil acesso, confiável e ter excelente desempenho diagnóstico na avaliação de extrusão meniscal, sugere que possa ser aplicado não só para a avaliação de deslocamento estático de menisco, como neste estudo, mas também para a avaliação do deslocamento dinâmico de menisco e assim maior correlação com a sintomatologia. 
Na avaliação da extrusão meniscal medial por US feita em decúbito dorsal (sem carga) e em ortostase (com aplicação de carga do peso corporal) os valores de extrusão meniscal da posição ortostática foram significativamente maiores que em decúbito. Em uma avaliação dinâmica utilizando as duas posições, o deslocamento que ocorre na mudança da posição supina para posições de suporte de peso pode ser quantificado. Esta prática é mais difícil de realizar com a RM pela necessidade de um aparelho de campo aberto para adquirir as imagens em ortostase. Como nossos resultados mostraram uma boa confiabilidade do US, assumimos que a avaliação com descarga de peso pode ser viável utilizando este método.

Dois estudos prévios avaliaram extrusão meniscal dinamicamente com US, comparando indivíduos com e sem osteoartrite do joelho (Kawagushiet al., 2012; Acebes et al., 2013), e mostrarama extrusão meniscal é mais frequente nos compartimentos com osteoartrite, agravando-se com a mudança da posição supina para ortostase (deslocamento dinâmico). Nosso estudo apresentou resultados semelhantes. Apesar de que em alguns dos trabalhos citados anteriormente os indivíduos permaneceram na posição ereta por mais tempo e foram submetidos a estresse dinâmico sobre a articulação, o tempo de um minuto utilizado no nosso projeto demonstrou ser suficiente para exibir as alterações nos graus de extrusão meniscal.

Embora esta descoberta sugira algumas possibilidades, o significado real do deslocamento dinâmico do menisco ainda carece de maiores estudos, com por exemplo, se o deslocamento dinâmico dos meniscos afetam o curso da osteoartrite de forma diferente daqueles em que o deslocamento não ocorre.

Na avaliação por US na posição em decúbito, a extrusão meniscal dos indivíduos com presença de lesão confirmada pela RM foi significativamente maior do que de indivíduos sem lesão detectada. Também foi observado que a partir de $3 \mathrm{~mm}$ de 
extrusão do menisco medial, 100\% dos casos apresentam lesão meniscal na RM (Tabela 6), enquanto a partir de $2 \mathrm{~mm}$ de extrusão, um terço dos pacientes apresentavam lesão meniscal (66,7\%). Tal observação nos permite concluir que extrusões $\geq 3 \mathrm{~mm}$ pela US permitem detectar a presença de lesões meniscais com segurança. Stehlinget al. (2012) definiram em sua metodologia que um menisco normal apresenta uma extrusão menor que $3 \mathrm{~mm}$, e uma extrusão maior que $3 \mathrm{~mm}$ foi estabelecida como anormal. Não foi encontrada na literatura nenhum estudo validando esta medida, sendo até então uma classificação empírica. Nossos achados sugerem que a extrusão meniscal acima de 3 mm confirma a existência de lesão meniscal.

A carga entre o fêmur e a tíbia gera uma compressão sobre o menisco que, devido à seu aspecto transversal em cunha, resulta num vetor radial dirigido para o exterior (Vedi et al., 1999). Sob condições compressivas, a distância entre os cornos do menisco aumenta, resultando na compressão das regiões periféricas e, consequentemente seu deslocamento (Tibeskuet al., 2004). Boxheimeret al. (2004) examinaram indivíduos assintomáticos por RM de $1.5 \mathrm{~T}$ e encontraram que o movimento meniscal foi mais proeminente no corno anterior do menisco medial com o joelho flexionado a $90^{\circ}$, em rotação interna ou externa, na posição de decúbito.

Por outro lado, em estudo por RM realizado por Boxheimeret al. (2006), pacientes com rupturas meniscais apresentavam deslocamentos de $3 \mathrm{~mm}$ em $42 \%$ dos meniscos com rupturas. Meniscos extrusos comumente apresentam lesões complexas, radiais ou longitudinais (Stehlinget al., 2012).

Do total de 104 meniscos estudados, 15 aumentaram o grau da extrusão durante a mudança de posição de decúbito para ortostase, desses 15 meniscos que sofreram alteração da posição: 7 eram meniscos normais, e 8 eram meniscos lesados (aproximadamente 50/50). A lesões meniscais estavam assim distribuídas: 5 rupturas / 
flap (62.5\%); 1 ruptura complexa (flap + ruptura radial na raiz posterior $-12.5 \%) ; 1$ ruptura vertical (12.5\%); e 1 menisco macerado (12.5\%). Foi encontrado apenas 1 menisco com lesão na raiz posterior, dentre os que apresentaram alteração da extrusão entre o decúbito e a ortotase.

Dos 89 meniscos que não apresentaram mudanças na sua posição em relação à avaliação em decúbito e ortostase, 47 (52.8\%) tinham lesão, e desses 47 meniscos lesados que não alteraram sua posição em ortostase, 12 apresentavam rupturas na raiz posterior $(25.5 \%)$.

A lesão junto à raiz posterior do menisco medial é considerada pela literatura como o principal fator associado à extrusão meniscal, contudo mesmo sobre a ação da carga corpórea por 1 minuto, a maioria dos meniscos com lesão na raiz posterior não apresentaram variação de posição ou maior grau de extrusão neste estudo.

Os indivíduos sintomáticos quanto à dor apresentaram valores de extrusão meniscal medial significativamente maiores que indivíduos sem dor (Figura 18). Wengeret al. (2012) encontraram resultados semelhantes em um estudo com RM, onde 43\% dos indivíduos com dor em sua amostra exibiam extrusão maior ou igual a $3 \mathrm{~mm}$, versus $23 \%$ dos indivíduos sem dor. Tais achados confirmam nossos resultados cuja média do grupo com dor foi 2,60 $\pm 1,95 \mathrm{~mm}$, enquanto a média do grupo sem dor não chegou nem a $1 \mathrm{~mm}$ (média: $0,63 \pm 1,02 \mathrm{~mm}$ ).

Como o aumento da idade pode acarretar em aumento de prevalência da osteoartrite, e esta altamente associada a leões meniscais, ao avaliar a influência da idade optamos por dividir os grupos quanto a presença ou não de OA. Além disso, em nossa amostra o grupo com presença de OA apresentou média de idade significativamente maior do que os indivíduos sem OA. Observou-se que o grupo sem OA apresentava uma correlação moderada, onde o aumento da idade foi acompanhado 
pelo aumento do valor mensurado de extrusão. Também foi visto uma correlação entre a idade e a extrusão do grupo com presença de OA, porém esta foi fraca. Tal fato nos permite afirmar que a idade se mostrou um importante fator associado à extrusão meniscal. Segundo Raiet al. (2013), a perda de genes da matriz extracelular com o envelhecimento indica diminuição da capacidade de reparo e aumento de alterações degenerativas no interior do menisco lesionado.

Com relação ao IMC, encontramos fraca correlação desta com a extensão da extrusão meniscal. Havivet al. (2015) encontraram uma correlação moderada entre o IMC e lesões condrais em indivíduos de meia idade (40-50 anos) com rupturas isoladas do menisco medial, porém em indivíduos com idade menor que 40 anos não foi encontrada esta correlação, enquanto que todas as lesões mais graves foram vistas em pacientes com mais de 50 anos. Já Feuchtet al. (2015), ao avaliarem fatores de risco para rupturas meniscais associadas à lesão do LCA, não encontraram influência do IMC sobre elas, mesmo em grupos cujo IMC foi maior que $30 \mathrm{~kg} / \mathrm{m}^{2}$. Tais achados levam a crer que fatores como idade e presença de OA possuem maior impacto na extrusão meniscal que o IMC.

Nosso trabalho apresenta algumas limitações. Para melhorar a reprodutibilidade quando comparamos a ultrassonografia e ressonância magnética nas mensurações da extrusão, decidimos incluir o osteófito quando presente, para medições em ambos os métodos. Isso pode, no entanto, levar à subestimar a extrusão meniscal. Além disso, apenas o corpo do menisco medial foi avaliado para mensuração de extrusão, pois a profundidade atingida pela ultrassonografia é insuficiente para visualizar todo o menisco, especialmente nos cornos anterior e posterior. Outra limitação potencial deste estudo é o fato de que nós incluímos em nossa amostra apenas os pacientes elegíveis para a RM de joelho, o que pode ser um viés de seleção. Por fim, visto que a maioria 
dos meniscos laterais em nossa amostra não mostraram extrusão nas avaliações realizadas, não foi possível realizar análises estatísticas em relação ao menisco lateral, e decidimos validar e reportar apenas medições para o menisco medial. Assim, não é certo que os nossos resultados também podem ser aplicados para o menisco lateral. 


\section{CONCLUSÃO}

Concluímos que houve variação na extrusão do menisco medial nas posições em decúbito e ortostase, aumentando a extrusão após carga pela posição ereta. Além disso, identificamos que a presença de extrusão $\geq 3 \mathrm{~mm}$ na ultrassonografia é indicativa de lesão meniscal.

A avaliação de extrusão meniscal medial com a ultrassonografia mostrou excelente desempenho diagnóstico para a detecção de extrusão meniscal quando comparada com ressonância magnética.

A presença de osteoartrite e a idade foram fatores associados com maior impacto para o aumento da extrusão meniscal que o IMC.

Com relação às diferenças entre os tipos de lesão, os maiores valores de extrusão foram encontrados nos meniscos macerados e rupturas complexas, seguidos pelas rupturas radiais da raiz posterior e flaps meniscais. 
1. Acebes C, Romero FI, Contreras MA, Mahillo I, Herrero-Beaumont G. Dynamic ultrasound assessment of medial meniscal subluxation in knee osteoarthritis. Rheumatology (Oxford). 2013;52(8):1443-7.

2. Allen DM, Li L, Crema MD, Marra MD, Guermazi A, Wyman BT, et al. The Relationship between Meniscal Tears and Meniscal Position.TherAdvMusculoskelet Dis. 2010;2(6):315-23.

3. Boxheimer L, Lutz AM, Treiber K, Goepfert K, Crook DW, Marincek B, et al. MR imaging of the knee: position related changes of the menisci in asymptomatic volunteers. Invest Radiol. 2004;39(5):254-63.

4. Boxheimer L, Lutz AM, Zanetti M, Treiber K, Labler L, Marincek B, et al. Characteristics of displaceable and nondisplaceable meniscal tears at kinematic MR imaging of the knee. Radiology. 2006;238(1):221-31.

5. Breitenseher MJ, Trattnig S, Dobrocky I, Kukla C, Nehrer S, Steiner E, et al. MR imaging of meniscal subluxation in the knee. ActaRadiol. 1997;38(5):876-9.

6. Brody JM, Lin HM, Hulstyn MJ, Tung GA. Lateral meniscus root tear and meniscus extrusion with anterior cruciate ligament tear.Radiology. 2006;239(3):805-10.

7. Burk DL, Dalinka MK, Kanal E, Schiebler ML, Cohen EK, Prorok RJ, et al. Meniscal and ganglion cysts of the knee: MR evaluation. AJR Am J Roentgenol. $1988 ; 150(2): 331-6$.

8. Cicuttini FM, Forbes A, Yuanyuan W, Rush G, Stuckey SL. Rate of knee cartilage loss after partial meniscectomy. J Rheumatol. 2002;29(9):1954-6.

9. Coral A, van Holsbeeck M, Adler RS. Imaging of meniscal cyst of the knee in three cases.Skeletal Radiol. 1989;18(6):451-5.

10. Crema MD, Guermazi A, Li L, Nogueira-Barbosa MH, Marra MD, Roemer FW, et al. The association of prevalent medial meniscal pathology with cartilage loss in the 
medial tibiofemoral compartment over a 2-year period. Osteoarthritis Cartilage. 2010;18(3):336-43.

11. Crema MD, Roemer FW, Felson DT, Englund M, Wang K, Jarraya M, et al. Factors associated with meniscal extrusion in knees with or at risk for osteoarthritis: the Multicenter Osteoarthritis study. Radiology. 2012;264(2):494-503.

12. De Smet AA. How I diagnose meniscal tears on knee MRI. AJR Am J Roentgenol. 2012;199(3):481-99.

13. De Smet AA, Norris MA, Yandow DR, Quintana FA, Graf BK, Keene JS. MR diagnosis of meniscal tears of the knee: importance of high signal in the meniscus that extends to the surface. AJR Am J Roentgenol. 1993;161(1):101-7.

14. Ding C, Martel-Pelletier J, Pelletier JP, Abram F, Raynauld JP, Cicuttini F, et al. Knee meniscal extrusion in a largely non-osteoarthritic cohort: association with greater loss of cartilage volume. Arthritis Res Ther. 2007;9(2):R21.

15. Englund M, Guermazi A, Gale D, Hunter DJ, Aliabadi P, Clancy M, et al. Incidental meniscal findings on knee MRI in middle-aged and elderly persons. $\mathrm{N}$ Engl $\mathrm{J}$ Med. 2008;359(11):1108-15.

16. Englund M, Guermazi A, Roemer FW, Aliabadi P, Yang M, Lewis CE, et al. Meniscal tear in knees without surgery and the development of radiographic osteoarthritis among middle-aged and elderly persons: The Multicenter Osteoarthritis Study. Arthritis Rheum. 2009;60(3):831-9.

17. Englund M, Roos EM, Lohmander LS. Impact of type of meniscal tear on radiographic and symptomatic knee osteoarthritis: a sixteen-year followup of meniscectomy with matched controls. Arthritis Rheum. 2003;48(8):2178-87.

18. Feucht MJ, Bigdon S, Bode G, Salzmann GM, Dovi-Akue D, Südkamp NP, et al. Associated tears of the lateral meniscus in anterior cruciate ligament injuries: risk 
factors for different tear patterns. J OrthopSurg Res. 2015;10:34.

19. Friedman L, Finlay K, Jurriaans E. Ultrasound of the knee. Skeletal Radiol. 2001;30(7):361-77.

20. Gale DR, Chaisson CE, Totterman SM, Schwartz RK, Gale ME, Felson D. Meniscal subluxation: association with osteoarthritis and joint space narrowing. Osteoarthritis Cartilage. 1999;7(6):526-32.

21. Harper KW, Helms CA, Lambert HS, Higgins LD. Radial meniscal tears: significance, incidence, and MR appearance. AJR Am J Roentgenol. 2005;185(6):142934.

22. Haviv B, Bronak S, Thein R. Correlation between body mass index and chondral lesions in isolated medial meniscus tears. Indian J Orthop. 2015;49(2):176-80.

23. Hunter DJ, Zhang YQ, Niu JB, Tu X, Amin S, Clancy M, et al. The association of meniscal pathologic changes with cartilage loss in symptomatic knee osteoarthritis.Arthritis Rheum. 2006;54(3):795-801.

24. Kawaguchi K, Enokida M, Otsuki R, Teshima R. Ultrasonographic evaluation of medial radial displacement of the medial meniscus in knee osteoarthritis. Arthritis Rheum. 2012;64(1):173-80.

25. Kellgren JH, Lawrence JS.Radiological assessment of osteo-arthrosis. Ann Rheum Dis. 1957;16(4):494-502.

26. Ko $\mathrm{CH}$, Chan KK, Peng HL. Sonographic imaging of meniscal subluxation in patients with radiographic knee osteoarthritis. J Formos Med Assoc. 2007;106(9):700-7. 27. Kumm J, Roemer FW, Guermazi A, Turkiewicz A, Englund M. Natural History of Intrameniscal Signal Intensity on Knee MR Images: Six Years of Data from the Osteoarthritis Initiative. Radiology.2015:142905.

28. Lo GH, Hunter DJ, Nevitt M, Lynch J, McAlindon TE, Group OI. Strong 
association of MRI meniscal derangement and bone marrow lesions in knee osteoarthritis: data from the osteoarthritis initiative. Osteoarthritis Cartilage. 2009;17(6):743-7.

29. Park GY, Kim JM, Lee SM, Lee MY. The value of ultrasonography in the detection of meniscal tears diagnosed by magnetic resonance imaging. Am J Phys Med Rehabil. 2008;87(1):14-20.

30. Peetrons P, Allaer D, Jeanmart L. Cysts of the semilunar cartilages of the knee: a new approach by ultrasound imaging. A study of six cases and review of the literature. $\mathrm{J}$ Ultrasound Med. 1990;9(6):333-7.

31. Petersen W, Tillmann B. Collagenous fibril texture of the human knee joint menisci. AnatEmbryol (Berl). 1998;197(4):317-24.

32. Rai MF, Patra D, Sandell LJ, Brophy RH. Transcriptome analysis of injured human meniscus reveals a distinct phenotype of meniscus degeneration with aging. Arthritis Rheum. 2013;65(8):2090-101.

33. Rath E, Richmond JC. The menisci: basic science and advances in treatment. Br J Sports Med. 2000;34(4):252-7.

34. Rennie WJ, Finlay DB. Meniscal extrusion in young athletes: associated knee joint abnormalities. AJR Am J Roentgenol. 2006;186(3):791-4.

35. Renström P, Johnson RJ. Anatomy and biomechanics of the menisci.Clin Sports Med. 1990;9(3):523-38.

36. Roemer FW, Zhang Y, Niu J, Lynch JA, Crema MD, Marra MD, et al. Tibiofemoral joint osteoarthritis: risk factors for MR-depicted fast cartilage loss over a 30-month period in the multicenter osteoarthritis study. Radiology. 2009;252(3):772-80.

37. Rosas HG. Magnetic resonance imaging of the meniscus.MagnReson Imaging Clin N Am. 2014;22(4):493-516. 
38. Seedhom BB, Dowson D, Wright V. Proceedings: Functions of the menisci. A preliminary study. Ann Rheum Dis. 1974;33(1):111.

39. Seymour R, Lloyd DC. Sonographic appearances of meniscal cysts.J Clin Ultrasound. 1998;26(1):15-20.

40. Stehling C, Souza RB, Hellio Le Graverand MP, Wyman BT, Li X, Majumdar S, et al. Loading of the knee during 3.0T MRI is associated with significantly increased medial meniscus extrusion in mild and moderate osteoarthritis. Eur $\mathrm{J}$ Radiol. 2012;81(8):1839-45.

41. Tibesku CO, Mastrokalos DS, Jagodzinski M, Pässler HH. [MRI evaluation of meniscal movement and deformation in vivo under load bearing condition].SportverletzSportschaden. 2004;18(2):68-75.

42. Vedi V, Williams A, Tennant SJ, Spouse E, Hunt DM, Gedroyc WM. Meniscal movement. An in-vivo study using dynamic MRI. J Bone Joint Surg Br. 1999;81(1):3741.

43. Verdonk P, Depaepe Y, Desmyter S, De Muynck M, Almqvist KF, Verstraete K, et al. Normal and transplanted lateral knee menisci: evaluation of extrusion using magnetic resonance imaging and ultrasound. Knee Surg Sports TraumatolArthrosc. 2004;12(5):411-9.

44. Wang Y, Wluka AE, Pelletier JP, Martel-Pelletier J, Abram F, Ding C, et al. Meniscal extrusion predicts increases in subchondral bone marrow lesions and bone cysts and expansion of subchondral bone in osteoarthritic knees. Rheumatology (Oxford). 2010;49(5):997-1004.

45. Wareluk P, Szopinski KT. Value of modern sonography in the assessment of meniscal lesions. Eur J Radiol. 2012;81(9):2366-9.

46. Wenger A, Englund M, Wirth W, Hudelmaier M, Kwoh K, Eckstein F, et al. 
Relationship of 3D meniscal morphology and position with knee pain in subjects with knee osteoarthritis: a pilot study. EurRadiol. 2012;22(1):211-20.

47. Woolf AD, Pfleger B. Burden of major musculoskeletal conditions. Bull World Health Organ. 2003;81(9):646-56.

48. Woolf AD, Pfleger B. Burden of major musculoskeletal conditions. Bull World Health Organ. 2003;81(9):646-56. 
ANEXO A - Carta de Aprovação do Comitê de Ética e Pesquisa

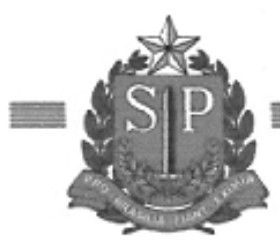

HOSPITAL DAS CLINICAS DA FACULDADE DE MEDICINA DE RIBEIRÃO PRETO DA UNIVERSIDADE DE SÄO PAULO

wuw.herp.usp.br

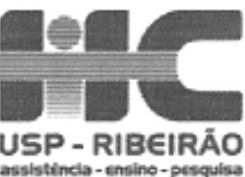

USP - RIBEIRÃO

Ribeirão Preto, 03 de agosto de 2011

Oficio $\mathrm{n}^{\circ} 2804 / 2011$

CEP/MGV

Prezados Senhores,

O trabalho intitulado "AVALIAÇÃO DOS FATOES ASSOCIADOS À EXTRUSÃo MENISCAL EM AMBOS OS COMPARTIMENTOS FÊMORO-TIBIAIS NA PRESENÇA E NA AUSÊNCIA DA CARGA CORPÓREA" foi analisado pelo Comitê de Ética em Pesquisa, em sua $329^{a}$ Reunião Ordinária realizada em 01/08/2011 e enquadrado na categoria: APROVADO, bem como o Termo de Consentimento Livre e Esclarecido, de acordo com o Processo HCRP n ${ }^{\circ} 8989 / 2011$.

Este Comite segue integralmente a Conferencia Internacional de Harmonizaçāo de Boas Práticas Clínicas (IGH-GCP), bem como a Resoluçāo $n^{\circ}$ 196/96 CNS/MS.

Lembramos que devem ser apresentados a este CEP, o Relatório Parcial e o Relatório Final da pesquisa.

De acordo com Carta Circular $n^{\circ} 003 / 2011 / C O N E P / C N S$ datada de 21 de março de 2011, o sujeito de pesquisa ou seu representante, quando for o caso, deverá rubricar todas as folhas do Termo de Consentimento Livre e Esclarecido - TCLE - apondo sua assinatura na última página do referido Termo; o pesquisador responsável deverá da mesma forma, rubricar todas as folhas do Termo de Consentimento Livre e Esclarecido TCLE - apondo sua assinatura na última página do referido Termo.

Atenciosamente,

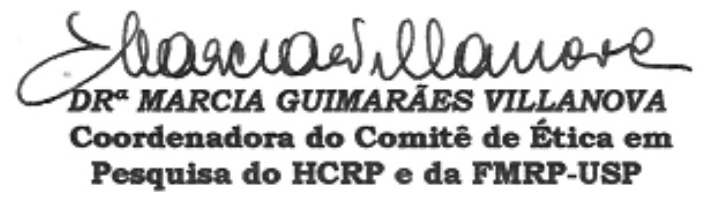


ANEXO B - Termo de Consentimento Livre e Esclarecido

TERMO DE CONSENTIMENTO LIVRE E ESCLARECIDO

Título do Projeto: FATORES DIRETOS E INDIRETOS ASSOCIADOS A EXTRUSÃO MENISCAL NO COMPARTIMENTO FÊMORO-TIBIAL MEDIAL NA PRESENÇA E AUSÊNCIA DA CARGA CORPÓREA.

Pesquisadores Responsáveis: Everaldo Gregio Junior, Michel Daoud Crema e Prof. Dr. Marcello Henrique Nogueira - Barbosa

Instituição a que pertence o Pesquisador Responsável: Hospital das Clínicas da Faculdade de Medicina de Ribeirão Preto, Universidade de São Paulo, Ribeirão PretoSP.

Telefone para contato: (16) 3602-2640 (16) 99749-2266

Nome

do

voluntário:

Idade:

anos

R.G.

Responsável

legal

(quando

for

0

caso):

R.G. Responsável legal: 


\section{Justificativas e objetivos:}

O senhor(a) veio para a realização de uma ressonância magnética do joelho, sendo este exame fundamental para a avaliação das estruturas internas do joelho antes de um eventual procedimento cirúrgico, chamado artroscopia do joelho. 0 exame de ressonância magnética faz parte da rotina de avaliação pré-operatória, e será realizada de qualquer forma independentemente da pequisa atual. Estamos convidando o senhor(a) para participar de um estudo com objetivo de avaliar os fatores e comorbidades diretamente relacionadas a lesão meniscal..

\section{Descrição dos métodos:}

O senhor(a), se voluntário deste estudo, terá seu joelho examinado por ressonância magnética antes de eventual artroscopia do mesmo joelho conforme pedido pelo seu medico. Além da ressonância de rotina, serão realizados no mesmo local e período dois exames de imagen complementares, sendo estes; ultra-son em posição ortostática e decúbito e raio-x dos joelhos em incidência única $A P$, e também a coleta de dados pessoais como peso e altura para o calculo do índice de massa corpórea sem que haja qualquer custo financeiro.

\section{Desconfortos e riscos associados:}

Durante o exame de ressonância magnética o paciente ouvirá um barulho parecido com batidas que ocorrem em intervalos regulares, o que pode ocasionar certo desconforto. Esse barulho é normal e esperado no caso do exame de ressonância e a sua presença significa que as imagens estão sendo feitas. O nível do barulho não é prejudicial a sua audição. Um protetor contra ruídos para os ouvidos será oferecido para minimizar este efeito. Algumas pessoas se incomodam em ficar dentro do aparelho de ressonância ou até mesmo não conseguem ficar lá dentro porque tem dificuldade em ficar em lugares fechados ou que consideram apertados. Estas pessoas têm claustrofobia e se o paciente tiver este tipo de problema não será feito o exame e isto não vai prejudicar o tratamento deste paciente. O exame de ressonância magnética pode trazer risco para algumas pessoas portadoras de alguns tipos de material metálico, sendo que um questionário de segurança será oferecido, preenchido e assinado pelosenhor(a) antes da realização da ressonância magnética. Não será administrado nenhum produto de contraste paramagnético para a realização deste protocolo. 
Liberdade de participação:

A participação neste estudo depende plenamente da sua autorização. É seu direito interromper a sua participação a qualquer momento, inclusive após o inicio do exame de ressonância magnética, sem que isso incorra em qualquer penalidade ou prejuízo contra o senhor(a). Os pesquisadores deste estudo têm o direito de não utilizar os seus dados neste experimento no caso de abandono da pesquisa, exame incompleto ou a sua não colaboração durante o período de exame.

\section{Benefícios esperados (para o voluntário e/ou para a comunidade):}

Eu entendo que não existe nenhum tipo de benefício econômico, seguro de saúde ou de vida que possa me beneficiar em função da minha participação neste estudo. As alterações que ocasionalmente podem ser identificados nos exames complementares realizados pelo estudo, serão avaliadas em conjunto e descritas no relatório medico para melhor aproximação diagnostica.

\section{Sigilo de identidade:}

As informações obtidas nessa pesquisa não serão de maneira alguma associadas à sua identidade. E não poderão ser consultadas por pessoas leigas sem sua autorização oficial. Estas informações poderão ser utilizadas para fins estatísticos ou científicos, desde que fiquem resguardados a sua privacidade e seu anonimato.

\section{Valores e formas de ressarcimento:}

A realização das técnicas adicionais em ressonância magnética será realizado sem ônus ao senhor(a). O senhor(a) não receberá nenhuma compensação financeira ao aceitar ser voluntário(a) deste estudo. 
$\mathrm{Eu}$,

$R G$

no

declaro ter sido informado e concordo em participar, como

voluntário, do projeto de pesquisa acima descrito.Os responsáveis pelo estudo me explicaram os riscos envolvidos, a necessidade da pesquisa e se prontificaram a responder todas as dúvidas sobre a pesquisa. Eu aceitei participar deste estudo de livre e espontânea vontade. Entendo que é meu direito manter uma cópia deste consentimento

Ou

$\mathrm{Eu}$, RG $\quad n^{\circ}$ responsável legal por RG $\mathrm{n}^{\circ}$

declaro ter sido informado e concordo com a sua participação, como voluntário, no projeto de pesquisa acima descrito.

Ribeirão Preto, de de 


\section{ANEXO C - Índice WOMAC para Osteoartrose \\ ÍNDICE WOMAC PARA OSTEOARTROSE}

Nome:

Data

avaliação:

ID:

As perguntas a seguir se referem à INTENSIDADE DA DOR que você está atualmente sentindo devido a artrite de seu joelho. Para cada situação, por favor, coloque a intensidade da dor que sentiu nas últimas 72 horas ( 3 dias)

Pergunta: Qual a intensidade da sua dor?

\begin{tabular}{|c|c|c|c|c|}
\hline \multicolumn{5}{|c|}{ 1-Caminhando em um lugar plano. } \\
\hline Nenhuma $\square$ & Pouca $\square$ & Moderada & Intensa & Muito intensa \\
\hline \multicolumn{5}{|c|}{ 2- Subindo ou descendo escadas. } \\
\hline Nenhuma & Pouca & Moderada & Intensa & Muito intensa \\
\hline \multicolumn{5}{|c|}{ 3- A noite deitado na cama. } \\
\hline Nenhuma & Pouca [ & Moderada & Intensa & Muito intensa \\
\hline \multicolumn{5}{|c|}{ 4-Sentando-se ou deitando-se. } \\
\hline Nenhuma & Pouca & Moderada & Intensa & Muito intensa \\
\hline \multicolumn{5}{|c|}{ 5. Ficando em pé. } \\
\hline Nenhuma & Pouca $\square$ & Moderada & Intensa & Muito intensa \\
\hline
\end{tabular}

\section{TOTAL:}

As perguntas a seguir se referem a intensidade de RIGIDEZ nas junta (não dor), que você está atualmente sentindo devido a artrite em seu joelho nas últimas 72 horas. Rigidez é uma sensação de restrição ou dificuldade para movimentar suas juntas. 
1- Qual é a intensidade de sua rigidez logo após acordar de manhã?

Nenhuma $\square \quad$ Pouca $\square$ Moderada $\square$ Intensa $\square$ Muito intensa

2- Qual é a intensidade de sua rigidez após se sentar, se deitar ou repousar no decorrer do dia?

Nenhuma $\square$ Pouca $\square$ Moderada $\square$ Intensa $\square$ Muito intensa

\section{TOTAL:}

As perguntas a seguir se referem a sua ATIVIDADE FÍSICA. Nós chamamos atividade física, sua capacidade de se movimentar e cuidar de você mesmo(a). Para cada uma das atividades a seguir, por favor, indique o grau de dificuldade que você está tendo devido à artrite em seu joelho durante as últimas 72 horas.

Pergunta: Qual o grau de dificuldade que você tem ao:

\begin{tabular}{|c|c|c|c|c|}
\hline \multicolumn{5}{|c|}{1 - Descer escadas. } \\
\hline Nenhuma & Pouca & Moderada & Intensa & Muito intensa \\
\hline \multicolumn{5}{|c|}{ 2- Subir escadas. } \\
\hline Nenhuma & Pouca & Moderada & Intensa & Muito intensa \\
\hline \multicolumn{5}{|c|}{ 3- Levantar-se estando sentada. } \\
\hline Nenhuma & Pouca & Moderada & Intensa & Muito intensa \\
\hline \multicolumn{5}{|c|}{ 4- Ficar em pé. } \\
\hline Nenhuma & Pouca & Moderada & Intensa & Muito intensa \\
\hline
\end{tabular}

\begin{tabular}{|c|c|c|c|c|}
\hline \multicolumn{5}{|c|}{ 5- Abaixar-se para pegar algo. } \\
\hline Nenhuma & Pouca & Moderada & Intensa & Muito intensa \\
\hline \multicolumn{5}{|c|}{ 6- Andar no plano. } \\
\hline Nenhuma & Pouca & Moderada & Intensa & Muito intensa \\
\hline \multicolumn{5}{|c|}{ 7- Entrar e sair do carro. } \\
\hline Nenhuma & Pouca & Moderada & Intensa & Muito intensa \\
\hline \multicolumn{5}{|c|}{ 8- Ir fazer compras. } \\
\hline Nenhuma & Pouca & Moderada & Intensa & Muito intensa \\
\hline \multicolumn{5}{|c|}{ 9- Colocar meias. } \\
\hline Nenhuma & Pouca & Moderada & Intensa & Muito intensa \\
\hline
\end{tabular}
10- Levantar-se da cama. 


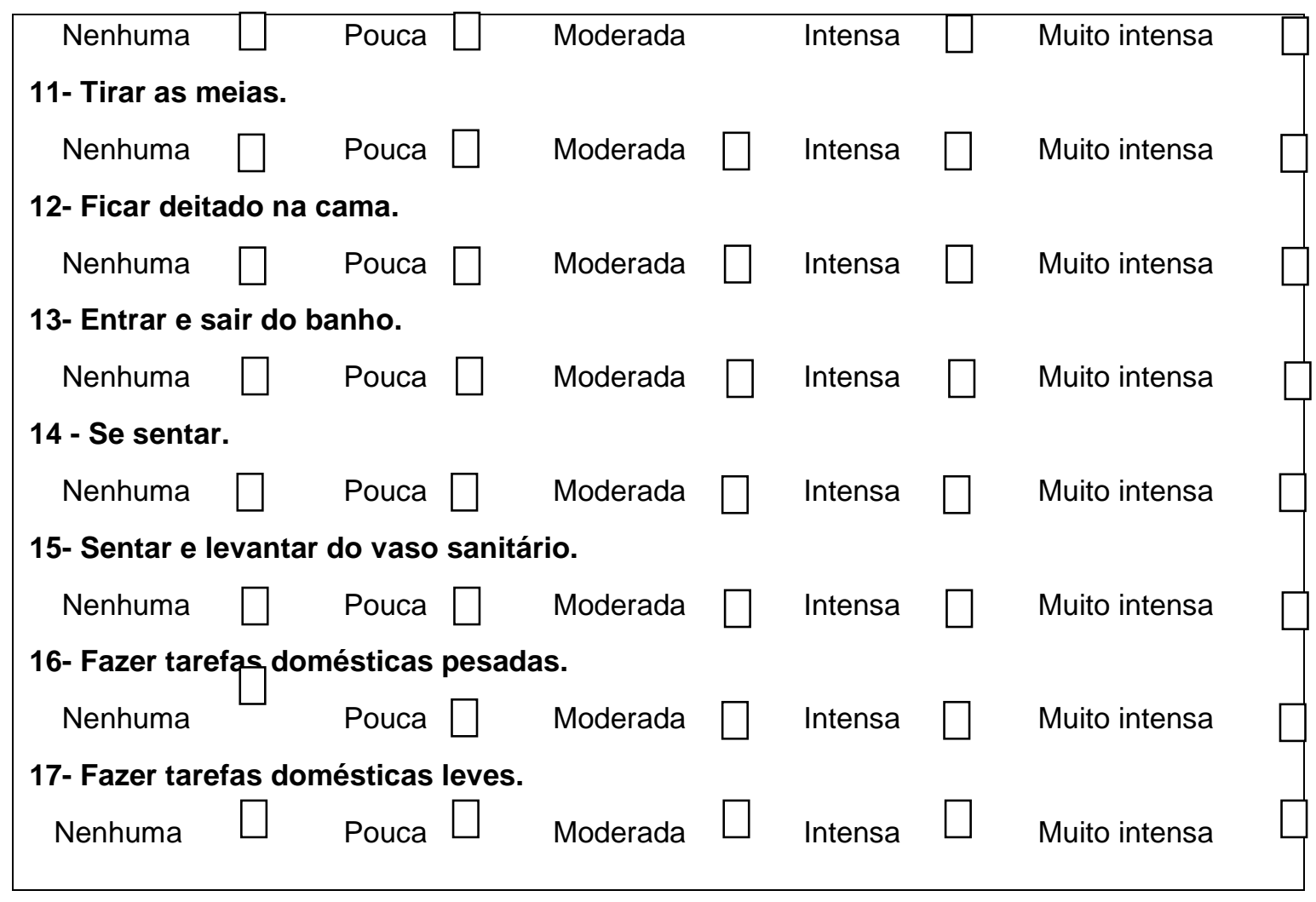

TOTAL:

OBRIGADO POR COMPLETAR ESTE QUESTIONÁRIO 


\section{ANEXO D - Escala Visual Analógica}

ESCALA VISUAL ANALÓGICA - EVA

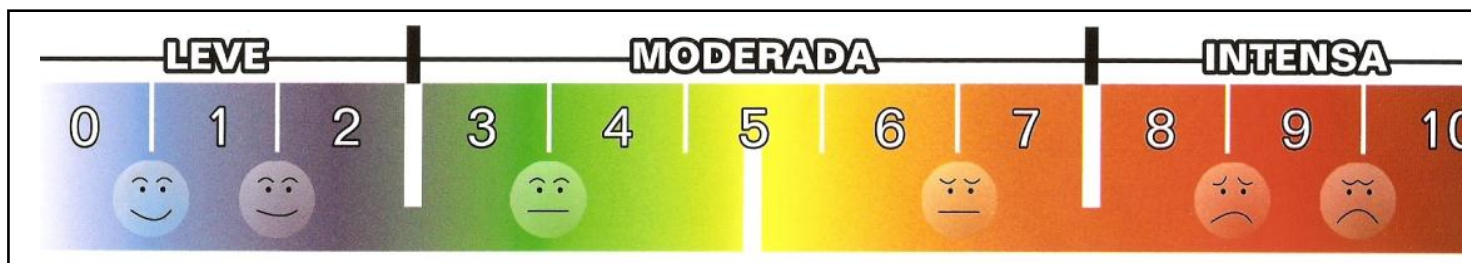

ESCALA VISUAL ANALÓGICA - EVA

A Escala Visual Analógica - EVA consiste em auxiliar na aferição da intensidade da dor no paciente, é um instrumento importante para verificarmos a evolução do paciente durante o tratamento e mesmo a cada atendimento, de maneira mais fidedigna. Também é útil para podermos analisar se o tratamento está sendo efetivo, quais procedimentos têm surtido melhores resultados, assim como se há alguma deficiência no tratamento, de acordo com o grau de melhora ou piora da dor.

A EVA pode ser utilizada no início e no final de cada atendimento, registrando o resultado sempre na evolução. Para utilizar a EVA o atendente deve questionar o paciente quanto ao seu grau de dor sendo que $\mathbf{0}$ significa ausência total de dor e 10 o nivel de dor máxima suportável pelo paciente.

Dicas sobre como interrogar o paciente:

- Você tem dor?

- Como você classifica sua dor? (deixe ele falar livremente, faça observações na pasta sobre o Questione-0: que ele falar)

a) Se não tiver dor, a classificação é zero.

b) Se a dor for moderada, seu nível de referência é cinco.

c) Se for intensa, seu nível de referência é dez.

OBS.: Procure estabelecer variações de melhora e piora na escala acima tomando cuidado para não sugestionar o paciente. 


\section{ANEXO E - Artigo publicado no periódico AJR}

\section{Ultrasound Assessment of Medial Meniscal Extrusion: A Validation Study Using MRI as Reference Standard}

Marcello H. Nogueira-Barbosa Everaldo Gregio-Junior

Mario M. Lorenzato ${ }^{1}$

Ali Guermazi ${ }^{2}$

Frank W. Roemer 2,3

Francisco A. Chagas-Neto $0^{1,5}$

Michel D. Crema 2,4

Keywords: extrusion, knee, meniscus, MRI, ultrasound

DOI:10.2214/A.JR.14.12522

Received January 11, 2014; accepted after revision June 27, 2014

M. D. Crema and F. W. Roemer are stockholders of Boston Imaging Core Laboratory (BICL). A. Guermazi is president Genzyme, Novartis, Stryker, and AstraZeneca.

Coordenaçāo de Aperfeiçoamento de Pessoal de Nivel

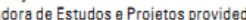
financial support for this study.

'Division of Radiology of the Internal Medicine Department Ribeirao Preto School of Medicine-University of Sao Paulo, Ribeirao Preto, Brazil.

${ }^{2}$ Quantitative Imaging Center, Department of Radiologr Boston University School of Medicine, 820 Herrison Ave, FGH BId 3rd F1, Boston, MA 02118 Address correspon. FoH Bly

${ }^{3}$ Department of Radiology, University of Erlangen, Erlangen, Germany.

${ }^{4}$ Department of Radiology, Hospital do Coração and Teleimagem, São Paulo, SP, Brazi

"Radiology Division, Christus University Center, Fortaleza, CE, Brazil.

AJR2015; 204:584-588

$0361-803 X / 15 / 2043-584$

(ㄱ) American Roentgen Ray Society
OBJECTIVE. The purpose of this article is to validate both semiquantitative and quantitative ultrasound assessment of medial meniscal extrusion using MRI assessment as the reference standard.

SUBJECTS AND METHODS. Ninety-three consecutive patients with chronic knee pain referred for knee MRI were evaluated by ultrasound and MRI on the same day. Two musculoskeletal radiologists assessed meniscal extrusion on ultrasound and MRI separately and independently and graded it semiquantitatively as follows: $0(<2 \mathrm{~mm}), 1(\geq 2 \mathrm{~mm}$ and $<4$ $\mathrm{mm})$, and $2(\geq 4 \mathrm{~mm})$. Agreement between the ultrasound and MRI evaluations was determined using weighted kappa statistics. Intraclass correlation coefficients were used to evaluate agreement using the absolute values of extrusion (quantitative assessment). We further evaluated the diagnostic performance of ultrasound for the detection of medial meniscal extrusion using MRI as the reference standard.

RESULTS. For semiquantitative grading, agreement between ultrasound and MRI was moderate for reader I $(\kappa=0.57)$ and substantial for reader $2(\kappa=0.64)$. Substantial agreement was found for both readers (intraclass correlation coefficients, 0.73 and 0.70 ) when comparing quantitative assessment of meniscal extrusion between ultrasound and MRI. Ultrasound showed excellent sensitivity ( $95 \%$ and $96 \%$ for each reader) and good specificity ( $82 \%$ and $70 \%$ for each reader) in the detection of meniscal extrusion.

CONCLUSION. Ultrasound assessment of meniscal extrusion is reliable and can be used for both quantitative and semiquantitative assessment, exhibiting excellent diagnostic performance for the detection of meniscal extrusion compared with MRI.

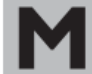
echanical dysfunction of the meniscus may predispose to articular cartilage and subchondral bone damage, thus contributing to the progression of knee osteoarthritis [16]. Meniscal extrusion (also known as subluxation) is referred to as external meniscal displacement with regard to the external edge or aspect of the tibiofemoral compartment. It may impair meniscal function and, despite its strong association with concomitant meniscal tears or loss of meniscal substance [7-9], it is thought to be an independent predictor of tibiofemoral cartilage loss [7, 10-13]. Most studies that assessed factors associated with meniscal extrusion, such as meniscal tears, meniscal maceration, cartilage damage, and knee malalignment, were cross-sectional studies, and it is difficult to determine whether these factors represent causes of extrusion [7-9]. Imaging techniques for the assessment of meniscal extrusion can provide useful in-

formation for understanding the causes and consequences of meniscal extrusion and also the effects of extrusion on the tibiofemoral compartments of the knee.

MRI is considered the method of choic for noninvasive detection of meniscal abnormalities, including meniscal extrusion, and has been used with semiquantitative and quantitative assessments [7, 12-14]. Routine MRI examinations do not evaluate the meniscus under axial loading (weight bearing), and this is sometimes considered a drawback of the method when the assessment of meniscal position (extrusion) is relevant. Ultrasound is widely available and has also been used for the assessment of meniscal extrusion [15-18]. Ultrasound also may offer a relatively simple way to assess meniscal extrusion with the patient both supine (at rest) -17]. Before applying ultrasound to the assessment of meniscal extrusion in clinical practice and 
Ultrasound of Medial Meniscal Extrusion

research, however, it is of the utmost importance to validate the method against an accepted reference standard.

The aim of this study was to provide just such a validation of ultrasound for the assessment of medial meniscal extrusion, using MRI assessment as the reference standard.

\section{Subjects and Methods}

\section{Participants}

We included patients with chronic knee pain who underwent an MRI of the knee in our department and who also consented to an ultrasound examination of the same knee on the same day (inclusion criteria). The exclusion criteria were previous surgery to the knee assessed (partial or total meniscectomy) and previous fracture of the knee assessed. The study cohort consisted of 93 consecutive patients, one knee per patient ( 50 men and 43 women; mean age $[ \pm S D], 41.5 \pm 13.8$ years; age range, 18-70 years; mean body mass index [weight in kilograms divided by the square of height in meters], $28.7 \pm 5.8$; range, 20-47). After inclusion (and further MRI and ultrasound assessments), no patient was excluded. For all 93 knees, the body of the medial meniscus was depicted in both ultrasound and MRI, and extrusion measurements could be performed. Knees were included in the study regardless of the presence of other abnormalities in the knee joint. The study protocol was approved by the local institutional review board, and we obtained signed informed consent from all the patients.

\section{Ultrasound Acquisition}

Ultrasound assessment of the knee was performed on the same day for all patients and just before the MRI. Ultrasound was performed using a scanner (Logiq $e$, GE Healthcare) with a 5- to $12-\mathrm{MHz}$ linear probe. Patients were supine, with the knee fully extended. Ultrasound images were acquired at the medial aspect of the knee using longitudinal sections parallel to the medial collateral ligament. A musculoskeletal radiologist with 3 years of experience with musculoskeletal ultrasound performed all of the ultrasound assessments of the medial menisci, choosing and saving the longitudinal sections for meniscal extrusion measurements. To ensure that the extrusion would be measured at exactly the same longitudinal (coronal) section on MRI as on ultrasound, two skin markers were placed at the level of the maximum extrusion as determined by the ultrasound examination (Fig. 1)

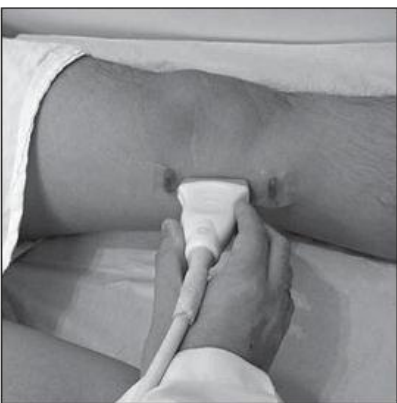

Fig. 1-28-year-old male volunteer. Ultrasoun evaluation of medial meniscal extrusion was performed in longitudinal axis with patient in supine of two skin markers where ulted. Note placem were documented for further meniscal extrusion assessment.

8-channel knee coil (Sense, Philips Healthcare) The routine MRI was performed with knees fully extended and included four sequences: first, sagittal intermediate-weighted turbo spin-echo (TSE) fat-suppressed (TR/TE, 2342/50; matrix, $224 \times$ 176; FOV, $16 \times 16 \mathrm{~cm}$; $4-\mathrm{mm}$ slice thickness number of excitations, 4 ; echo-train length, 14 bandwidth, $395 \mathrm{~Hz} /$ pixel; acquisition time, 2 min utes 43 seconds); second, coronal intermediate weighted TSE fat-suppressed (TR/TE, 2342/50; matrix, $224 \times 176$; FOV, $16 \times 16 \mathrm{~cm}$; 4-mm slic thickness; number of excitations, 4 ; echo-trai length, 14; bandwidth, $386 \mathrm{~Hz} /$ pixel; acquisitio time 2 minutes 30 seconds); third, axial intermediate-weighted TSE fat-suppressed (TR/TE, $3045 / 50$; matrix, $224 \times 176$; FOV, $16 \times 16 \mathrm{~cm}$; $4-\mathrm{mm}$ slice thickness; number of excitations, 4 echo-train length, 14; bandwidth, $429 \mathrm{~Hz} /$ pixel; acquisition time 3 minutes); and fourth, sagitta Tl-weighted spin-echo (TR/TE, 532/20; matrix, $292 \times 196$; FOV, $20 \times 20 \mathrm{~cm}$; $4-\mathrm{mm}$ slice thickness; number of excitations, 3 ; echo-train length, 6; bandwidth, $349.5 \mathrm{~Hz} /$ pixel; acquisition time minute $4 \mathrm{l}$ seconds).

On MRI, the coronal intermediate-weighte TSE fat-suppressed sequence was used to evalute extrusion, using the slice that included both of the skin markers that were placed during the ultra sound examination (Fig. 2).

Ultrasound and MRI Assessments of Media Meniscal Extrusion

One month after all the examinations were completed, two musculoskeletal radiologists with

MRI Acquisition

All knees were imaged with the same 1.5-T MRI unit (Achieva, Philips Healthcare) using an

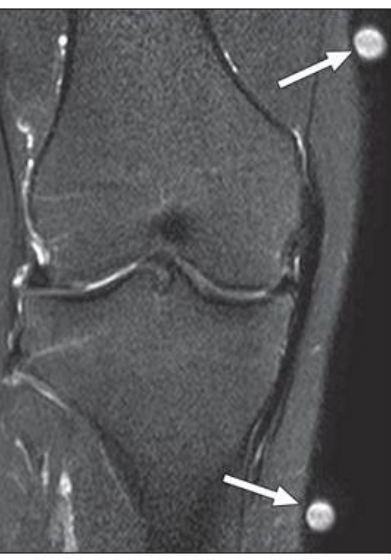

Fig. 2-45-year-old man with chronic knee pain. Coronal intermediate-weighted turbo spin-echo fatsuppressed MRI shows both skin markers (arrows) placed during ultrasound. This was reference image
for medial meniscal extrusion assessment on MRI.

ultrasound sections. The readers were blinded to clinical and MRI data. A line parallel to the longitudinal (craniocaudal) axis of ultrasound image was drawn at the external edge of the medial tibial plateau and served as a reference for extrusion measurements ( Fig 3). One month after the ultrasound measurements were performed, the same readers, again blinded to clinical and ultrasound data, independently assessed medial meniscal extrusion using the coronal intermediate-weighted TSE fat-suppressed images that included both skin markers. Again, a longitudinal line paralle to the craniocaudal axis of the coronal MR image and passing by the external edge of the medial tib. ial plateau was drawn and served as a reference for extrusion measurements [7, 12] (Fig. 3). For both ultrasound and MRI assessments, the extrusion measurement was considered as the perpendicular distance between the reference longitudinal (coronal) line drawn and the outer margin of the body of the meniscus (Fig. 3). Meniscal extrusion was measured to the nearest millimeter in both imaging methods (absolute values). To enhance reprowas systematically performed in ultrasound and MRI without excluding tibial osteophytes whenever these were present.

All measurements were performed using workstation (eFilm version 340. Merge Healthcare). Measurements of extrusion were made on screen with calipers. Both readers set the measurements to the nearest millimeter. Because both 
Nogueira-Barbosa et al.

semiquantitative and quantitative approaches of measurement were previously applied in the literature for meniscal extrusion assessment [7, 12 14], we decided to validate both approaches, Semiquantitative assessment refers to the distribution of meniscal extrusion according to an ordinal grading system proposed to determine its severity or extent, and this was defined in the current study as follows: grade $0(<2 \mathrm{~mm})$, grade $\mathrm{l}(\geq 2 \mathrm{~mm}$ and $<4 \mathrm{~mm})$, and grade $2(\geq 4 \mathrm{~mm})$. The quantitative assessment performed in this study referred simply to the absolute and direct measurements of meniscal extrusion performed (in millimeters). Ultrasound and MR images of patients included were read twice by both readers for intraobserver reliability assessment, within a time frame of at least 1 month.

Analytic Approach

The results of the semiquantitative assessments of meniscal extrusion (grades $0-2$ ) on ultrasound and MRI were compared using weighted kappa statistics. Intra- and interobserver reliability for ultrasound and MRI extrusion grades were assessed using kappa statistics. Intraclass correla tion coefficients were obtained to determine the agreement between assessments on ultrasoun and MRI using the absolute values of measurements of meniscal extrusion (quantitative assessment). Finally, the diagnostic performance of ultrasound for the detection of meniscal extrusion, defined as grade I or higher, was evaluated using MRI as the reference standard.

\section{Results}

The comparison between semiquantitative assessments of medial meniscal extrusion on ultrasound and MRI by the same reader showed moderate-to-substantial agreement (Table 1). Interobserver agreement was substantial for MRI and almost perfect for ultrasound (Table 2). The distribution of semiquantitative grades assigned for each reader in each imaging method (ultrasound and MRI) is also displayed in Tables I and 2. For reader 1 , intraobserver agreement was substantial for MRI $(\kappa=0.70 ; 95 \% \mathrm{CI}$, $0.45-0.94)$ and almost perfect for ultrasound $(\kappa=0.99 ; 95 \% \mathrm{CI}, 0.76-1.0)$. For reader 2 , intraobserver agreement was substantial for MRI ( $\mathrm{K}=0.68 ; 95 \% \mathrm{Cl}, 0.42-0.93)$ and almost perfect for ultrasound $(\kappa=0.91$; $95 \%$ CI, 0.66-1.0).

For quantitative measurements, the average $( \pm S D)$ meniscal extrusion for reader was $1.4 \pm 1.5 \mathrm{~mm}$ on ultrasound and $1.4 \pm 1.4$ $\mathrm{mm}$ on MRI. The average meniscal extrusion for reader 2 was $1.4 \pm 1.5 \mathrm{~mm}$ on ultrasound and $1.3 \pm 1.4 \mathrm{~mm}$ on MRI. When using abso-

TABLE I: Comparison (Agreement) of Medial Meniscal Extrusion Grades Between Ultrasound and MRI for Each Reader

\begin{tabular}{l|c|c|c|c}
\hline \multirow{2}{*}{ Reader, MRI Grade } & \multicolumn{3}{|c|}{ Ultrasound } & \multirow{2}{*}{ Total } \\
\cline { 2 - 4 } & Grade 0 & Grade 1 & Grade 2 & \\
\hline Reader 1 & & & & 55 \\
Grade 0 & 46 & 8 & 1 & 28 \\
Grade 1 & 11 & 14 & 3 & 10 \\
Grade 2 & 0 & 3 & 7 & 93 \\
\hline Total & 57 & 25 & 11 & 55 \\
\hline Reader 2 & & & & 27 \\
Grade 0 & 47 & 7 & 1 & 11 \\
Grade 1 & 10 & 15 & 2 & 93 \\
Grade 2 & 0 & 2 & 9 & 12 \\
\hline Total & 57 & 24 & & \\
\hline
\end{tabular}

Note-Data are number of knees. Weighted $\mathrm{K}=0.57(95 \% \mathrm{Cl}, 0.41-0.73$

TABLE 2: Interobserver Agreement for Medial Meniscal Extrusion Assessed in Each Imaging Method

\begin{tabular}{l|c|c|c|c}
\hline \multirow{2}{*}{ Reader 1 } & \multicolumn{3}{|c|}{ Reader 2 } & \multirow{2}{*}{ Total } \\
\cline { 2 - 4 } & Grade 0 & Grade 1 & Grade 2 & \\
\hline MRI $^{\text {s }}$ & & & & 55 \\
Grade 0 & 47 & 8 & 0 & 27 \\
Grade 1 & 8 & 18 & 1 & 11 \\
Grade 2 & 0 & 2 & 9 & 93 \\
\hline Total & 55 & 28 & 10 & \\
\hline Ultrasound & & & & 57 \\
Grade 0 & 57 & 0 & 0 & 24 \\
Grade 1 & 0 & 24 & 0 & 12 \\
Grade 2 & 0 & 1 & 11 & 93 \\
\hline Total & 57 & 25 & 11 & \\
\hline
\end{tabular}

Note-Data are number of knees.

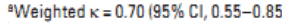

bWeighted $\mathrm{K}=0.98(95 \% \mathrm{Cl}, 0.94-1.00)$

TABLE 3: Diagnostic Performance of Ultrasound in the Detection of Media Meniscal Extrusion Compared With MRI

\begin{tabular}{|c|c|c|c|c|c|c|}
\hline \multirow[b]{2}{*}{ Ultrasound } & \multicolumn{3}{|c|}{ MRI } & \multirow{2}{*}{$\begin{array}{c}\text { Sensitivity } \\
(\%)\end{array}$} & \multirow{2}{*}{$\begin{array}{c}\text { Specificity } \\
(\%)\end{array}$} & \multirow{2}{*}{$\begin{array}{c}\text { Accuracy } \\
(\%)\end{array}$} \\
\hline & Normal & Abnormal & Total & & & \\
\hline Reader 1 & & & & 95 & 70 & 92 \\
\hline Normal & 79 & 3 & 82 & & & \\
\hline Abnormal & 4 & 7 & 11 & & & \\
\hline Total & 83 & 10 & 93 & & & \\
\hline Reader 2 & & & & 96 & 82 & 95 \\
\hline Normal & 79 & 2 & 81 & & & \\
\hline Abnormal & 3 & 9 & 12 & & & \\
\hline Total & 82 & 11 & 93 & & & \\
\hline
\end{tabular}

Note-Data are number of knees. 
lute medial meniscal extrusion values (quantitative assessment) measured by the same reader, the comparison between ultrasound and MRI assessments showed substantial agreement for both readers, with intraclass correlation coefficients of $0.70(95 \% \mathrm{CI}$, $0.58-0.79$ ) and 0.73 (95\% CI, 0.58-0.79).

Ultrasound showed excellent diagnostic performance for the detection of medial meniscal extrusion when taking MRI as the reference, with accuracies of $92 \%$ and $95 \%$ for each respective reader (Table 3 ).

\section{Discussion}

We tested the reliability and diagnostic performance of ultrasound for the assessment of medial meniscal extrusion and compared the results to the MRI assessment as a reference standard. The semiquantitative and quantitative assessments of meniscal extrusion based on ultrasound showed moderateto-substantial agreement, as well as excellent diagnostic performance in the detection of extrusion. To our knowledge, this is the first study to directly compare ultrasound assessment of meniscal extrusion with a reference standard such as MRI. Although previous studies have already applied ultrasound to the assessment of meniscal extrusion [1518], the use of ultrasound to assess meniscal extrusion has not been validated on MRI, as it must be before it can be widely applied in clinical practice and research.

Ultrasound is an operator-dependent technique, and when there is not enough contrast in echogenicity between anatomic structures, it can be difficult to accurately delineate the meniscus. This is true especially in cases of moderate-to-advanced degenerative changes in the medial tibiofemoral compartment, with changes affecting not only the medial meniscus but also the adjacent soft

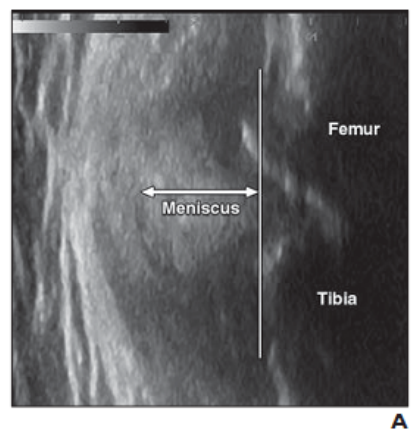

tissues, such as the medial collateral ligament and synovium. Furthermore, althoug there is some evidence that ultrasound can accurately diagnose meniscal tears [19], the possible use of ultrasound for meniscal tears will still require validation.

One of the major advantages of MRI is that the contrast and signal can be manipulated to highlight different tissues around the joint, making MRI widely accepted as the imaging method of reference in the assessment of meniscal abnormalities, includin meniscal extrusion [7, 12-14]. Despite th drawbacks of ultrasound mentioned already, we have shown that it is a reliable technique with excellent diagnostic performance in the assessment of meniscal extrusion when compared with MRI as a reference standard. Because ultrasound is widely available an reliable and has excellent diagnostic performance in the assessment of meniscal extrusion, it could be applied not only to the assessment of static displacement of menisci, as in our study, but also to the assessment of dynamic displacement of menisci.

Although it is possible to reproduce axia loading to the knees using MRI [20], doin so is time consuming and requires special loading devices, making for an ungainly procedure, especially compared with the ease of ultrasound. Furthermore, it is also unlikely that such a procedure would reproduce exactly the physiologic axial load to the knees of the standing position.

If dynamic assessment were possible an practical, the displacement that occurs on the change from supine to weight-bearing positions could be quantified. Although our study found ultrasound to be reliable and accurate in the assessment of medial meniscal extrusion with patients in a supine positio only, we assume that further assessment with

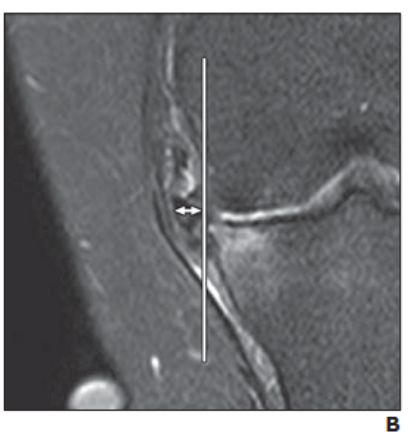

patients in a weight-bearing position would be feasible, because we could frequently depict the external edge of the body of medial menisci in our sample to assess extrusion, when compared with MRI. Therefore, this also should be feasible with the patients standing (weight bearing). So far, however, this use of ultrasound has been little explored [15-17]. Two studies that evaluated meniscal extrusion dynamically with ultrasound, comparing subjects with and without knee osteoarthritis $[16,17]$, showed that not only was meniscal extrusion more frequent in compartments with osteoarthritis but also that it worsened with the change from supine to standing (dynamic displacement). Although this finding hints at some tantalizing possibilities, in fact, the actual significance of dynamic displacement of the meniscus is stil unknown. We do not know whether menisci that exhibit dynamic displacement affect the course of osteoarthritis differently than menisci that are not displaced.

Our study is not the first to assess menis cal extrusion with both ultrasound and MRI In one previous study, non-weight-bearing MRI and ultrasound of the knee performed in the supine, bipedal, and unipedal stances were used to study extrusion of transplanted lateral menisci, comparing them with healthy control group [15]. However, the aim was to assess the feasibility of ultrasound in measuring meniscal displacement before and after applying load to the study knee, but not to directly compare ultrasound and MRI in the assessment of meniscal extrusion.

Of course, our study has its limitations. We did not look for associated abnormalities, such as meniscal tears, in the knee joint. However, by including consecutive subjects with chronic knee pain, we gathered sample with a fair distribution of differen

Fig. 3-59-year-old woman with chronic knee pain. $A$ and $\mathbf{B}$, Ultrasound $(\mathbf{A})$ and MRI $(\mathbf{B})$ assessments of medial meniscal extrusion are shown. Vertical reference line was drawn as tangent to externa edge of medial tibial plateau and was used as reference in both methods. Grade 2 medial menisca is depicted in both images. Note medial osteophytes ( $A$ and $B$ ), as well as marked cartilage thinning in medial tibiofemoral compartment and bone marrow edemalike changes in medial tibial plateau on MRI (B). Ultrasound image (A) was rotated to match orientation (coronal plane) of MRI (B). 


\section{Nogueira-Barbosa et al.}

grades of meniscal extrusion, which allowed the analyses needed for the purpose of this study. Because we used the ultrasound image displaying the maximal medial meniscal extrusion for measurements in each patient, it was possible that the point of extrusion measurement was not exactly the same across all patients. However, because the aim of the current study was to compare extrusion measurements between ultrasound and MRI, we only had to make sure that, for each patient, the point of measurement in both techniques was the same, which we did by placing the skin markers during ultrasound assessment. To enhance reproducibility when comparing ultrasound and MRI measurements of extrusion, we decided to include osteophytes, when present, for measurements in both methods. This might, however, lead to underestimation of meniscal extrusion. Furthermore, only the body of the medial meniscus was assessed for extrusion measurements because the depth reached by ultrasound is insufficient to visualize the entire meniscus, especially the anterior and posterior horns.

Another potential limitation of this study is the fact that we included in our sample only patients eligible for knee MRI, which could have excluded a large number of patients with meniscal extrusion. Finally, because most lateral menisci in our sample did not show extrusion in the assessments performed, statistical analyses would not be possible laterally, and we decided to validate and report only measurements for the medial meniscus. Thus, it is uncertain that our results can also be applied to the lateral menisci.

We conclude that assessment of medial meniscal extrusion with ultrasound is reliable and can be easily used for both quantitative and semiquantitative assessment, showing excellent diagnostic performance for the detection of meniscal extrusion when compared with MRI. Ultrasound is readily available, is less expensive than MRI, and may offer some interesting possibilities for future dynamic evaluation of meniscal extrusion. Taken together, these factors suggest that ultrasound has a good deal of potential for in- creasing our understanding of the causes and consequences of meniscal extrusion and the role extrusion plays in abnormalities of the tibiofemoral compartments of the knee joint.

\section{Reference}

1. Crema MD, Guermazi A, Li L, et al. The association of prevalent medial meniscal pathology with cartilage loss in the medial tibiofemoral compartment over a 2-year period. Osteoarthritis Cartilage 2010; 18:336-343

2. Englund $\mathrm{M}$, Guermazi $\mathrm{A}$, Roemer $\mathrm{FW}$, et al. Meniscal tear in knees without surgery and the development of radiographic osteoarthritis among middle-aged and elderly persons: The Multicenter Osteoarthritis Study. Arthritis Rheum 2009; 60:831-839

3. Englund M, Guermazi A, Roemer FW, et al. Meniscal pathology on MRI increases the risk for both incident and enlarging subchondral bone marrow lesions of the knee: the MOST Study. Ann Rheum Dis 2010; 69:1796-1802

4. Hunter DJ, Zhang YQ, Niu JB, et al. The association of meniscal pathologic changes with cartilage loss in symptomatic knee osteoarthritis. Arthritis Rheum 2006; 54:795-801

5. Lo GH, Hunter DJ, Nevitt M, Lynch J, McAlindon TE, Group OAII. Strong association of MRI meniscal derangement and bone marrow lesions in knee osteoarthritis: data from the osteoarthritis initiative. Osteoarthritis Cartilage 2009; 17:743-747

6. Sharma L, Eckstein F, Song J, et al. Relationship of meniscal damage, meniscal extrusion, mala. lignment, and joint laxity to subsequent cartilage loss in osteoarthritic knees. Arthritis Rheum 2008; 58:1716-1726

7. Crema MD, Roemer FW, Felson DT, et al. Factors associated with meniscal extrusion in knees with or at risk for osteoarthritis: the Multicenter Osteoarthritis study. Radiology 2012; 264:494-503

8. Costa CR, Morrison WB, Carrino JA. Medial meniscus extrusion on knee MRI: is extent associated with severity of degeneration or type of tear? AJR 2004: 183:17-23

9. Lerer DB, Umans HR, Hu MX, Jones MH. The role of meniscal root pathology and radial meniscal tear in medial meniscal extrusion. Skeletal Radiol 2004; 33:569-574

10. Berthiaume MJ, Raynauld JP, Martel-Pelletier J, et al. Meniscal tear and extrusion are strongly associated with progression of symptomatic kne osteoarthritis as assessed by quantitative magnetic resonance imaging. Ann Rheum Dis 2005; 64:556-563

11. Madan-Sharma R, Kloppenburg M, Kornaat PR, et al. Do MRI features at baseline predict radiographic joint space narrowing in the medial compartment of the osteoarthritic knee 2 years later? Skeletal Radiol 2008: 37:805-811

12. Roemer FW, Zhang Y, Niu J, et al. Tibiofemoral joint osteoarthritis: risk factors for MR-depicted fast cartilage loss over a 30 -month period in the multicenter osteoarthritis study. Radiology 2009; 252:772-780

13. Ding C, Martel-Pelletier J, Pelletier JP, et al. Kne meniscal extrusion in a largely non-osteoarthritic cohort: association with greater loss of cartilage volume. Arthritis Res Ther 2007; 9:R21

14. Brody JM, Lin HM, Hulstyn MJ, Tung GA. Lateral meniscus root tear and meniscus extrusion with anterior cruciate ligament tear. Radiology 2006; 239:805-810

15. Verdonk $\mathrm{P}$, Depaepe $Y$, Desmyter $S$, et al. Norma and transplanted lateral knee menisci: evaluation of extrusion using magnetic resonance imaging and ultrasound. Knee Surg Sports Traumatol Ar. throsc 2004; 12:411-419

16. Kawaguchi K, Enokida M, Otsuki R, Teshima R. Ultrasonographic evaluation of medial radial displacement of the medial meniscus in knee osteoarthritis. Arthritis Rheum 2012; 64:173-180

17. Acebes C, Romero FI, Contreras MA, Mahillo I, Herrero-Beaumont G. Dynamic ultrasound assessment of medial meniscal subluxation in kne osteoarthritis. Rheumatology (Oxford) 2013; 52:1443-1447

18. Ko CH, Chan KK, Peng HL. Sonographic imaging of meniscal subluxation in patients with radiographic knee osteoarthritis. J Formos Med Asso 2007; 106:700-707

19. Wareluk P, Szopinski KT. Value of modern sonography in the assessment of meniscal lesions. Eur J Radiol 2012: 81:2366-2369

20. Stehling C, Souza RB, Hellio Le Graverand MP, et al. Loading of the knee during 3.0T MRI is associated with significantly increased medial meniscus extrusion in mild and moderate osteo arthritis. Eur J Radiol 2012; 81:1839-1845 
ANEXO F - Trabalho premiado no congresso internacional RSNA 2012

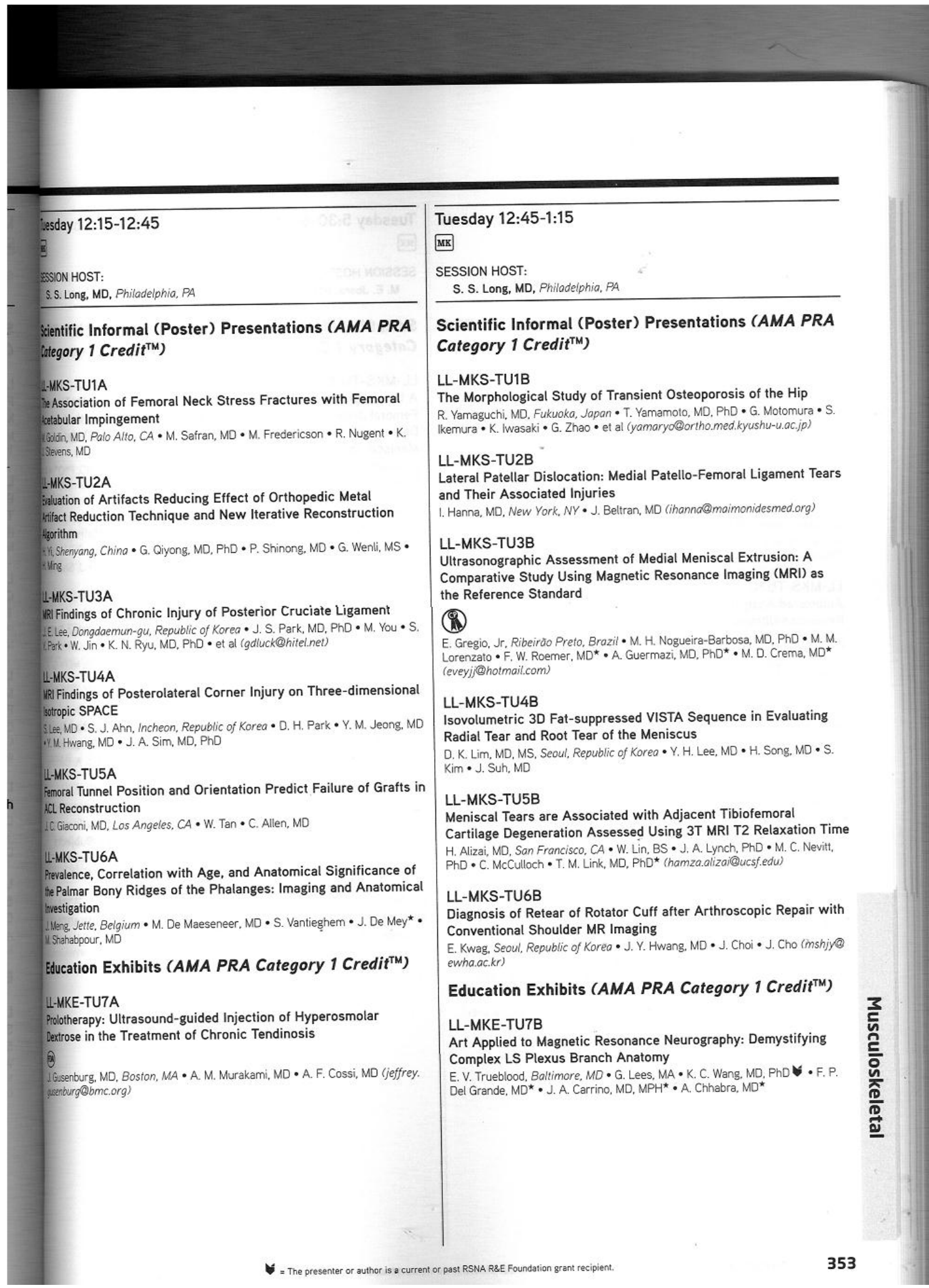




\section{ANEXO G - Trabalho publicado nos anais o RSNA 2012}

Contact: Michel D. Crema

INSTITUTION: Boston University School of Medicine

E-Mail: michelcrema@gmail.com Quantitative Imaging Center Dept of Radiology

Primary Category: Musculoskeletal

Secondary Category: Knee Disorders

Tue Nov 272012 12:45PM - 1:15PM ROOM Lakeside Learning Center

3B) Ultrasonographic Assessment of Medial Meniscal Extrusion: A Comparative Study Using Magnetic Resonance Imaging (MRI) as the Reference Standard

E Gregio-Junior; M H Nogueira-Barbosa, MD, PhD; M M Lorenzato; F W Roemer, MD; A Guermazi, MD, PhD; M Crema, MD, Boston, MA (eveyjj@hotmail.com)

PURPOSE

MRI is commonly used and widely accepted for semiquantitative and quantitative assessments of meniscal extrusion (ME). Ultrasound (US) is widely available and can be applied for the assessment of ME. The aim of this study was to validate the semiquantitative and quantitative assessments of medial ME in US, using MRI measurements as the reference standard.

\section{METHOD AND MATERIALS}

80 consecutive patients referred for knee MRI were also evaluated by US on the same day. US, using a $12-5$ $\mathrm{MHz}$ linear probe, and $1.5 \mathrm{~T}$ routine MRI were performed with patients in a supine position. US was performed at the medial aspect of the knee in the longitudinal axis. Two skin markers were placed in the medial aspect of the knee where ME was assessed. On MRI, the coronal T2w sequence was used to evaluate medial ME, using the slice displaying both skin markers placed during US. For both methods, the edge of the medial tibial plateau was used as the reference for ME measurement. ME was assessed separately by 2 radiologists, and semiquantitatively graded as: $0(<2 \mathrm{~mm}), 1(\geq 2 \mathrm{~mm}$ and $<4 \mathrm{~mm})$, and $2(\geq 4 \mathrm{~mm})$. The agreement comparing ME measurements between US and MRI was assessed using weighted kappa ( $\mathrm{K})$ statistics. Also, intraclass correlation coefficients (ICC) were used to assess agreement using absolute values of measurements (quantitative assessment). Inter-reader reliability for US and MRI ME grades was assessed using $\mathrm{K}$ statistics. The diagnostic performance of US in the detection of ME was evaluated using MRI data as the reference.

\section{RESULTS}

When comparing ME grades between US and MRI, the agreement was moderate for reader $1(\kappa=0.57)$ and substantial for reader $2(\mathrm{k}=0.64)$. When comparing ME absolute values between US and MRI, a substantial agreement was found for both readers (ICC of 0.73 and 0.70 respectively). Inter-reader agreement was substantial for MRI measurements $(\mathrm{K}=0.7)$ and almost perfect for US measurements $(\mathrm{K}=0.98)$. For both readers, US showed excellent sensitivity ( $95 \%$ and $96 \%)$ and good specificity $(82 \%$ and $70 \%)$ in the detection of medial ME.

\section{CONCLUSION}

US is a reliable technique in both semiquantitative and quantitative assessments of $\mathrm{ME}$, showing excellent diagnostic performance in the detection of ME when compared to MRI.

\section{CLINICAL RELEVANCE/APPLICATION}

US is a powerful tool in the assessment of ME, as it may allow for dynamic evaluation from supine to weightbearing positions, which would help the understanding of causes and consequences of ME.

FIGURE (OPTIONAL)

** no data entered ** 
ANEXO H - Trabalho publicado nos anais do ECR 2013

$\mathrm{EPOS}^{\mathrm{TM}}$

Electronic Presentation Online System

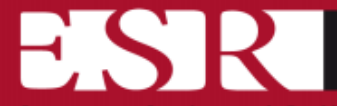

1

Ultrasonographic assessment of medial meniscal extrusion: a validation study using magnetic resonance imaging (MRI) as the reference standard.

\author{
Poster No.: $\quad$ C-1903 \\ Congress: $\quad$ ECR 2013 \\ Type: Scientific Exhibit \\ Authors: $\quad$ M. H. Nogueira-Barbosa ${ }^{1}$, E. Gregio-Junior ${ }^{1}$, M. M. Lozenzato ${ }^{1}$, F. \\ W. Roemer ${ }^{2}$, A. Guermazi ${ }^{3}$, M. D. Crema ${ }^{4} ;{ }^{1}$ Ribeirão Preto, SP/ \\ $\mathrm{BR},{ }^{2}$ Augsburg/DE, ${ }^{3}$ Boston, MA/US, ${ }^{4}$ Brasília, DF/BR \\ Keywords: Image verification, Comparative studies, Ultrasound, MR, \\ Musculoskeletal joint, Extremities \\ DOI: $\quad 10.1594 /$ ecr2013/C-1903
}

Any information contained in this pdf file is automatically generated from digital material submitted to EPOS by third parties in the form of scientific presentations. References to any names, marks, products, or services of third parties or hypertext links to thirdparty sites or information are provided solely as a convenience to you and do not in any way constitute or imply ECR's endorsement, sponsorship or recommendation of the third party, information, product or service. ECR is not responsible for the content of these pages and does not make any representations regarding the content or accuracy of material in this file.

As per copyright regulations, any unauthorised use of the material or parts thereof as well as commercial reproduction or multiple distribution by any traditional or electronically based reproduction/publication method ist strictly prohibited.

You agree to defend, indemnify, and hold ECR harmless from and against any and all claims, damages, costs, and expenses, including attorneys' fees, arising from or related to your use of these pages.

Please note: Links to movies, ppt slideshows and any other multimedia files are not available in the pdf version of presentations.

www.myESR.org

Page 1 of 10 
ANEXO I - Dados brutos dos voluntários
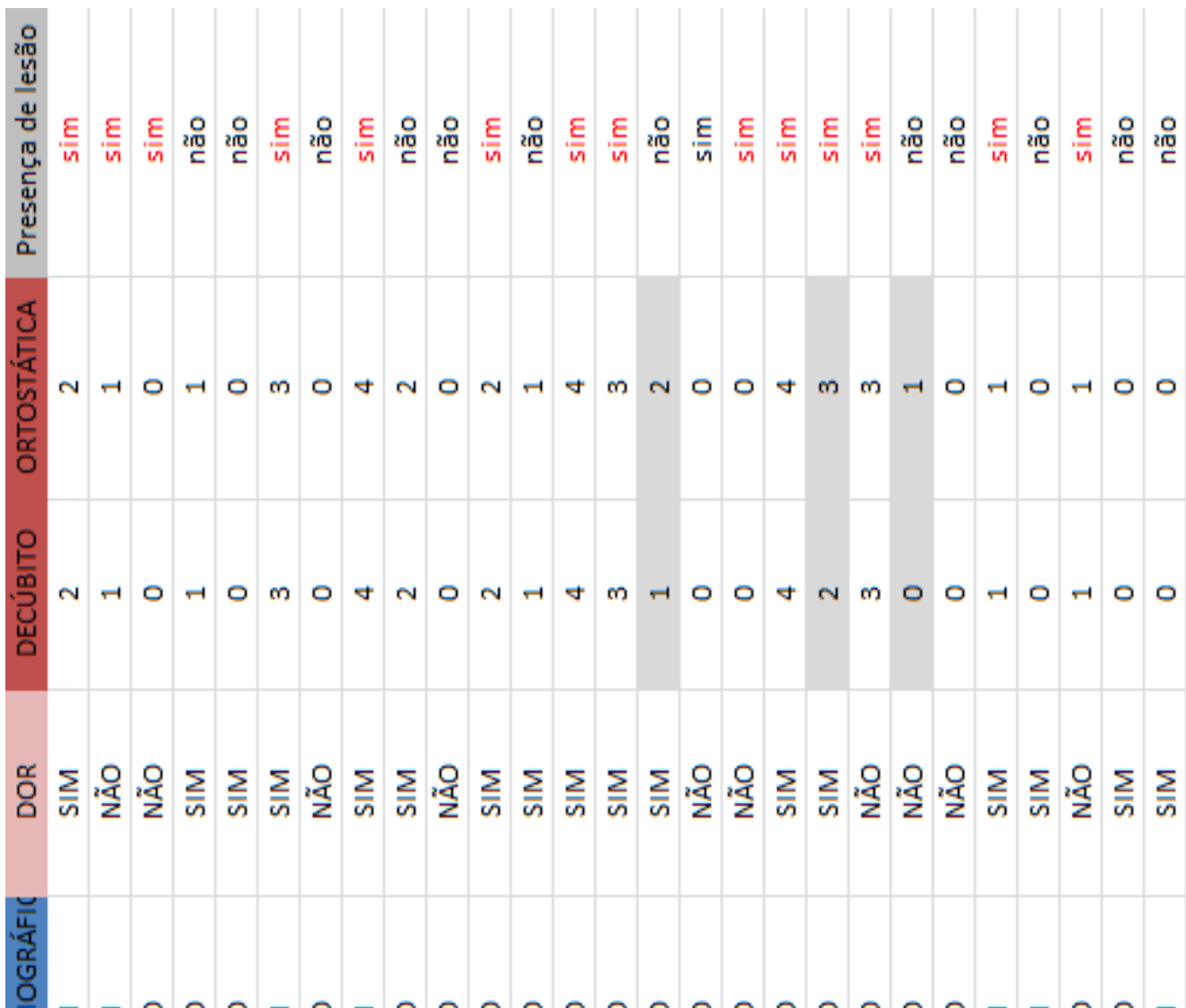

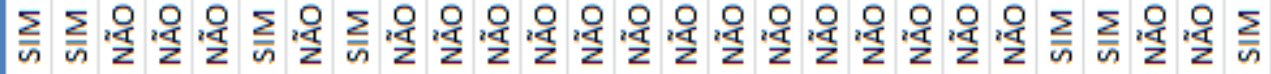

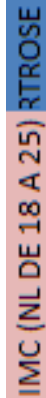

毫守先

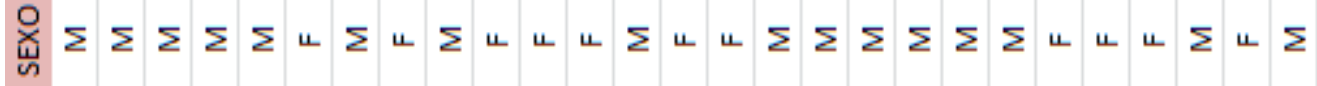

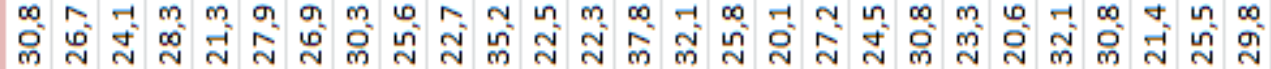

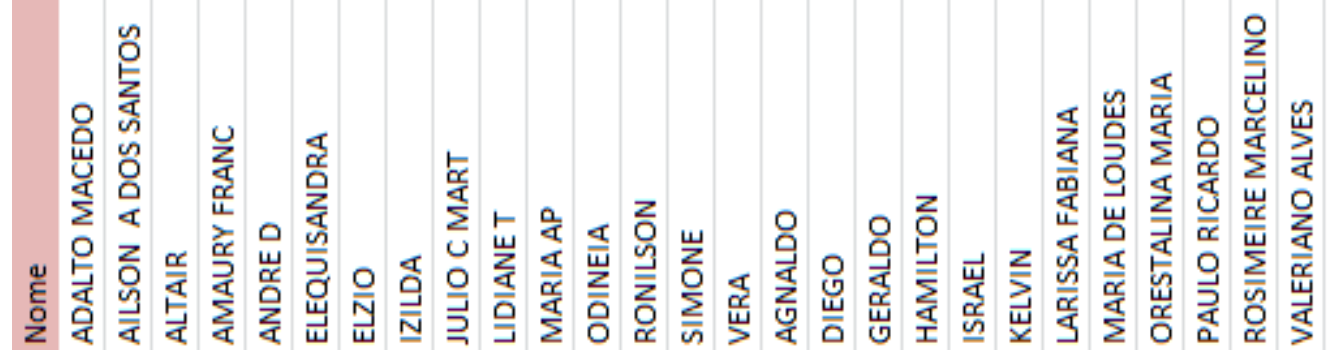




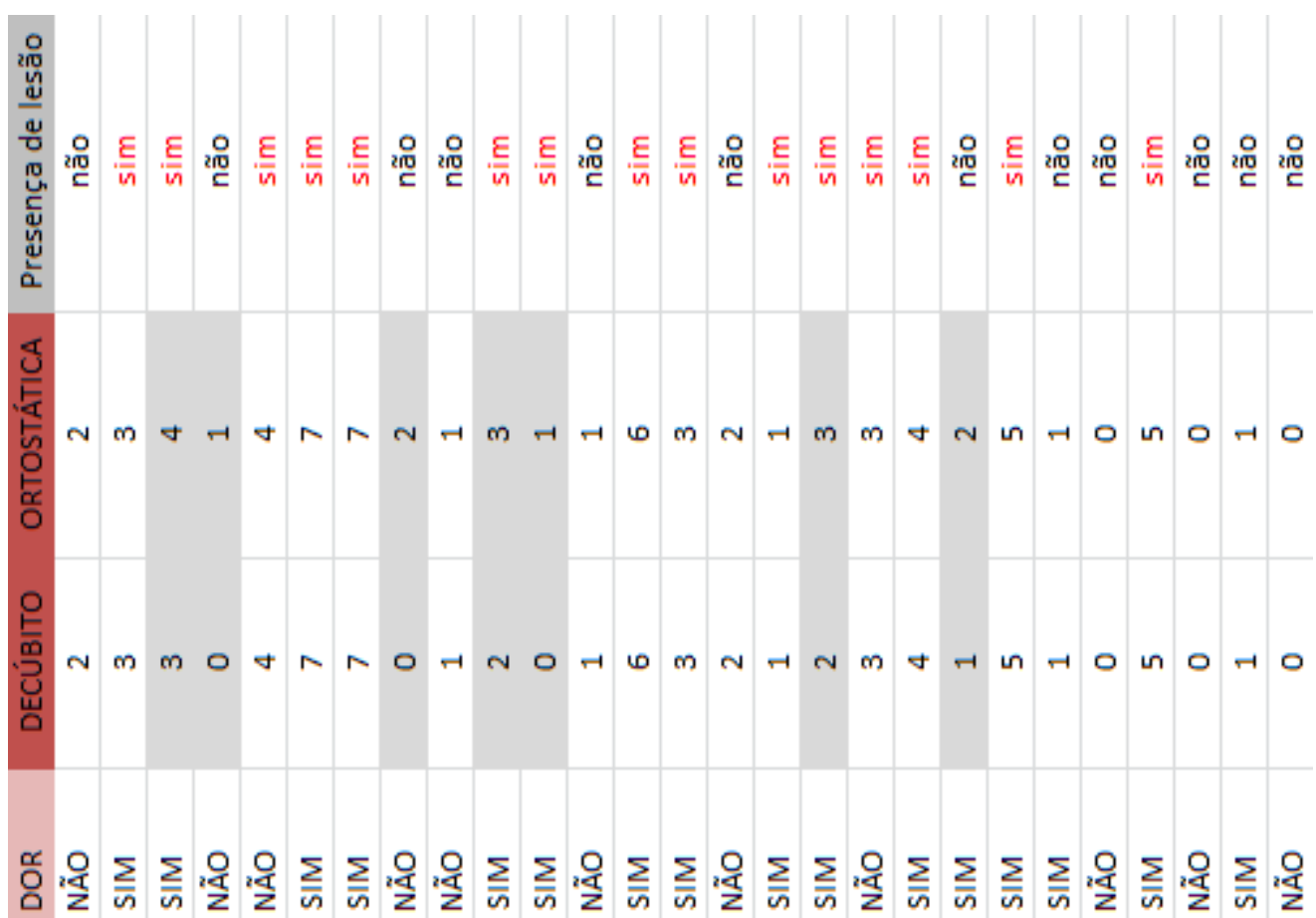

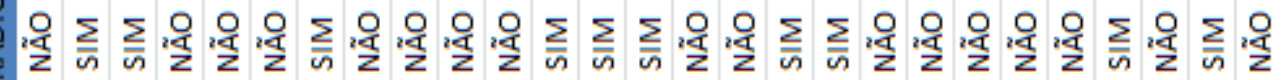

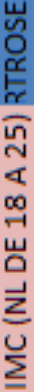

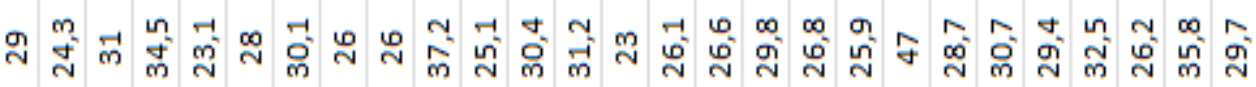

$\underline{\underline{s}}$

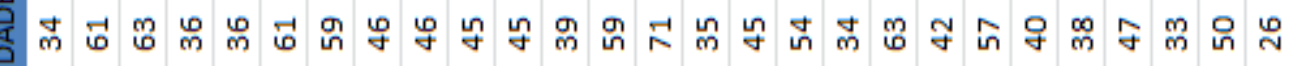

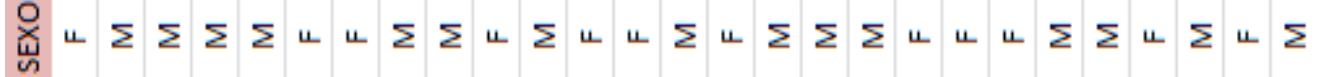

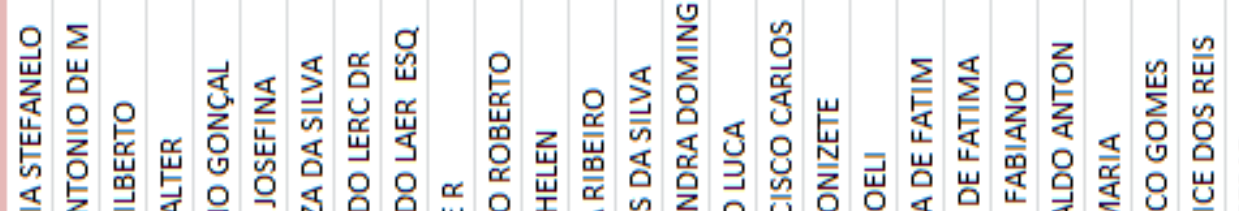

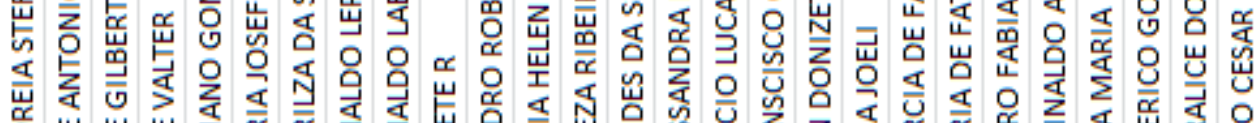

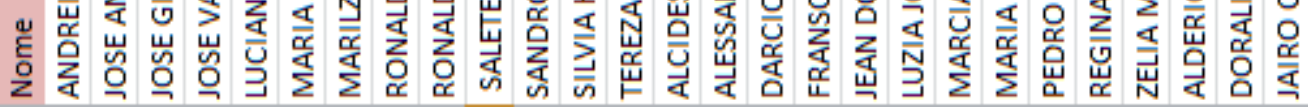




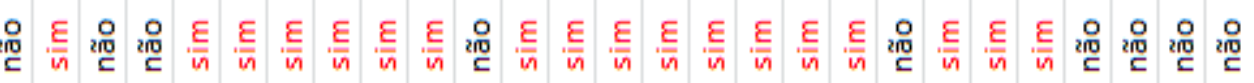

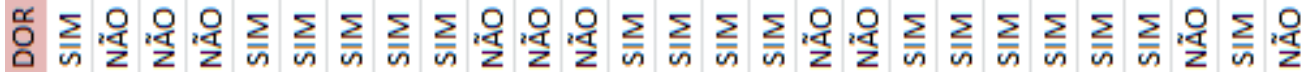

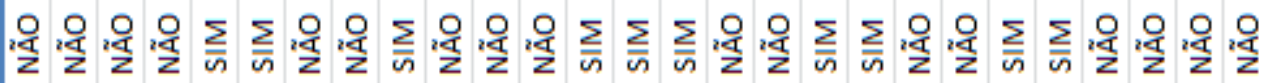

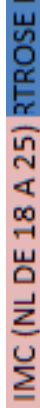

ఇ

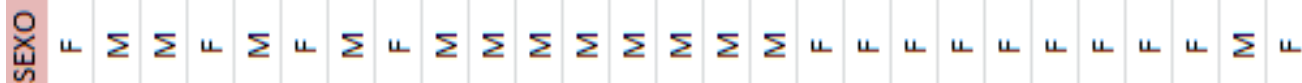

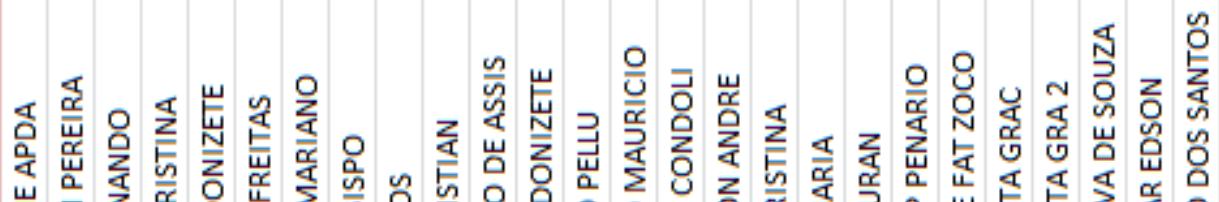

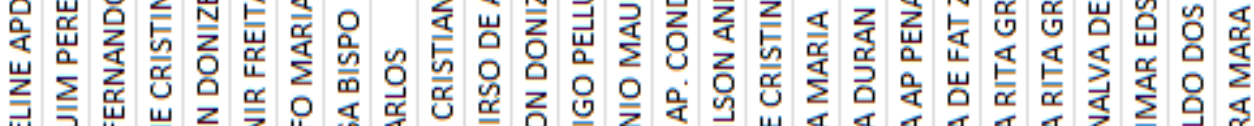

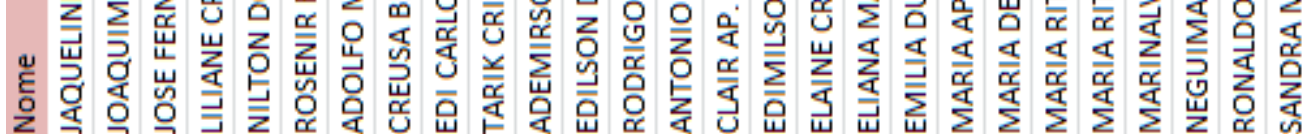



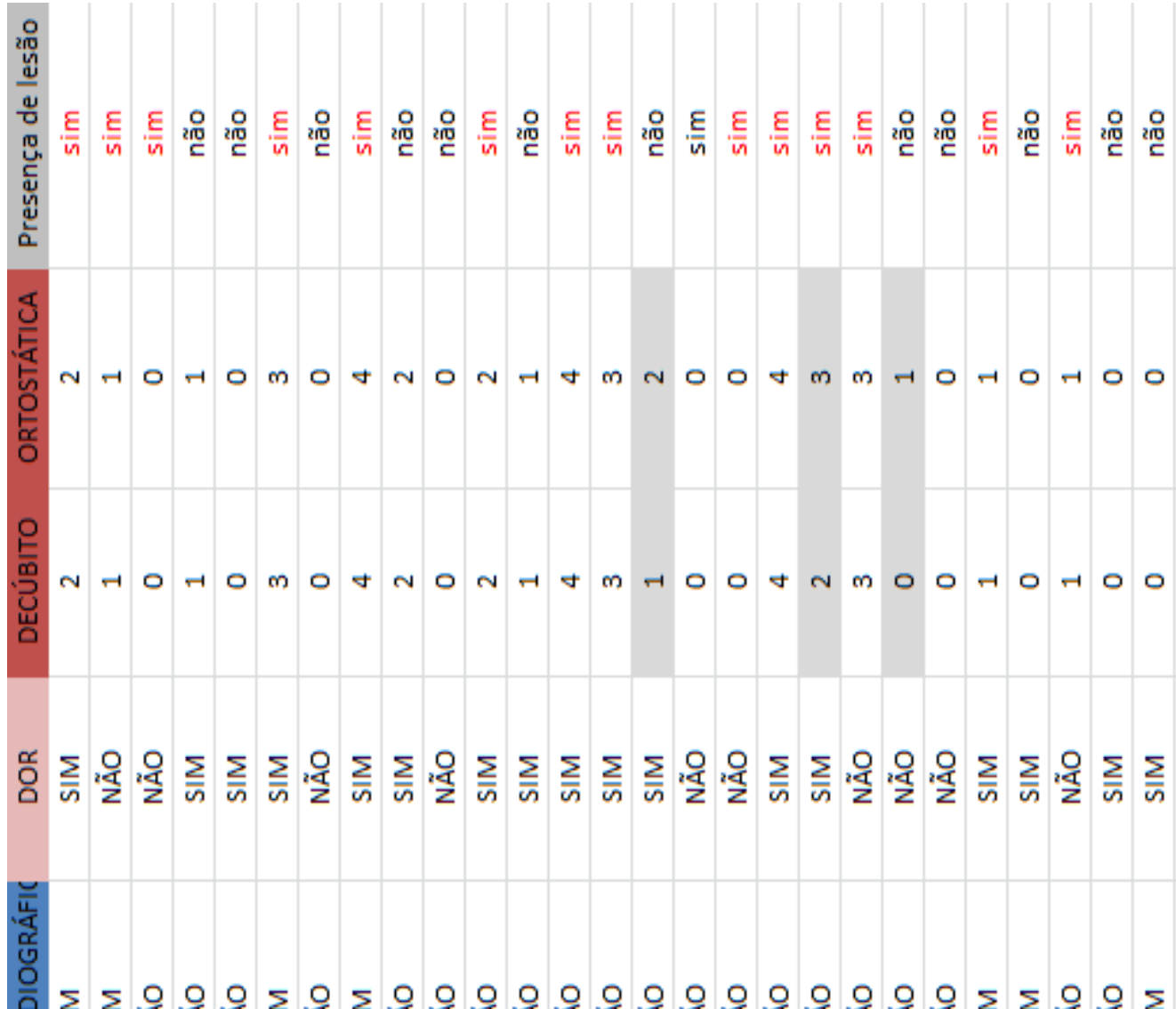

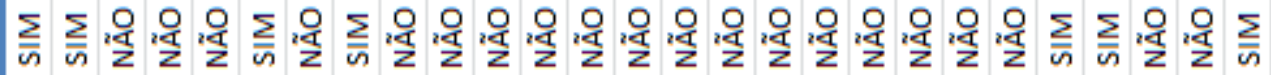

点

实守先

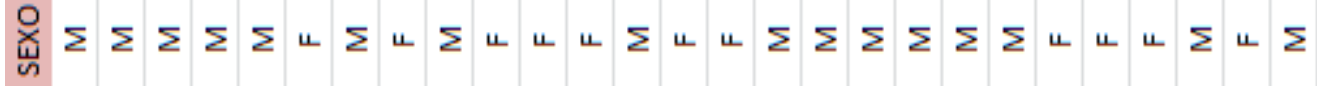

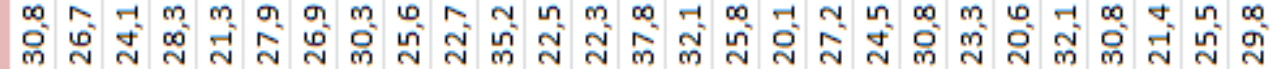

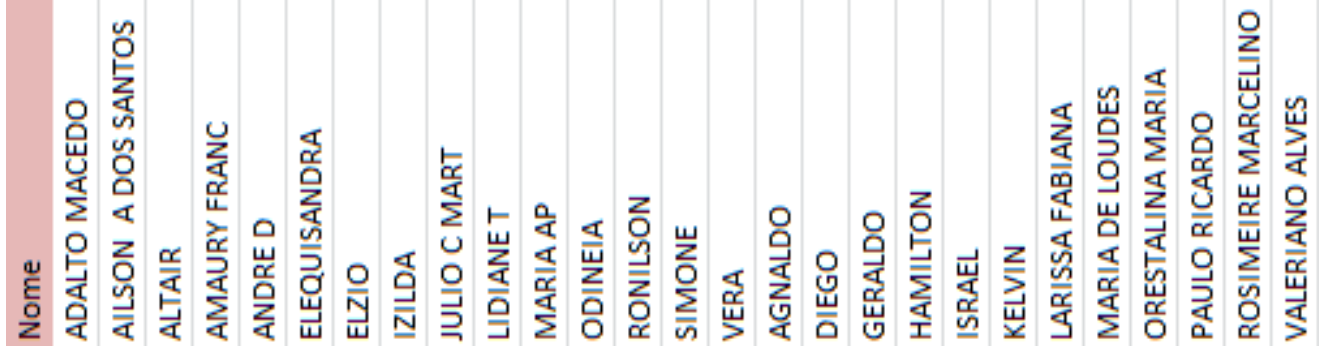




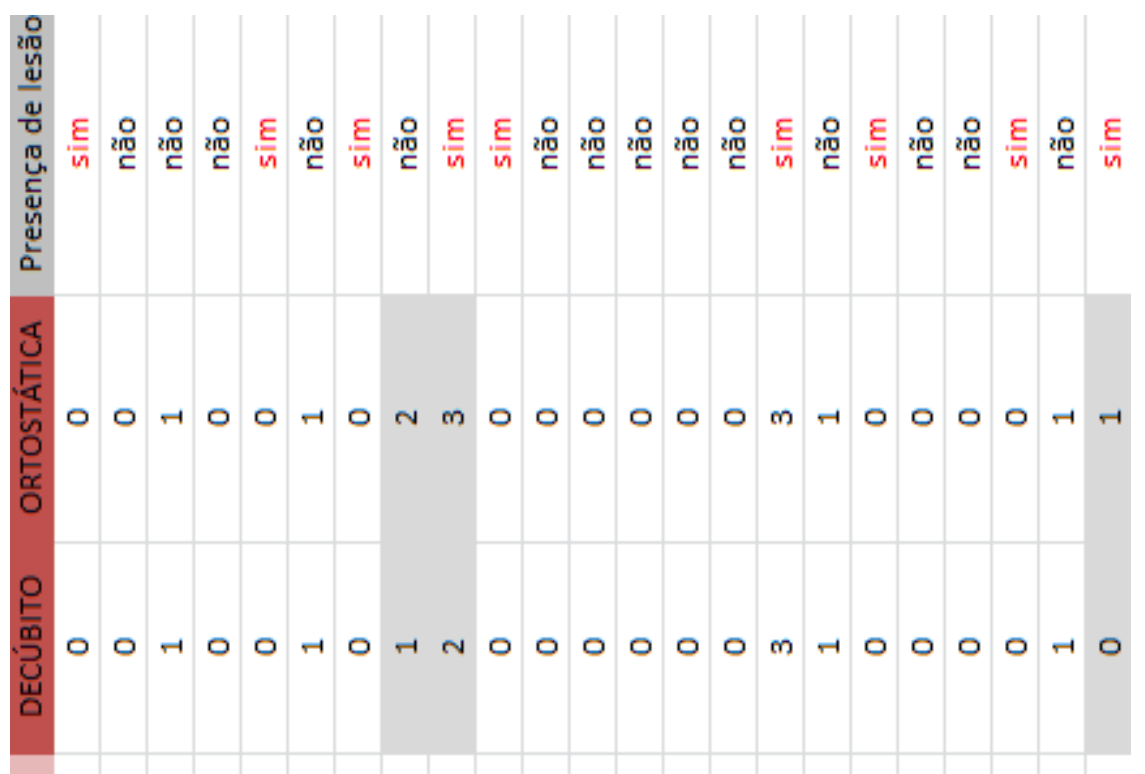

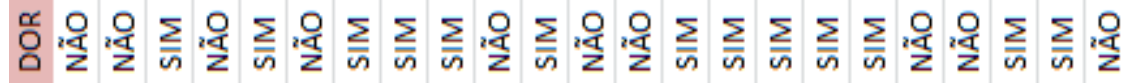

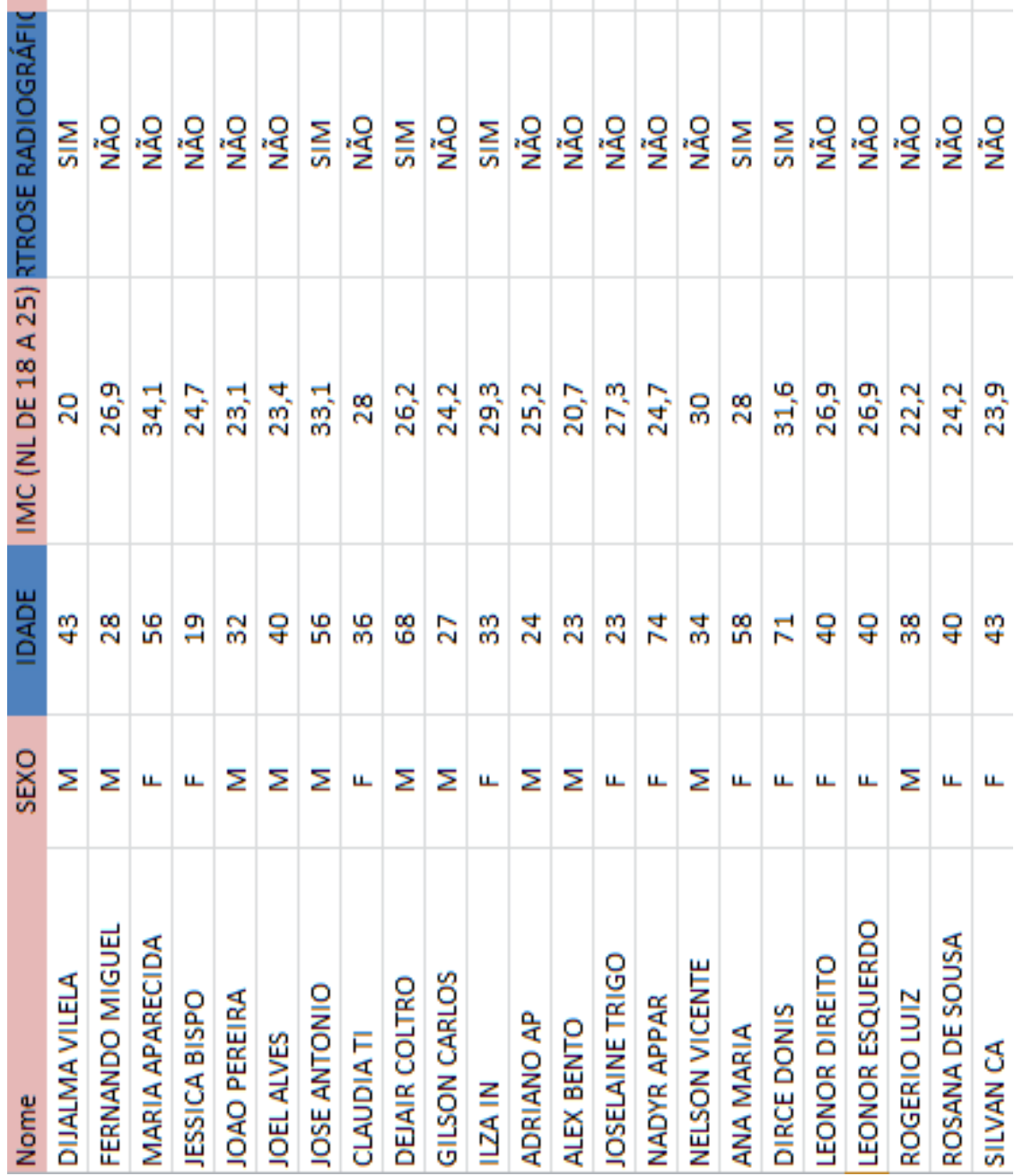

